\title{
An extensive and systematic literature review for hybrid flowshop scheduling problems
}

\author{
Murat Çolak $^{\mathbf{a}^{*}}$ and Gülşen Aydın Keskin ${ }^{\mathrm{b}}$
}

${ }^{a}$ Kocaeli University, Department of Industrial Engineering, 41380, Kocaeli, Turkey

${ }^{b}$ Balikesir University, Department of Industrial Engineering, 10145, Balikesir, Turkey

\section{CH R O N I C L E A B S T RACT}

\begin{tabular}{l}
\hline Article history: \\
Received September 142021 \\
Received in Revised Format \\
November 12021 \\
Accepted December 132021 \\
Available online \\
December, 132021 \\
\hline Keywords: \\
Hybrid flowshop \\
Production scheduling \\
Literature review \\
PRISMA
\end{tabular}

\section{Introduction}

Scheduling is a decision-making process which is utilized in many manufacturing and service industries and it deals with allocation of resources to tasks and aims to optimize one or more objectives (Pinedo, 2008). There are different scheduling environments such as single machine, parallel machine, flow shop, job shop, and open shop in the literature and these types of scheduling problems are handled with different constraints and objectives. Flowshop scheduling problems were introduced by Johnson (1954). A set of jobs are processed unidirectionally in a series of stages (stage 1, stage 2,..,stage k) where each stage has a single machine in the classical flowshop scheduling problems. Hybrid flowshop scheduling problem (HFSP) is a combination of parallel machine and flowshop scheduling environments and there are $\mathrm{k}$ stages that at least one of these stages has parallel machines (identical, uniform or unrelated) instead of $\mathrm{m}$ machine in series as seen in the classical flowshops. Each job has to be processed unidirectionally (stage 1, stage 2, and so on) and by only one and any machine at each stage. In this type of scheduling problems, it is aimed to assign jobs to parallel machines at each stage and to sequence jobs assigned to each machine. Hybrid flowshops are also known as flexible flowshop and multiprocessor flowshop in the literature (Pinedo, 2008; Johnson, 1954; Ribas et al., 2010).

* Corresponding author

E-mail: colak.murat@kocaeli.edu.tr (M. Çolak)

2022 Growing Science Ltd. doi: $10.5267 /$ j.ijiec.2021.12.001 
While the most common application area of HFS is manufacturing, there are different applications of HFSP in the literature. These applications can be sampled as scheduling in hospital sterilization plant (Rossi et al., 2013), container handling systems scheduling (Assadipour et al., 2014; Li et al., 2016; Qin et al., 2020), operating theater scheduling (Bing-hai et al., 2016), integrated surgery scheduling (Hachicha and Mansour, 2018), outpatient scheduling (Chabouh et al., 2018), patient assignment scheduling ( $\mathrm{Li}$ et al., 2019a), task scheduling in cloud computing system ( $\mathrm{Li}$ and Han, 2020), train scheduling (Boroun et al., 2020), carrier aircraft sortie scheduling (Liu et al., 2020a), land-use crop planning (Guan et al., 2020) and sugarcane field preparation (Worasan et al., 2020). It is obvious that there are many application areas for hybrid flowshops such as healthcare management, transportation, cloud computing and agriculture except manufacturing.

When the literature is examined, it is seen that there are several review papers related to HFSP which handle this problem from different points of views in the last decade. These studies have been briefly summarized in the following. Ribas et al. (2010) focused on the papers published from 1995 to 2010 in their review study and classified these papers according to machine characteristics (identical, uniform or unrelated), job constraints, objective functions and solution methods. Ruiz and Vazquez-Rodriguez (2010) analyzed more than 200 papers published before the year 2010 and classified these papers by dividing three categories in terms of solution algorithms as exact algorithms, heuristics and metaheuristics. Morais et al. (2013) determined 72 papers published between 1991 and 2012 and analyzed these papers according to their solution approaches, objective functions and additional constraints by classifying in terms of setup times (sequence-dependent or sequence-independent). Neufeld et al. (2016) determined 112 papers which are related to flowshop group scheduling (including HFS papers) and published from 1976 to 2015 and analyzed these papers by categorizing them according to setup time, objective function and solution method. Gonzalez-Neira et al. (2017) realized a review study by identifying 100 papers which are related to classical flowshop and hybrid flowshop scheduling under uncertainties and published in 2001-2016 year interval. They classified and analyzed these papers according to some characteristics such as uncertain parameters, optimization method and objective function and aimed to present future research opportunities. Hwang and Lin (2018) presented a review of more than 37 papers related to production models developed for two-stage hybrid flowshops with dedicated machines and analyzed these papers in terms of solution approach and computational complexity.

In this study, a more systematic, extensive, and updated review study compared to existing review papers in the literature has been realized for HFSP. It has been aimed to examine existing literature, to present recent developments and to determine research gaps and thus to provide a beneficial road map to researchers who study related to this problem. For this aim, PRISMA methodology which enables systematic reviews and meta-analyses has been utilized and 172 articles have been determined for literature analysis. We searched five different academic databases such as Scopus, Science Direct, Web of Science, Taylor \& Francis Online and Wiley Online Library with various keywords for a more extensive and stricter review process. We utilized some exclusion criteria to provide a focused review study and handled the papers which are published between 2010 and 2020, related to production scheduling, and including a HFS application (exclude review papers, complexity proofs etc.). It has analyzed the papers published 2010 or later to provide an updated review study and to present recent developments with respect to HFSPs. Moreover, we included the papers which present a mathematical programming model to clarify scheduling problems and aimed to analyze these papers in terms of number of stages, type of parallel machines, characteristics related to HFS, objective functions, solution algorithms, test instances and type of parameters rather than structural and complexity analyses of mathematical models. After implementation of PRISMA methodology, 2406 records have been diminished to 172 articles. The included papers have been analyzed and classified according to many characteristics such as year, country, journal, number of stages, type of parallel machines, constraints, objective functions, solution algorithms, test instances and type of parameters, and the results of meta-analyses have been presented through charts for visual demonstration.

The rest of this paper has been organized as follows. HFSP has been detaily explained in Section 2. In Section 3, the review methodology has been introduced and the papers utilized in meta-analyses have been classified according to solution algorithms. Literature analysis results for included papers have been presented through charts in Section 4 . The paper has been concluded with conclusions and future research suggestions in Section 5.

\section{Hybrid flowshop scheduling problem}

In the hybrid flowshop scheduling problem (HFSP), a set of $\mathrm{n}$ jobs is processed in $\mathrm{k}(\geq 2)$ production stages that at least one of these stages has $M_{k}$ parallel machines (identical, uniform or unrelated) (Ribas et al., 2010). A flowchart for HFS environment has been given in Figure 1. The characteristics for standard form of HFSP have been presented as follows (Ruiz and Vazquez-Rodriguez, 2010):

- At time zero all jobs and machines are available.

- At a given stage parallel machines are identical.

- At a specified time period any machine can process only one job and any job can be processed by only one machine.

- All setup times are negligible.

- Preemption is not allowed.

- There is an unlimited buffer between two successive stages.

- The problem data is deterministic and known in advance. 


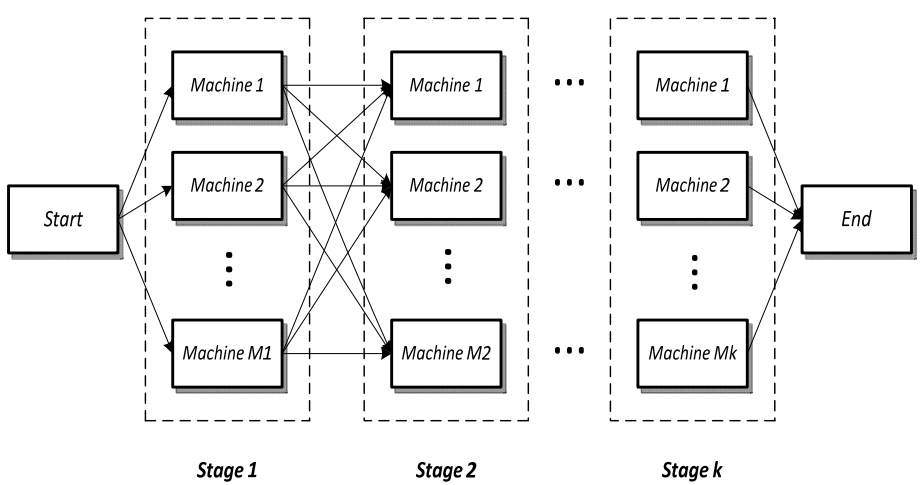

Fig. 1. The hybrid flowshop scheduling environment (Kurdi, 2019)

It is possible to describe HFSP by means of a triplet $\alpha / \beta / \gamma$ notation proposed by Graham et al. (1979). In this notation, while $\alpha$ defines machine environment or shop configuration, $\beta$ indicates the constraints and assumptions of scheduling problems. Finally, $\gamma$ presents the objective functions required to be optimized (Li et al., 2015). There may be three types of parallel machines as identical, uniform and unrelated in the $\alpha$ field of HFSP. These machines are distinguished from each other according to their speeds. Identical machines have the same speeds and therefore each job has the same processing time in these machines. Uniform machines have different speeds and there is a relationship among the processing times of jobs which is inversely proportional to the speed of the machine. Unrelated machines also have different speeds which depend on specific jobs and the processing time of each job is different on different two machines of the same stage (Meng et al., 2019). Besides, it is possible that there is a single machine at one of the stages of the HFS environment.

The second field $\beta$ lists the constraints and assumptions related to HFSP. The constraints considered in different HFSPs have been briefly explained as follows (Pinedo, 2008; Ribas et al., 2010; Ruiz \& Vazquez-Rodriguez, 2010; Karimi et al., 2010; Behnamian et al., 2010; Behnamian \& Zandieh, 2011; Javadian et al., 2012; Defersha \& Chen, 2012; Huang et al., 2015a; Rahmani \& Ramezanian, 2016; Ramezanian et al., 2017; Engin \& Engin, 2018; Feng et al., 2019; Liu et al., 2020b; Ding et al., 2020; Marichelvam et al., 2020; Oztop et al., 2020):

$>$ Release dates $\left(r_{j}, r_{m}\right)$ : It is also known as ready date and it indicates the earliest time that a job can be processed in a machine or stage. It can be valid for machines because of some technical requirements and defines the time that a machine can be utilized.

Setup times (SIST/SDST): Setup times are significant in the manufacturing environment and they are divided into two main parts as sequence-dependent setup times (SDSTs) and sequence-independent setup times (SISTs). The required setup time is dependent on both prior and current jobs in SDSTs and these types of setups are mostly utilized in real manufacturing environments. SISTs are included in the processing times. Setup times are also classified as anticipatory and nonanticipatory. If the setup is anticipatory it can be started before the job is available on the machine, otherwise the setup is defined as non-anticipatory, meaning that the setup can be performed when both the job and machine are available.

> Preemption (prmp): It indicates that it is not necessary to stay a job on a machine from starting to completion.

Precedence (prec): It indicates precedence relationships between jobs, in the HFSPs there is no precedence constraints between jobs but there is between different operations of a job.

> Permutation (prmu): It states that all jobs are processed in the same order in every production stage.

Job families $(\mathrm{fm} l \mathrm{~s})$ : The $\mathrm{n}$ jobs belong to f different job families in the group scheduling problem. There is no setup between jobs of a family, SDST or SIST is required while changing from one family to another.

$>$ Batch processing (scheduling) (batch): It states processing of jobs as batches instead of single processing.

Machine eligibility $\left(M_{j}\right)$ : It is also known as dedicated machines and states that a job can be processed in a predetermined subset of parallel machines in any stage because of technical or physical constraints.

L Limited buffer: It states that the storage area between two consecutive stages is limited and can cause blocking in the upstream machine.

$>$ Blocking (block): It means that the completed job remains on the upstream machine because of zero or limited buffer between two successive stages and blocks this machine.

No-wait $(n w t)$ : It indicates that jobs are not allowed to wait between two consecutive stages due to zero buffer or technological requirements and for two consecutive stages the completion time in the previous stage and the starting time in the next stage are the same for a job. 
$>$ Recirculation $(r c r c)$ : It indicates that a job may revisit any stage several times in other words the jobs are allowed to be processed more than once in a stage.

> Machine breakdown (brkdown): It implies that a machine may not be available because of breakdown as a stochastic event.

$>$ New job arrival: It indicates an unexpected arrival of a new job after the start of the process as a stochastic disruption.

Lot sizing: This constraint indicates the production quantity of lots for each job.

$>$ Lot streaming (lotstream): It is a technique that enables splitting a job into sublots which are equal-sized or variable-sized, to reduce makespan by transferring these sublots to a number of machines and it is also known as job splitting.

Sizeij: It states that the number of machines required to process a job at a stage. Besides, it is possible to indicate that a task of a job has to be processed sizeij of parallel machines at a stage.

$>$ Stage skipping (skip): It is a feature that gives flexibility to scheduling problems and states the probability of not being processed for a job at some stages.

$>$ Limited waiting times (limwait): It indicates that the waiting time between two successive stages cannot be greater than a predetermined upper bound for all jobs.

$>$ Transportation (removal) times $\left(t_{j}\right)$ : It defines the time that is required for transportation of jobs between two consecutive stages.

$>$ Time lag: It indicates the duration between completion and starting times of a job in the consecutive two stages.

$>$ Time/due window: It is a time interval that indicates starting and completion times of a job and earliness/tardiness penalties occur if the job is completed outside of this interval.

$>$ Learning/forgetting effect (LE/FE): LE indicates a constraint that depends on age and skill of workers and affects the processing time. FE states to consider the forgetting possibility of previous jobs when performing different jobs.

$>$ Preventive maintenance $(P M)$ : It is a timely and periodic activity which aims to prevent unexpected failures of machines and keep them in good condition. Besides, it means that machines are not available for a time period.

$>$ TOU electricity prices (TOU): It indicates the fluctuation of electricity prices over time and they may change from hour to hour in a day.

$>$ Turn on/off strategy (on/off): It is a strategy that enables us to reduce electricity costs in a scheduling environment.

$>$ Variable speed levels: It indicates that the speed selection is possible for different jobs due to variable speed levels of machines and therefore the processing time and energy consumption may change.

On the other hand, cyclic scheduling, assembly scheduling, proportionate scheduling and distributed scheduling are the concepts studied in the HFS field as specific scheduling environments. A brief definitions of these concepts have been given as follows (Sawik, 2012; Shiau \& Huang, 2012; Nikzad et al., 2015; Li et al., 2020a):

* Cyclic scheduling: It is aimed to create a minimal part set (MPS) to schedule repetitively in this scheduling approach. While the cycle of parts in MPS is not stated as a precedence, it is obtained with the optimal schedule for all parts.

* Proportionate scheduling: It is a combination of proportionate flowshop scheduling and identical parallel machine scheduling under HFS environment. That is, it is indicated that each job has the same processing times in different stages.

* Assembly scheduling: It indicates that there is an assembly stage for assembly operations of components to produce the final product in the scheduling environment.

* Distributed scheduling: It is an extension of HFSP and involves three sub-problems such as selecting a factory for each job, allocating a machine for each job at each stage and determination of job sequence for each job allocated to any parallel machine.

The third field $\gamma$ demonstrates the objective functions aimed to be optimized and this field can include single or multi objective. There are two approaches in the literature to solve multi-objective optimization problems as priori and posteriori. In the a priori approach, appropriate weights are assigned to each objective and the multi-objective scheduling problem is converted to a single-objective problem. It is possible to obtain only one optimal solution by using this approach each time. In the posteriori approach, a set of non-dominated (Pareto optimal) solutions are obtained with the solution of a multi-objective problem each time (Tang et al., 2016). The definitions of significant concepts in the objective functions of HFSPs are as seen in the following.

- $C_{j}$ : Completion time of job $\mathrm{j}$ in the last stage

- $F_{j}$ : Flow time $F_{j}=C_{j}-r_{j}$ 
- $L_{j}$ : Lateness $L_{j}=C_{j}-d_{j}$

- $T_{j}$ : Tardiness $T_{j}=\max \left(C_{j}-d_{j}, 0\right)$

- $E_{j}$ : Earliness $E_{j}=\max \left(d_{j}-C_{j}, 0\right)$

- $U_{j}$ : if job $\mathrm{j}$ is tardy 1 , otherwise 0

The objective functions that can be utilized for HFSPs have been given in Table 1.

Table 1

Objective functions for HFSPs

\begin{tabular}{cccc}
\hline Description & Explanation & Description & Explanation \\
\hline $\max C_{j}$ & Maximum completion time $\left(C_{\max }\right)$ & $\max E_{j}$ & Maximum earliness $\left(E_{\max }\right)$ \\
$\sum C_{j}$ & Total completion time $(T C T)$ & $\sum E_{j}$ & Total earliness $(T E)$ \\
$\sum C_{j} / n$ & Mean completion time $(M C T)$ & $\sum E_{j} / n$ & Mean earliness $(M E)$ \\
$\sum w_{j} C_{j}$ & Total weighted completion time $(T W C T)$ & $\sum w_{j} E_{j}$ & Total weighted earliness $(T W E)$ \\
$\max F_{j}$ & Maximum flow time $\left(F_{\max }\right)$ & $\sum U_{j}$ & Number of tardy jobs $(N T J)$ \\
$\sum F_{j}$ & Total flow time $(T F T)$ & $\sum W_{j}$ & Sum of waiting times $(S W T)$ \\
$\sum F_{j} / n$ & Mean flow time $(M F T)$ & $\sum W_{j} / n$ & Mean waiting time $(M W T)$ \\
$\sum w_{j} F_{j}$ & Total weighted flow time $(T W F T)$ & $T M I T$ & Total machine idle time \\
$\max L_{j}$ & Maximum lateness $\left(L_{\max }\right)$ & $A S T$ & Average sojourn time \\
$\sum L_{j}$ & Total lateness $(T L)$ & $T E C$ & Total energy (electricity) consumption \\
$\sum L_{j} / n$ & Mean lateness $(M L)$ & $T E C C$ & Total energy (electricity) consumption cost \\
$\sum w_{j} L_{j}$ & Total weighted lateness $(T W L)$ & Setup cost \\
$\max T_{j}$ & Maximum tardiness $\left(T_{\max }\right)$ & $P C$ & Production cost \\
$\sum T_{j}$ & Total tardiness $(T T)$ & $W C$ & Worker cost \\
$\sum T_{j} / n$ & Mean tardiness $(M T)$ & $I H C$ & Inventory holding cost \\
$\sum w_{j} T_{j}$ & Total weighted tardiness $(T W T)$ & $O t h r$ & Other specific objectives \\
\hline
\end{tabular}

\section{A Systematic Literature Review for HFSPs}

HFS is a well-known research area and there are many studies with different constraints, objective functions, and solution algorithms related to this subject in the literature. In this section, a systematic review for HFSPs has been realized through PRISMA methodology and studies have been classified according to solution algorithms. The review methodology has been detailed in sub-section 3.1. Afterwards, the included articles have been collected under 8 main topics as exact, heuristic, metaheuristic, exact and heuristic, exact and metaheuristic, heuristic and metaheuristic, exact, heuristic, and metaheuristic and the other methods according to solution algorithms in sub-section 3.2.

\subsection{Review Methodology}

We conducted our review process according to the PRISMA methodology proposed by (Moher et al., 2009a-b) in order to present a systematic review and meta-analyses with respect to HFS. This methodology enables to reveal a detailed and focused review study in a specific research area by using several exclusion criteria and to present some statistical and mathematical results for the examined research subject. Identification, screening, eligibility and inclusion are the four steps of the PRISMA methodology and it is possible to realize an effective review study by following these steps respectively (Kaya et al., 2019). The flowchart for our review process including the steps of PRISMA methodology has been presented in Fig. 2.

\subsubsection{Identification}

In this sub-section, various academic databases such as Scopus, Science Direct, Web of Science, Taylor \& Francis Online and Wiley Online Library have been searched by using various keywords. These keywords can be ranked as hybrid flow shop scheduling (HFSS), hybrid flowshop scheduling (HFS), flexible flow shop scheduling (FFSS), flexible flowshop scheduling (FFS), multiprocessor flow shop scheduling (MPFSS), and multiprocessor flowshop scheduling (MPFS). We utilized five academic databases and different versions of keywords in order to make a more detailed search. The results of the identification step have been presented in Table 2 .

Table 2

The searching results based on different keywords

\begin{tabular}{|c|c|c|c|c|c|c|c|c|}
\hline \multirow{2}{*}{ Academic Database } & \multirow{2}{*}{ Searching Area } & \multicolumn{6}{|c|}{ Results According to Keywords } & \multirow{2}{*}{ Tota } \\
\hline & & HFSS & HFS & FFSS & FFS & MPFSS & MPFS & \\
\hline Scopus & Article title, abstract, keywords & 505 & 182 & 147 & 32 & 13 & 2 & 881 \\
\hline Science Direct & Find articles with these terms & 354 & 151 & 137 & 48 & 15 & 8 & 713 \\
\hline Web of Science & All fields & 268 & 125 & 99 & 26 & 8 & 2 & 528 \\
\hline Taylor \& Francis Online & Anywhere & 132 & 46 & 56 & 12 & 2 & 1 & 249 \\
\hline Wiley Online Library & Anywhere & 15 & 6 & 9 & 3 & 1 & 1 & 35 \\
\hline \multicolumn{2}{|c|}{ Total } & 1274 & 510 & 448 & 121 & 39 & 14 & 2406 \\
\hline
\end{tabular}




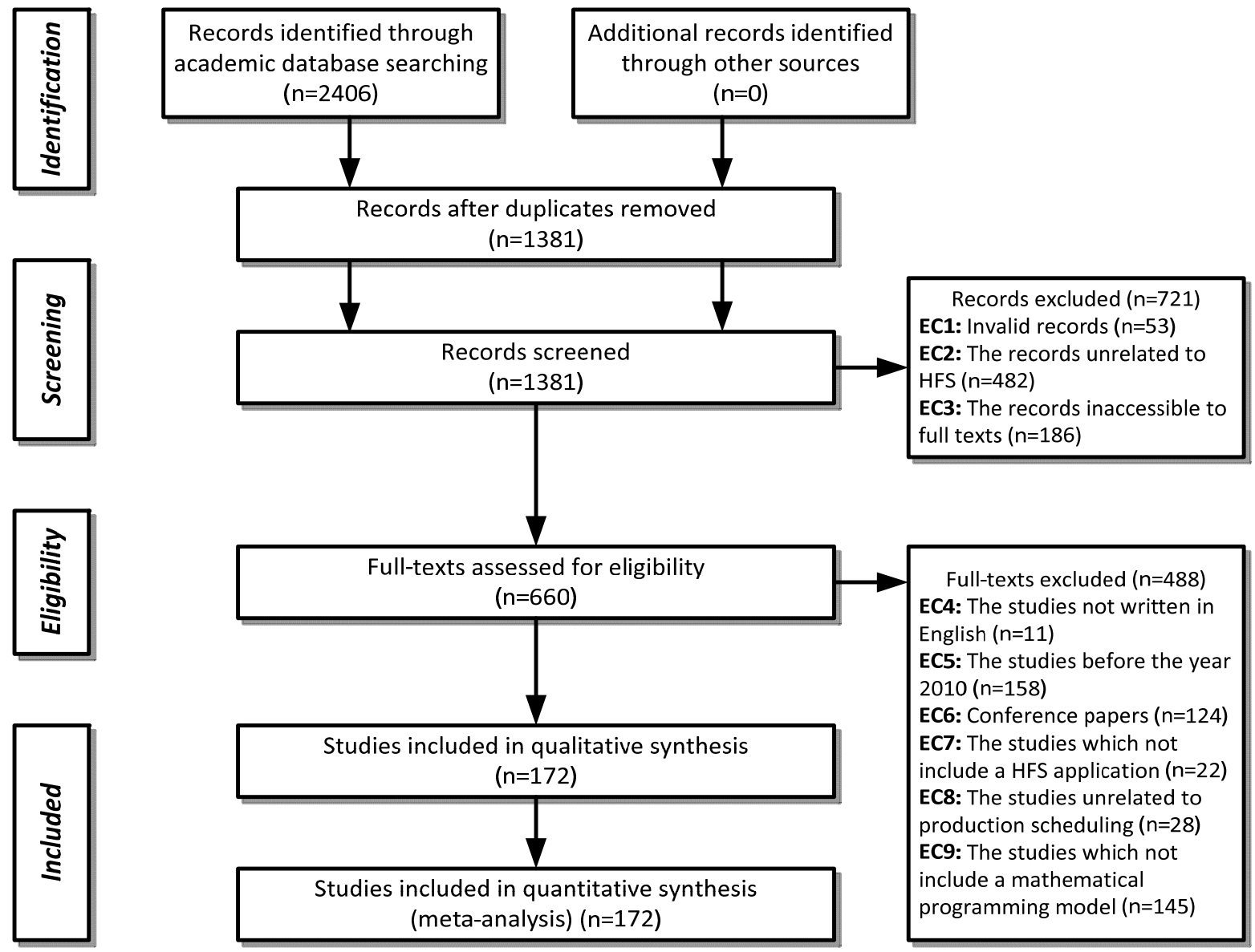

Fig. 2. A flowchart for the review process including steps of PRISMA methodology

\subsubsection{Screening}

In this sub-section, after duplicated papers have been removed in order to handle more reliable statistical results, some papers have been eliminated by using first part exclusion criteria. These three exclusion criteria have been given as follows.

EC1: Invalid records

EC2: The records unrelated to HFS

EC3: The records inaccessible to full texts

\subsubsection{Eligibility}

In this sub-section, remaining papers have been evaluated in terms of eligibility. Some of these papers have also been eliminated with second part exclusion criteria. These six exclusion criteria have been given as follows. By the way, 172 articles have been determined for statistical and mathematical analysis.

EC4: The studies not written in English

EC5: The studies before the year 2010

EC6: Conference papers

EC7: The studies which not include a HFS application

EC8: The studies unrelated to production scheduling

EC9: The studies which not include a mathematical programming model

\subsubsection{Included}

In the last step, 172 articles have been utilized for meta-analyses. Statistical and mathematical analyses have been realized for these articles according to many features such as year, country, journal, number of stages, type of parallel machines, constraints, objective functions, solution methods, test instances and type of parameters. The results of these analyses have been presented via charts to provide a visual demonstration for researchers. 
3.2 Classification of Included HFS Articles based on Solution Approaches

In this sub-section, the included articles determined as a result of methodological review process in Section 3.1 have been classified according to solution methods under 8 main topics as exact, heuristic, metaheuristic, exact and heuristic, exact and metaheuristic, heuristic and metaheuristic, exact, heuristic, and metaheuristic and the other methods with a detailed approach. Besides, a summary classification of included HFS articles according to stage type, type of parallel machines, constraints and characteristics, objective functions, solution algorithms, test instances, and type of parameters has been presented in the chronological order in Table 3.

\subsubsection{Exact Algorithms}

In this sub-section, the articles utilized only an exact algorithm to solve HFSPs have been briefly summarized as follows. Defersha (2011) developed a mathematical model to handle a HFSP including lot streaming and some other constraints and solved this model by CPLEX solver for a small instance to obtain optimal solution. Sawik (2012) presented MIP models for batch, cyclic and batch-cyclic scheduling problems with limited buffer and machine availability constraints under HFS environment. The author solved these models for different instances by using CPLEX solver and compared performance of them. Gicquel et al. (2012) developed a MILP model taking into consideration several constraints for a real-life HFSP arising in the bioprocess industry. They solved this model with an exact method through CPLEX solver adding some cutting planes into the model automatically. Cortes et al. (2012) developed a flexible MIP model with makespan, manufacturing lead time and resource usage objectives for HFSP and proposed augmented $\varepsilon$-constraint method to solve this problem. Wang et al. (2014a) proposed a MILP model for HFSP with job splitting in the solar cell industry and obtained optimum solution for this model through CPLEX solver. Wang et al. (2015) proposed a B\&B algorithm including lower and upper bounds and dominance rules to minimize makespan in a two-stage nwt-HFSP. They demonstrated that the proposed algorithm generates solutions up to 20 jobs in an acceptable computational time. Schulz et al. (2020) presented time-indexed and sequencedependent versions of MIP models for providing energy efficiency and delivery reliability simultaneously in hybrid flowshop. $\varepsilon$-constraint method has been utilized to solve the problem.

\subsubsection{Heuristic Algorithms}

In this sub-section, the articles which apply only a heuristic algorithm to solve HFSPs have been briefly summarized in the following. Nishi et al. (2010) suggested a novel LR method with cut generation in order to solve HFSP with the aim of minimizing TWT. Wang and Choi (2012) presented a decomposition based approach (DBA) to solve HFSP under a stochastic machine breakdown environment and showed effectiveness of the proposed DBA according to either GA or SPT algorithms using random generated test problems (RGTPs). Choi and Wang (2012) proposed a DBA integrating SPT and GA to minimize makespan considering stochastic processing times (SPTs) for a HFSP. They showed superiority of this approach with respect to both SPT and GA through RGTPs. Li et al. (2013a) considered a two-stage HFSP with head group constraint and proposed a constructive heuristic $(\mathrm{CH})$ called as $\mathrm{H}^{\prime}$ in order to minimize makespan for this problem. They revealed the superiority of this algorithm compared with three improved heuristics. Fattahi et al. (2013) studied on an assembly HFSP with makespan objective and presented four heuristics based on Johnson algorithm to solve this problem due to NP-hardness. The performance of these algorithms have been evaluated using two lower bounds. Li et al. (2013b) studied on a two-stage HFSP with tail group constraint and proved NP-hard structure of this problem with a mathematical model. Therefore, they suggested a CH named EL algorithm to solve this problem and revealed the superiority of it with respect to several dispatching rules. Wang and Choi (2014) handled HFSP with SPTs and presented a holonic approach integrating good sides of Genetic Algorithm Control (GAC) and Shortest Processing Time Based Contract Net Protocol (SPT-CNP) with makespan objectives. They determined that the proposed method is more effective than both of these algorithms separately. Jiang et al. (2015) divided SCC problem into two subproblems as a PMSP in the last stage and HFSP in the upstream stages. They applied a hybrid HDE-VNDS algorithm for PMSP and heuristic IBLS algorithm for HFSP in the context of two-phase optimization strategy and evaluated performance of this approach using generated instances from real-world data. Lee et al. (2015) performed a combined heuristic algorithm integrating NEH and beam search algorithms to solve two-stage HFSP and they revealed computational efficiency of this algorithm compared with GA and SA metaheuristics as well as a NEH-based heuristic. Cui and Luo (2017) developed a MINLP model for SCC problems and proposed a LR approach to obtain nearoptimal solutions in a reasonable time for this problem by relaxing complex constraints. They also presented a $\mathrm{CH}$ to provide a feasible schedule and evaluated effectiveness of this heuristic. Shim et al. (2017) proposed a heuristic algorithm constructed with dispatching rules and economic lot size model and aimed to minimize total tardiness of jobs for a HFSP by means of this heuristic. Wang et al. (2018) developed a list scheduling heuristic and its improved version as CHs along with a MILP for a two-stage HFSP. They assessed performance of these algorithms compared with existing heuristic algorithms in the literature. Cui et al. (2020) utilized LR method to solve SCC problem which is a special case of HFS and also presented a CH to obtain a feasible schedule. 


\subsubsection{Metaheuristics}

In this sub-section, the articles which utilize just a metaheuristic to obtain a near-optimal solution for HFSPs have been considered. These articles have been classified according to type of metaheuristics as single (only one metaheuristic) and hybrid metaheuristic (metaheuristic + metaheuristic, metaheuristic + heuristic, metaheuristic + another method) and briefly summarized.

Single metaheuristics are commonly utilized in order to solve HFSPs for large instances as seen in the following articles. Karimi et al. (2010) applied multi-phase GA to minimize makespan and TWT objectives under HFS environment considering SDSTs and showed high performance of this algorithm compared with multi-objective GA. Behnamian and Zandieh (2011) applied ICA for HFSP with SDSTs and limited waiting time constraints and as a result of computational experiments they showed that this algorithm generates good solutions and outperforms two algorithms existing in the literature. Solano-Charris et al. (2011) considered a two-stage HFSP to optimize makespan and TCT simultaneously and proposed an ACO algorithm to solve this problem. They showed superiority of this metaheuristic according to two existing heuristic algorithms. Luo et al. (2011) applied GA to solve a two-stage HFSP and evaluated performance of this algorithm comparing its results with manual schedule for real production data obtained from a metalworking company. Niu et al. (2012) applied a method called QIA which integrates immune algorithm and quantum search to solve HFSP with mean flow time objective. Jolai et al. (2013) performed three versions of SA algorithm to solve two-stage nwt-HFSP and compared these algorithms to each other using performance metrics to determine the most effective one. Attar et al. (2013) proposed the BBO algorithm to solve a HFSP with some practical constraints and demonstrated that it is superior to ICA and population-based SA algorithms. Lin et al. (2013) applied an ABC-based algorithm to solve multiprocessor task scheduling problems (MTSP) under HFS environment and evaluated performance of this algorithm compared with other algorithms through well-known BPs. Li et al. (2014a) applied an improved FFO algorithm so as to solve realistic SCC problems effectively and effectiveness of this algorithm has been shown compared with four existing algorithms. Attar et al. (2014) after developing a mathematical model, presented multi-objective PSO (MOPSO) and SPEA-II algorithms to minimize makespan and TWT objectives simultaneously in a HFSP. They determined that MOPSO is superior to SPEA-II in terms of solution quality as a result of numerical analyses. Ghodratnama et al. (2015) formulated a mathematical model for a three-objective HFSP and because of NP-hardness they suggested MOPSO and NSGA-II method to solve this problem. It has been evaluated performance of these algorithms through comparison metrics. Rahmani and Ramezanian (2016) formulated a model to address HFSP with unexpected disruptions rooted from new job arrivals and performed a VNS algorithm to solve this problem in a reasonable computational time owing to NP-hardness. The effectiveness of this algorithm has been demonstrated as a result of comparisons with some other algorithms according to performance measures. Tang et al. (2016) applied an improved version of PSO algorithm to consider energy-aware HFSP with machine breakdown and new job arrivals. They evaluated performance and effectiveness of the proposed algorithm with respect to some other existing algorithms using BPs. Yan et al. (2016) utilized GA in order to solve energy-efficient HFSP minimizing makespan and total energy consumption and showed effectiveness of this method. Pan (2016) studied the SCC problem which is a combination of charge scheduling (HFS) and cast scheduling (PMS). The CCABC algorithm has been proposed so as to solve this problem and the author demonstrated the effectiveness of this algorithm using random generated and real-life instances. Zhang et al. (2017) developed a mathematical model for hybrid flowshop lot streaming problem and proposed modified MBO algorithm to obtain effective solutions in a reasonable time for this problem. They demonstrated that the proposed algorithm is able to solve HFSP better compared to seven algorithms existing in the literature. Kurdi (2019) performed an ACO-based algorithm for MTSP under HFS environment with makespan objective and evaluated performance of this algorithm with respect to other algorithms testing with well-known BPs. Luo et al. (2019) focused on energy-conscious HFSP with new job arrivals and applied parallel GA with a rescheduling strategy to solve this problem. Geng et al. (2020a) studied reentrant HFSP with TOU electricity prices and proposed an improved ALO algorithm to minimize makespan and TECC. The effectiveness of this algorithm with respect to different multi-objective algorithms has been revealed with performance metrics. He et al. (2020) developed a multi-objective mathematical model for rush order insertion rescheduling problem with several constraints and utilized NSGA-III algorithm to obtain good solutions for this problem. The suitability, effectiveness, and applicability of the proposed algorithm have been shown compared with other algorithms and with a real case application (RCA) from the shipbuilding industry. Liu et al. (2020b) developed a novel mathematical model based on the mixed production mode for energy-aware HFSP and showed suitability of this model so as to optimize makespan and TEC simultaneously by means of real case data. Fu et al. (2020) proposed multi-objective ROA to solve green HFSP and demonstrated effectiveness of this algorithm in order to minimize makespan, noise and dust pollution simultaneously according to values of comparison metrics. Cao et al. (2020a) focused on HFSP with unrelated parallel machines and applied an improved GS algorithm to obtain good solutions for this problem. Effectiveness of the proposed algorithm has been shown compared with some existing algorithms and performing for a RCA. Kong et al. (2020) presented a novel model for sustainable HFSP and suggested an improved GA to solve this model. The applicability of these models and algorithms has been demonstrated with a case study. Cao et al. (2020b) aimed to optimize makespan and total energy cost in a multi-objective HFSP where the electricity prices are depended on TOU electricity tariffs, self-generation cost, and ongrid electrovalence. SPEA-II algorithm has been proposed to solve this problem and effectiveness of this algorithm has been 
indicated with a RCA of a Chinese company. Li et al. (2020b) presented a new version of MOEA/D and applied this method for hybrid flowshop lot streaming problem which is a scheduling problem that variable sub-lots constraint is considered.

Hybrid metaheuristics integrating more than one metaheuristic are also utilized to obtain solutions for HFSPs as seen in the following articles. Behnamian et al. (2010) studied on a HFSP with SDSTs and proposed a hybrid metaheuristic integrating ACO, SA, and VNS algorithms to solve this problem. They revealed that this algorithm outperforms RKGA and IA using test instances. Behnamian and Fatemi Ghomi (2011) presented a hybrid metaheuristic combining GA and VNS for multi-objective HFSP and they demonstrated that the proposed metaheuristic is superior to the random initial population SA algorithm by means of RGTPs. Behnamian et al. (2012) presented a hybrid metaheuristic combining ACO, VNS, and SA to solve HFSP with SDSTs and they demonstrated that the proposed metaheuristic outperforms some recent algorithms existing in the literature. Dai et al. (2013a) formulated a mathematical model for an energy-efficient HFSP and proposed an algorithm combining GA and SA algorithms to efficiently solve this problem for real size instances. Dai et al. (2013b) implemented a novel hybrid algorithm called IGAA integrating GA and SA algorithms to solve HFSP and demonstrated superiority of this algorithm according to existing two algorithms. Behnamian and Zandieh (2013) applied a hybrid metaheuristic integrating PSO, SA, and VNS algorithms to solve HFSP with SDSTs and learning effect and showed that this algorithm outperforms hybrid SA existing in the literature. Behnamian and Fatemi Ghomi (2014) presented a two-phase algorithm applying RKGA and PSO respectively for HFSP considering processing times, due dates and SDSTs as fuzzy parameters and indicated outperformance of the proposed algorithm. Behnamian et al. (2014) utilized a hybrid metaheuristic including PSO, VNS, and TS algorithms in order to obtain acceptable solutions for real size HFSPs providing tradeoff between exploration and exploitation and demonstrated better performance of this algorithm. Li et al. (2014b) proposed an algorithm combining PSO and ILS algorithms for HFSP considering PM operations. They evaluated applicability of this algorithm to real life industries by using well-known BPs and showed outperformance of it with respect to four existing algorithms. Zhou et al. (2018) after formulating a mathematical model for a reentrant HFSP, presented hybrid metaheuristic DE-eEDA integrating DE and EDA algorithms to solve this problem. They demonstrated effectiveness of the proposed metaheuristic via comparisons with different algorithms. Jubiz-Diaz et al. (2019) handled two multi-objective models integrating packaging size and HFS problems and aimed to minimize total cost and $\mathrm{kg}$ of $\mathrm{CO}_{2}$. They utilized HGA combining GRASP metaheuristic and GA to solve these models because of computational complexity. Lu et al. (2019) presented a novel MIP model for multi-objective HFSP with noise pollution criterion as different from existing literature and they proposed a new algorithm integrating GWO, cellular automata and VNS to balance exploration and exploitation. Besides, the effectiveness of this algorithm has been indicated compared with well-known algorithms. Ghaleb and Alharkan (2019) constructed a mathematical model for twostage nwt-HFSP to minimize total tardiness and suggested two PSO-based algorithms to cope with large instances. They showed superiority of the proposed algorithms with respect to benchmark algorithms from literature.

Similarly, hybrid metaheuristics integrating a metaheuristic and a heuristic (constructive, improvement or other) are effectively applied for HFSPs as seen in the following articles. Li et al. (2011) developed a MINLP model for HFSP and proposed a two-stage algorithm combining modified GA and local search to obtain near-optimal solutions for medium or large instances of this problem. They showed that the proposed algorithm is superior to some existing algorithms in terms of solution quality. Cho et al. (2011) handled multi-objective reentrant HFSP and applied pareto GA with different local search approaches to solve this problem. They compared the proposed algorithm with NSGA-II by using three performance measures and showed effectiveness of it. Xu et al. (2013) proposed an extended SFLA to minimize makespan in a HFSP and showed outperformance of this algorithm compared with different algorithms through BPs. Wang et al. (2013) applied an extended EDA in order to solve HFSP at real sizes and they indicated that this algorithm has better performance with respect to existing algorithms. Marichelvam et al. (2014a) utilized discrete FA to consider HFSP and determined that this algorithm is superior to GA, ACO, and SA metaheuristics in terms of solution quality. Pan and Dong (2014) applied a hybrid MBO algorithm to solve HFSP and demonstrated effectiveness of the proposed algorithm making comparisons with seven algorithms existing in the literature. Marichelvam et al. (2014b) considered HFSP with makespan minimization and proposed an algorithm integrating CS metaheuristic and NEH heuristic to solve this problem. They showed the effectiveness of the proposed algorithm making comparison with several other metaheuristics using real data from a furniture manufacturing company. Pan et al. (2014) performed an extended ABC algorithm utilizing 24 proposed heuristic algorithms to generate initial population and revealed outperformance of this algorithm with respect to different algorithms. Dasgupta and Das (2015) suggested a novel CS algorithm with the smallest position value rule for HFSP and demonstrated superiority of this algorithm with respect to different existing metaheuristics. Shen et al. (2016) applied an improved TLBO algorithm to solve reentrant HFSP with makespan and total tardiness objectives. The superiority of the proposed algorithm according to the IPG algorithm has been emphasized with numerical experiments realized with BPs. González-Neira et al. (2016) handled a HFSP consisting of ordinal and cardinal stages with SPTs. In the cardinal stage, they developed a MILP model to minimize TWT and solved this model by using a hybrid metaheuristic integrating GRASP algorithm and bottleneck heuristic. Pareto solutions of the cardinal stage have been qualified according to customer importance in the ordinal stage. Engin and Engin (2018) presented a memetic algorithm which integrates GA and local search so as to solve MTSP and indicated the effectiveness of it by comparing its performance with GA via BPs. Wu et al. (2018) applied a hybrid NSGA-II and variable local search algorithm to solve HFSP 
with variable processing times because of renewable energy and evaluated performance of this algorithm compared with NSGA-II. Peng et al. (2018a) examined a SCC problem considering machine breakdown and proposed an ABC algorithm extended with some modifications to effectively solve this problem. Qin et al. (2019) proposed a two-stage ACO algorithm to solve a printed circuit board scheduling problem with lot streaming and calendar constraint due to high complexity. Han et al. (2019a) presented an extended version of compact GA to contemplate HFSP with limited buffers and demonstrated efficiency of this algorithm with respect to existing algorithms. Li et al. (2019b) studied on HFSP with common due dates formulating a model and applied an extended GA (based on NSGA-II) algorithm so as to solve this problem. They showed that this algorithm is better than GA and PSO algorithms in terms of performance. Geng et al. (2019) focused on reentrant HFSP with on/off strategy and three objectives and proposed a MVO-based algorithm to effectively solve the considered problem in desired size. They compared it with other algorithms to evaluate its effectiveness. Hao et al. (2019) formulated distributed HFSP as a mathematical model and presented hybrid BSO to solve this problem. They evaluated performance of the proposed algorithm compared with some recent algorithms existing in the literature and showed effectiveness of it. Geng et al. (2020b) handled a multi-objective HFSP with dual resource constraints and presented a memetic algorithm to solve this problem. Performance of this algorithm has been compared with different multi-objective algorithms and it is stated that the proposed algorithm is efficient. Sun and Qi (2020) formulated a MIP model for HFSP to minimize makespan and delay cost and applied a hybrid metaheuristic integrating DE algorithm and local search to solve this problem. The robustness and outperformance of the proposed algorithm have been shown as a result of comparisons with four algorithms.

Besides, sometimes, algorithms combining more than one metaheuristics and heuristics are applied to handle HFSPs as seen in the following articles. Liao et al. (2012) addressed HFSP with makespan objective and proposed an extensive hybrid metaheuristic combining PSO, local search, bottleneck heuristic and SA to solve this problem. They assessed performance of the proposed algorithm compared with other algorithms through well-known benchmark problems (BPs). Peng et al. (2018b) applied an extended ABC algorithm to solve SCC problem which is a special case of HFS and demonstrated effectiveness of this algorithm with respect to several metaheuristics through comparisons. Zeng et al. (2018) focused on an environmentconscious HFSP which is formulated with a mathematical model and suggested a novel hybrid approach integrating NSGAII, TS and merging strategy to solve this problem. They showed the applicability of this algorithm for a real-life scheduling problem with comparisons. Zhou et al. (2019) implemented a novel algorithm integrating DE and PSO algorithms with Kalman filter and learning strategy in order to solve two-stage HFSP and demonstrated superiority of this algorithm as a result of comparisons with other algorithms. Xuan et al. (2019) presented a hybrid ABC-based metaheuristic so as to solve HFSP with the objective of TWCT for large instances. They verified the effectiveness of the proposed algorithm compared with single GA, GH and VNS algorithms. Marichelvam et al. (2020) examined a HFSP considering human factors and due to computational complexity they presented a hybrid algorithm integrating PSO algorithm with SPT rule, NEH heuristic and VNS algorithm to solve this problem. The superiority of the proposed algorithm was demonstrated with computational results.

Finally, the hybrid algorithms combining a metaheuristic and another method have been presented in the following articles. Lin et al. (2012) focused on dynamic reentrant HFSP and combined GA and AHP to solve this problem using real-life data of a repairing company. They showed that the obtained schedule with this algorithm is better than the manual schedule of the company. Wang et al. (2014b) proposed a two-phase simulation-based EDA in order to minimize makespan with SPTs under HFS environment and they showed that the proposed algorithm enables to obtain good results in terms of solution quality and computational time. Han et al. (2019b) applied a novel method integrating SA and Hopfield neural network algorithms to solve HFSP with a public buffer and indicated performance efficiency of this method compared with different algorithms. Zhang et al. (2019) formulated green HFSP with a multi-objective mathematical model which aims to minimize makespan and TEC and suggested an improved ABC algorithm to solve this problem. They compared the proposed algorithm with eight existing algorithms in the literature and evaluated the effectiveness of it. Chen et al. (2020) developed a MIP model for energyaware hybrid flowshop lot streaming problem and proposed an integrated algorithm including NSGA-II and MOEES algorithms to optimize makespan and energy consumption objectives simultaneously.

\subsubsection{Exact and Heuristic Algorithms}

In this sub-section, the articles applying an exact algorithm for small instances and a heuristic to solve large instances in a reasonable computational time have been briefly summarized as follows. Boudhar and Meziani (2010) aimed to handle HFSP with recirculation constraints and proposed three heuristic algorithms to solve this problem. They evaluated the effectiveness of these algorithms via RGTPs. Mao et al. (2014) focused on the SCC problem as a complex HFSP and developed a MIP model for this problem. They proposed a novel LR technique in order to solve the MIP model and demonstrated that the proposed method is more effective than classical LR approaches in terms of computational time and solution quality. Yu et al. (2017) focused on batching and scheduling problems in a two-stage hybrid flowshop and suggested a MILP model for this problem. Besides, they solved this model for small instances by CPLEX solver and presented three iterative algorithms as A1, $\mathrm{A} 2$, and $\mathrm{A} 3$ in order to obtain a good solution. It has been indicated that A3 is better than the other algorithms as a result of comparisons. Huang et al. (2017) developed a MILP model for two-stage reentrant HFSP with parallel batch-processing machines and it has been demonstrated by using CPLEX solver that the model is valid for small instances. They also proposed 
a $\mathrm{CH}$ called $\mathrm{H}$ and showed the effectiveness of this algorithm according to two improved heuristics with computational experiments. Asadi-Gangraj (2018) proposed a LR algorithm which includes two approaches as sub-problem simplification and dominance rules to solve sub-problems along with a MILP to consider HFSP and determined that the proposed approach is superior than MILP model in terms of computational time.

\subsubsection{Exact Algorithm and Metaheuristic}

In this sub-section, the articles using an exact algorithm to obtain optimal solution for HFSPs and a metaheuristic to cope with large instances have been briefly summarized as follows. The classification given in the sub-section 3.2.3 for metaheuristics has also been utilized in this sub-section.

Single metaheuristics are commonly utilized to make comparisons with exact algorithms as seen in the following articles. Javadian et al. (2012) developed a mathematical model enabled to solve small instances by LINGO software for HFSP and due to NP-hardness they proposed an immune algorithm-based metaheuristic for large-size problems. Besides, they demonstrated the superiority of the proposed algorithm in terms of computational time according to a mathematical model and a BP. Defersha and Chen (2012) suggested a mathematical model and GA for a HFSP with lot streaming and showed that the proposed algorithm enables to obtain better computational performance under a parallel computing environment. Shahvari et al. (2012) presented a MILP model for HFSP with group technology and solved this model by CPLEX solver for small instances. Besides, they proposed six TS-based metaheuristic algorithms in order to efficiently solve large instances and showed better performance of the best proposed algorithm in this study than an existing algorithm in the literature. Pargar and Zandieh (2012) proposed a mathematical model for HFSP including SDSTs and learning effect constraints and showed applicability of this model by solving via LINGO for six small problems. Because of NP-hard structure, they presented a metaheuristic named WFA for medium/large size problems and showed that this algorithm has better performance than RKGA. Costa et al. (2013) developed a MILP model for HFSP considering several constraints and solved it for small instances using CPLEX solver. Besides, they proposed a dual encoding-based metaheuristic to solve large-size problems and showed effectiveness of the proposed algorithm. Cheng et al. (2013) developed a MILP model for a HFSP with job splitting in the solar cell industry and proposed hybrid-coded GA to obtain near-optimal solutions. They showed that the proposed algorithm is better than the classical B\&B method and the heuristic approach applied by the company. Elmi and Topaloglu (2013) formulated a MILP model which enables optimally solving small instances for HFSP with some practical constraints and presented an improved SA algorithm for large problems due to computational complexity. They evaluated performance of this algorithm with numerical experiments. Huang and Yu (2013) focused on a two-stage HFSP motivated by the textile industry and obtained optimal solutions with LINGO software for small instances via mathematical models. Besides, they proposed an improved ACO algorithm to solve this problem for large instances effectively and determined that it provides better performance with respect to ACO and PSO algorithms. Yalaoui et al. (2013) analyzed a HFSP with specific constraints and constructed a MIP model to obtain exact solutions for this problem. Because of computational complexity, they presented GA and PSO algorithms with fuzzy extensions to solve real life problems and demonstrated effectiveness of fuzzy-based methods. Naderi and Yazdani (2014) presented a MILP model for lot streaming problem with SDSTs under HFS environment and obtained optimum solution up to 8 jobs in reasonable computational time through CPLEX solver. Besides, they proposed ICA for large instances and obtained better results compared to EDA, DEA and GH algorithms. Costa et al. (2014) considered HFSP with unrelated batch processors and some constraints developing a MILP model and solved this model via CPLEX solver for small instances. They also proposed a specific GA and revealed the effectiveness of this algorithm in terms of solution quality and computational time compared with some other metaheuristics for large instances. Nejati et al. (2014) proposed a MINLP model considering multi-job lot streaming problem and solved this model for small instances by means of LINGO software. Besides, they utilized GA and SA for large problems and obtained better solutions in terms of computational time with GA. Elmi and Topaloglu (2014) initially developed a MILP model with some practical constraints and then proposed a SA-based algorithm to cope with this HFSP in large instances. They determined that the proposed algorithm generates better solutions with respect to the MILP model in terms of computational time with a gap analysis. Huang et al. (2014) obtained optimal solutions of a two-stage reentrant HFSP for small instances with a mathematical model and also suggested an effective PSO algorithm which is superior to single PSO and ACO algorithms for this problem. Huang et al. (2015a) proposed an extended ACO algorithm with flexible update to solve two-stage nwt-HFSP and demonstrated that this algorithm is more effective than ACO algorithm for small problems, and is applicable for large size problems. Kheirandish et al. (2015) as well as developed a MILP model for two-stage assembly HFSP and solved it with LINGO software, they proposed discrete $\mathrm{ABC}$ algorithm and GA to solve this problem. It has been seen that the DABC algorithm has better performance in terms of CPU time. Huang et al. (2015b) after constructing an IP model for a two-stage HFSP, they proposed an enhanced PSO algorithm to solve this problem. Effectiveness and robustness of the proposed algorithm have been evaluated compared with IP model and classical PSO for small and large instances respectively. Defersha (2015) developed a mathematical model for HFS lot streaming problem and solved this problem for a small instance via CPLEX solver. Furthermore, the author proposed an SA algorithm with multiple search paths in order to obtain efficient solutions for this problem. Seidgar et al. (2016) developed a mathematical model that $\varepsilon$-constraint method is used to solve small instances 
optimally for two-stage assembly HFSP and conducted three multi-objective algorithms (NSGA-II, MOICA and NRGA) to cope with large instances. These algorithms have been evaluated through comparison metrics and the better performance of NRGA has been determined. Nejati et al. (2016) presented a MINLP model for a lot of streaming problems under a 2-stage assembly HFS environment and solved this model with LINGO. Besides, for large-size problems, they applied GA and SA and showed that SA generates better solutions in terms of computational time. Zabihzadeh and Rezaeian (2016) developed a MILP model for a HFSP including unrelated machines and several constraints and solved this model for small problems by using LINGO. They proposed two metaheuristic algorithms as ACO and GA for large instances and compared these algorithms to each other in terms of performance. Huang and Yu (2016) presented a mathematical model for two-stage HFSP considering maintenance deterioration constraint to provide cleaner production and solved this model for small problems. Afterwards, they proposed a cluster PSO algorithm to cope with large instances and showed robustness and effectiveness of this algorithm. Abdollahpour and Rezaian (2017) formulated nwt-HFSP with a mathematical model which is optimally solved by LINGO for small instances and also applied cloudy-based SA and AIS to handle large problems. The performance of these algorithms have been assessed and superiority of CSA algorithms has been shown to obtain better solutions. Tan et al. (2018) presented a MIP model solved by CPLEX for small instances to handle a two-stage HFSP and due to NP-hardness they also proposed an effective approach which utilizes VNS with a decomposition method to deal with this problem. Ying and Lin (2018) studied on a distributed HFSP with multiprocessor tasks and constructed a MILP model solved by GUROBI solver to express this problem. Besides, they proposed an effective and applicable self-tuning IG algorithm to solve this problem. Long et al. (2018) developed a mathematical model to examine a realistic SCC problem which is a well-known type of HFS and performed a statistically effective extended GA to solve this problem. Meng et al. (2019) examined an energy-oriented HFSP and formulated a MILP model to state this problem with on/off strategy and unrelated parallel machines. Afterwards, they presented a GA-based algorithm to solve this problem and suitability of this algorithm has been revealed compared with MILP model and three metaheuristics GA, SA, and MBO. Feng et al. (2019) developed a MILP model which enables the optimal solution for small problems to consider HF group scheduling problems with SDSTs and PM. GA has been presented to solve large instances and superiority of this algorithm has been demonstrated compared with CPLEX, PSO and CS by means of numerical experiments. Schulz et al. (2019) developed a MIP model for a multi-objective energy-aware HFSP and utilized epsilon method to determine optimal pareto front for small instances. Besides, they applied an ILS algorithm to identify pareto front for large instances and compared performance of this algorithm with NSGA-II. Guo et al. (2020) presented a MILP model to describe a combined batching and scheduling problem in the aluminum industry and obtained optimal solutions for small instances with CPLEX solver. They also proposed an improved version of the DE algorithm to solve industrial size problems and demonstrated effectiveness of this algorithm with comparisons.

The hybrid metaheuristics including more than one metaheuristic are applied with exact algorithms as seen in the following articles. Chen et al. (2013) formulated a HFSP with job splitting as a MILP model and proposed a hybrid metaheuristic integrating VNS and PSO algorithms for this problem. They demonstrated the effectiveness of the proposed metaheuristic according to B\&B method and the company's heuristic approach. Nikzad et al. (2015) developed a novel MILP model for a two-stage assembly HFSP and proposed a hybrid metaheuristic combining SA and ICA algorithms to solve this problem for real size instances. They compared this algorithm with LINGO and determined that it enables them to obtain good solutions in a reasonable time. Bozorgirad and Logendran (2016) presented a MILP model that is only solved by CPLEX solver for small instances to address a HFSP with some practical constraints and unrelated parallel machines. Besides, they proposed TS, SA and GA based metaheuristic algorithms to solve large problems and showed effectiveness of GA-based algorithms compared to local search-based TS and SA algorithms so as to obtain better solutions. Chamnanlor et al. (2017) presented a hybrid metaheuristic called as GACO which combines GA and ACO algorithm to solve reentrant HFSP with makespan minimization. They demonstrated outperformance of the proposed algorithm by means of test problems and a RCA of harddisk drive manufacturing. Bozorgnezhad et al. (2019) addressed a HFSP with worker assignment and developed a MILP model to evaluate this problem. While the model has been solved by CPLEX solver, in order to solve large instances they proposed a single PSO algorithm and a hybrid metaheuristic called as SPSO which integrates SA and PSO algorithms. It has been shown that SPSO outperforms single PSO as a result of this study. Garavito-Hernández et al. (2019) formulated a MILP model validated by using CPLEX solver for HFSP with setup constraints and presented an ICA-based metaheuristic which is relatively effective than MILP model for half of instances due to computational complexity. Raissi et al. (2019) presented a mathematical model for stochastic HFSP with PM and budget constraint as realistic characteristics. While the model has been optimally solved by CPLEX solver for small instances, they proposed two hybrid metaheuristics to obtain near-optimal solutions for large instances and evaluated performance of these algorithms in terms of objective value and computational time. Ding et al. (2020) proposed hybrid PSO algorithm for multi-objective HFSP with TOU electricity prices and variable speed levels and demonstrated superiority of this algorithm according to the MIP model and NSGA-II algorithm in terms of computational time and solution quality. Gong et al. (2020) studied on energy-oriented HFSP with worker flexibility and formulated a three-objective model to handle this problem. A hybrid evolutionary algorithm has been proposed to solve this problem in a reasonable time and it has been seen that the proposed algorithm is superior to CPLEX and two benchmark algorithms. 
The hybrid metaheuristics integrating a metaheuristic and a heuristic are compared with exact solutions in the following articles. Ziaeifar et al. (2012) presented a model integrating processor assignment and HFS problems and solved this model via LINGO for small instances. Besides, they proposed GA with an embedded heuristic to solve this problem in any size because of NP-hardness. Sheikh (2013) examined a HFSP with due window and time lag and presented a MILP model solved by LINGO 8 to state this problem. The author also proposed an extended GA and determined that this algorithm generates good solutions in terms of computational time and quality. Tadayon and Salmasi (2013) presented a mathematical model for HFSP with $r j$ and $M j$ constraints and solved optimally only a small part of test problems by CPLEX solver. They proposed four versions of the PSO algorithm for this problem owing to NP-hardness and showed effectiveness of PSO with local search approach. Keshavarz and Salmasi (2013) formulated a MILP model optimally solved by CPLEX for HFGSP and presented a memetic algorithm due to computational complexity. It has been demonstrated that the proposed algorithm is more effective than an existing algorithm TS and has suitable gap values compared to exact solution and lower bound. Yu (2014) presented a metaheuristic called twin-PSO to minimize total weighted earliness, tardiness and machine idle time under HFS environment and revealed that the proposed algorithm is superior to both integer programming and single PSO algorithm. Naderi et al. (2014) evaluated four MILP models related to HFSP in terms of size and computational complexity. Afterwards, while they obtained optimal solutions by CPLEX solver for small problems, a PSO-based hybrid metaheuristic has been proposed to solve large-size problems. The outperformance of the proposed algorithm has been shown compared with existing PSO algorithms and ILS algorithms in the literature. Li et al. (2015) presented a heuristic-search GA for a HFSP including both single and batch processing machines to minimize makespan and TWT objectives respectively. It has been seen that the proposed algorithm has computational efficiency compared to classical GA, and better solution quality with respect to CPLEX for large instances. Soltani and Karimi (2015) formulated a MILP for HFSP with realistic Mj and block constraints and due to NP-hard structure of this problem they applied SA and GA algorithms which utilize a heuristic to obtain an initial solution to solve it. The outperformance of SA algorithm in terms of solution quality and CPU time has been indicated. Zhang and Chen (2018) suggested an extended DE algorithm for large size problems, along with a MILP which enables to solve small instances optimally by CPLEX solver for reentrant HFSP with $M j$ constraint. They revealed that the proposed algorithm generates near-optimal solutions with a reasonable gap for small problems and is superior to two other metaheuristics. Azami et al. (2018) presented a MILP model which is suitable to solve small and medium size problems for a two-stage real-life composite manufacturing problem. They also proposed an effective GA-based algorithm which utilizes a heuristic for generating initial population to solve large instances because of computational complexity. Zohali et al. (2019a) studied economic lot sizing and scheduling problems and developed a MINLP model improving an existing model in the literature. Afterwards, they utilized a linearization technique and transformed this model to a MILP model. Although optimal solutions were obtained both of MINLP and MILP models through BARON and CPLEX solvers for small and medium instances, they proposed an algorithm integrating ILS and approximate function to solve large-size problems and showed effectiveness of it according to mathematical models and four existing metaheuristics in terms of computational performance. Zohali et al. (2019b) proposed two MILP models for lot scheduling problems with limited buffers and obtained optimal solutions for small and medium scale instances. They also proposed a metaheuristic algorithm named FFO for large scale instances and showed effectiveness of the proposed algorithm according to five existing algorithms. Chung and Chen (2019) evaluated a two-stage assembly HFSP including job splitting and due window constraints and developed a MILP model for this problem. They solved the model by using CPLEX solver for small instances and proposed Complete Immunoglobulin-based Artificial Immune System algorithm for large-size problems. Besides, they utilized five existing metaheuristics for comparison and showed effectiveness of the proposed algorithm. Li et al. (2020a) presented a MILP model to describe a HFSP with SDSTs and solved this model for small instances by CPLEX solver. They also proposed a hybrid ABC-based algorithm to cope with large instances and showed superiority of this algorithm with computational experiments and statistical analyses. Baxendale et al. (2020) formulated a variant of HFSP with a MIP model which provides optimal solution for some of the problem sizes and proposed the combined versions of SA and TS algorithms with a $\mathrm{CH}$ to solve large instances. They indicated that SA is superior to TS in terms of obtaining better results. Lei et al. (2020) considered HFSP with dynamic transport waiting times and constructed a model solved by GUROBI solver for this problem. A memetic algorithm has been presented which enables it to handle production and transportation concurrently and it has been demonstrated that the proposed algorithm is effective and suitable for industrial areas as a result of computational experiments. Costa et al. (2020) concerned with HFSP considering workforce constraints and presented a MILP model to obtain optimal solutions for small instances. A hybrid algorithm combining backtracking search algorithm and TS has been suggested to solve large instances and effectiveness of this algorithm has been evaluated through some non-parametric tests. Hasani et al. (2020) developed a MILP model for biobjective HFSP and utilized augmented $\varepsilon$-constraint method in order to evaluate performance of this model by using small instances. Besides, the NSGA-II algorithm has been suggested to obtain optimal or near optimal solutions due to NP-hardness. They also revealed the superiority of this algorithm according to the MIP model and SPEA-II with comparison metrics. The hybrid metaheuristics classified as other have been utilized with exact algorithms in the following articles. Tang and Song (2010) developed an IP model to evaluate a real life nwt-HFSP and proposed an algorithm integrating discrete PSO and nowait algorithm in stages for this problem. They showed the effectiveness of this algorithm by comparing the results with CPLEX solver and three heuristic algorithms. Golneshini and Fazlollahtabar (2019) formulated a MILP model for HFSP 
considering processing times and due dates as fuzzy parameters and solved this model with GAMS for small instances. Besides, hybrid versions of GA and PSO algorithms have been proposed for large problems and they evaluated performance of these proposed algorithms. Rooeinfar et al. (2019) presented a mathematical model for HFSP with SPTs and solved this model for small problems with CPLEX solver. They applied new hybrid methods combining computer simulation models and three well-known heuristics GA, SA, and PSO to solve this problem and they determined that the hybrid method is more effective than a single version of metaheuristics in terms of accuracy and speed. Li et al. (2020c) developed a machine position-based model for distributed HFSP to minimize makespan and determined lower bound for 19 small instances solving with CPLEX solver. Since this model is in NP-hard class, a hybrid metaheuristic called IABC has been applied to deal with large instances and outperformance of this algorithm has been revealed with comparisons.

\subsubsection{Heuristic and Metaheuristic}

In this sub-section, the articles applying heuristic and metaheuristic algorithms separately and comparing performance of them have been briefly summarized in the following. Ramezanian et al. (2017) proposed a MIP model for an integrated lot sizing and scheduling problem under HFS environment and aimed to minimize total cost consisting of production, inventory and external supply costs. Because of computational complexity, they applied rolling horizon heuristic and PSO algorithm to solve this problem. Shao et al. (2020) focused on distributed HFSP with makespan criterion and proposed NEH-based and IG-based algorithms to cope with this problem and it has been determined that these algorithms are effective to solve the problem.

\subsubsection{Exact Algorithm, Heuristic, and Metaheuristic}

In this sub-section, the articles using exact, heuristic, and metaheuristic algorithms separately in order to solve HFSPs and comparing them to each other have been briefly summarized in the following. Hekmatfar et al. (2011) developed a MIP model for two-stage reentrant HFSP and solved this model for eighteen small problems to evaluate the feasibility of it. Afterwards, they proposed four heuristic algorithms and a hybrid metaheuristic based on RKGA for large-sized problems and compared the performance of these algorithms to each other. Farahmand-Mehr et al. (2014) presented a MILP model to minimize makespan in HFSP and obtained an optimal solution by LINGO software. They also proposed three heuristic algorithms (Johnson, SPTCH, Palmer) and GA owing to the NP-hard feature of this problem and determined superiority of GA as a result of comparisons. Xiong et al. (2015) formulated a MIP model which is effective for small problems for a novel HFSP including assembly and differentiation operations and also presented two CHs (SPT, NEH) and three hybrid metaheuristics (GA-VNS, DE-VNS, EDA-VNS) to cope with medium and large instances. They realized computational experiments to assess performance of these algorithms by means of a lower bound. Komaki et al. (2016) studied a two-stage assembly HFSP with makespan objective and they proposed lower bounds, heuristics and two metaheuristic algorithms based on AIS so as to solve this problem. Feng et al. (2016) proposed exact, heuristic, and metaheuristic algorithms to solve two-stage HFSP with makespan minimization, and they evaluated performance of the proposed algorithms by means of test problems. Liu et al. (2017) presented a IP model to consider three-stage HFSP inspired from tempered glass manufacturing process and solved this model by CPLEX solver for small instances to minimize makespan. A CH and two metaheuristic algorithms GA and SA have been proposed to deal with large instances and they compared performance of these algorithms to each other to determine superior one. Oztop et al. (2019) obtained optimal solutions for small problems of HFSP with CPLEX by using a mathematical model. As well as a novel $\mathrm{CH}$, they applied four IG-based metaheuristic and variable block insertion heuristic to solve large instances. It has been shown that the proposed algorithms are effective in terms of solution quality and computational time. Oztop et al. (2020) developed a MILP and a CP model for energy-aware HFSP and augmented $\varepsilon$-constraint method has been applied to obtain optimal solutions for small problems. As well as a $\mathrm{CH}$, they presented seven single and hybrid metaheuristics to cope with large problems and evaluated efficiency of these algorithms through performance measures. Wang et al. (2020) addressed energy-conscious HFSP with a MIP model and applied an augmented $\varepsilon$-constraint method to obtain an exact solution for small instances. As well as problem-specific heuristic, they proposed two hybrid metaheuristics which combine both TS and ACO algorithms with CH for medium and large instances. Liu et al. (2020c) addressed HFSP with some practical constraints and constructed an IP model to minimize makespan and TECC simultaneously. While they obtained an exact solution with the $\varepsilon$-constraint method for small instances, a model based heuristic has been proposed and it has been shown that it outperforms $\varepsilon$-constraint method in terms of computational time. Finally, bi-objective DE algorithm has been applied to solve large problems and computational experiments demonstrate the superiority of this algorithm according to the $\varepsilon$ constraint method, the proposed heuristic, NSGA-II and the rule applied in manufacturing systems.

\subsubsection{Other Methods}

In this sub-section, the articles that the solution method couldn't be classified as exact, heuristic or metaheuristic has been briefly summarized as follows. Rahmani et al. (2013) considered new job arrivals as stochastic disruption in the HFS environment and proposed a new reactive approach to cope with this situation. They demonstrated that the proposed approach is superior to FIFO and penalty cost heuristics. Meghdari et al. (2015) presented a MIP model for HFSP with learning and forgetting effects and obtained a solution for an illustrative scheduling problem. Mollaei et al. (2019) formulated a bi-objective MILP model for HFSP considering processing times, setup costs and machine costs as uncertain parameters and utilized robust possibilistic programming approach to solve this problem. 
Table 3

A summary classification of included HFS articles

\begin{tabular}{|c|c|c|c|c|c|c|c|c|c|c|c|c|c|c|c|c|c|c|c|c|c|c|c|c|c|c|}
\hline \multirow{2}{*}{ Paper ID } & \multirow{2}{*}{ Year } & \multirow{2}{*}{$\begin{array}{l}\text { Stage } \\
\text { Type }\end{array}$} & \multicolumn{4}{|c|}{$\begin{array}{c}\text { Parallel } \\
\text { Machines }\end{array}$} & \multirow{2}{*}{$\begin{array}{c}\text { Contraint \& } \\
\text { Characteristics }\end{array}$} & \multicolumn{2}{|c|}{$\begin{array}{c}\text { Type of } \\
\text { Objective } \\
\end{array}$} & \multirow{2}{*}{$\begin{array}{l}\text { Objective } \\
\text { Function(s) }\end{array}$} & \multicolumn{10}{|c|}{ Solution Algorithm (s) } & \multicolumn{3}{|c|}{$\begin{array}{c}\text { Test } \\
\text { Instances }\end{array}$} & \multicolumn{3}{|c|}{$\begin{array}{c}\text { Type of } \\
\text { Parameters }\end{array}$} \\
\hline & & & 1 & Pm & $\mathbf{Q m}$ & $\mathbf{R m}$ & & $\mathbf{s}$ & M & & MIP & Eps & Othr & $\mathrm{CH}$ & IH & RT & DBA & Othr & Heuristic & Other & $\overline{R G T P}$ & BP & RCA & $\mathbf{D}$ & $\mathrm{s}$ & $\mathbf{F}$ \\
\hline Karimi et al. (2010) & 2010 & $\mathrm{~m}$ & & $\checkmark$ & & & SDST; fmls; skip & & $\checkmark$ & Cmax; TWT & & & & & & & & & $\checkmark$ & & $\checkmark$ & & & $\checkmark$ & & \\
\hline $\begin{array}{l}\text { Behnamian } \\
\text { et al. (2010) }\end{array}$ & 2010 & $\mathrm{~m}$ & & $\checkmark$ & & & SDST; skip & $\checkmark$ & & (TE+TT) & & & & & & & & & $\checkmark$ & & $\checkmark$ & & & $\checkmark$ & & \\
\hline Nishi et al. (2010) & 2010 & $\mathrm{~m}$ & & $\checkmark$ & & & & $\checkmark$ & & TWT & & & & & & $\checkmark$ & & & & & $\checkmark$ & & & $\checkmark$ & & \\
\hline $\begin{array}{l}\text { Boudhar and } \\
\text { Meziani (2010) }\end{array}$ & 2010 & 2 & $\checkmark$ & $\checkmark$ & & & rerc & $\checkmark$ & & Cmax & $\checkmark$ & & & & & & & $\checkmark$ & & & $\checkmark$ & & & $\checkmark$ & & \\
\hline $\begin{array}{l}\text { Tang and } \\
\text { Song (2010) }\end{array}$ & 2010 & $\mathrm{~m}$ & & $\checkmark$ & & & Batch; nwt & $\checkmark$ & & $\mathrm{Cmax}$ & $\checkmark$ & & & & & & & & $\checkmark$ & & $\checkmark$ & & $\checkmark$ & $\checkmark$ & & \\
\hline $\begin{array}{l}\text { Behnamian and } \\
\text { Zandieh (2011) }\end{array}$ & 2011 & $\mathrm{~m}$ & & $\checkmark$ & & & SDST; limwait; skip & $\checkmark$ & & $\left(\mathrm{TE}+\mathrm{TT}^{2}\right)$ & & & & & & & & & $\checkmark$ & & $\checkmark$ & & & $\checkmark$ & & \\
\hline Defersha (2011) & 2011 & 3 & & & & $\checkmark$ & $\begin{array}{l}\text { rm; batch; SDST; } \\
\text { Mj; lotstream; skip }\end{array}$ & $\checkmark$ & & Cmax & $\checkmark$ & & & & & & & & & & $\checkmark$ & & & $\checkmark$ & & \\
\hline $\begin{array}{l}\text { Solano-Charris } \\
\text { et al. (2011) }\end{array}$ & 2011 & 2 & & $\checkmark$ & & & SIST & $\checkmark$ & & $\alpha(\operatorname{Cmax})+(1-\alpha)(\mathrm{TCT})$ & & & & & & & & & $\checkmark$ & & $\checkmark$ & & & $\checkmark$ & & \\
\hline Luo et al. (2011) & 2011 & 2 & $\checkmark$ & $\checkmark$ & & & $\begin{array}{l}\text { SDST; batch; PM; } \\
\text { block (zero buffer) }\end{array}$ & $\checkmark$ & & $\mathrm{Cmax}$ & & & & & & & & & $\checkmark$ & & & & $\checkmark$ & $\checkmark$ & & \\
\hline $\begin{array}{l}\text { Behnamian and } \\
\text { Fatemi Ghomi (2011) }\end{array}$ & 2011 & $\mathrm{~m}$ & & & & $\checkmark$ & SDST; skip & & $\checkmark$ & $\begin{array}{l}\text { Cmax; Total resource allocation } \\
\text { cost }\end{array}$ & & & & & & & & & $\checkmark$ & & $\checkmark$ & & & $\checkmark$ & & \\
\hline Li et al. (2011) & 2011 & $\mathrm{~m}$ & & 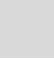 & $\checkmark$ & & $\begin{array}{l}\text { SDST; Mj; PM; } \\
\text { limited buffer }\end{array}$ & $\checkmark$ & & $\begin{array}{c}\alpha(\text { TWCT })+\beta(\text { Workload } \\
\text { deviation })\end{array}$ & & & & & & & & & $\checkmark$ & & $\checkmark$ & & $\checkmark$ & $\checkmark$ & & \\
\hline Cho et al. (2011) & 2011 & $\mathrm{~m}$ & & $\checkmark$ & & & rcrc & & $\checkmark$ & Cmax; TT & & & & & & & & & $\checkmark$ & & $\checkmark$ & & & $\checkmark$ & & \\
\hline $\begin{array}{l}\text { Hekmetfar } \\
\text { et al. (2011) }\end{array}$ & 2011 & 2 & & $\checkmark$ & & & SDST; rcrc & $\checkmark$ & & Cmax & $\checkmark$ & & & $\checkmark$ & & & & $\checkmark$ & $\checkmark$ & & $\checkmark$ & & & $\checkmark$ & & \\
\hline Javadian et al. (2012) & 2012 & $\mathrm{~m}$ & & $\checkmark$ & & & SDST; skip; time lag & $\checkmark$ & & Cmax & $\checkmark$ & & & & & & & & $\checkmark$ & & $\checkmark$ & & & $\checkmark$ & & \\
\hline $\begin{array}{l}\text { Defersha and } \\
\text { Chen (2012) }\end{array}$ & 2012 & $\mathrm{~m}$ & & 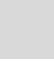 & & $\checkmark$ & $\begin{array}{l}\text { rm; batch; SDST; } \\
\text { Mj; lotstream; skip }\end{array}$ & $\checkmark$ & & Cmax & $\checkmark$ & & & & & & & & $\checkmark$ & & $\checkmark$ & & & $\checkmark$ & & \\
\hline Sawik (2012) & 2012 & $\mathrm{k}$ & $\checkmark$ & $\checkmark$ & & & $\begin{array}{l}\text { Batch; machine downtime; } \\
\text { cyclic; block (limited } \\
\text { buffer) }\end{array}$ & $\checkmark$ & & Cmax & $\checkmark$ & & & & & & & & & & $\checkmark$ & & & $\checkmark$ & & \\
\hline Gicquel et al. (2012) & 2012 & $\mathrm{k}$ & & $\checkmark$ & & & $\begin{array}{l}\text { SIST; batch; tj; sizeij } \\
\text { Block (zero buffer); nwt }\end{array}$ & $\checkmark$ & & TWT & $\checkmark$ & & & & & & & & & & $\checkmark$ & & & $\checkmark$ & & \\
\hline Cortes et al. (2012) & 2012 & $\mathrm{k}$ & $\checkmark$ & $\checkmark$ & & & Batch & 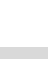 & $\checkmark$ & $\begin{array}{l}\text { Cmax; Resource usage; } \\
\text { Manufacturing lead time }\end{array}$ & & $\checkmark$ & & & & & & & & & & & $\checkmark$ & $\checkmark$ & & \\
\hline Wang and Choi (2012) & 2012 & $\mathrm{~m}$ & & $\checkmark$ & & & Brkdown & $\checkmark$ & & $\mathrm{Cmax}$ & & & & & & & $\checkmark$ & & & & $\checkmark$ & & & $\checkmark$ & & \\
\hline Choi and Wang (2012) & 2012 & $\mathrm{~m}$ & & $\checkmark$ & & & & $\checkmark$ & & Cmax & & & & & & & $\checkmark$ & & & & $\checkmark$ & & & & $\checkmark$ & \\
\hline Niu et al. (2012) & 2012 & $\mathrm{~m}$ & & $\checkmark$ & & & & $\checkmark$ & & MFT & & & & & & & & & $\checkmark$ & & $\checkmark$ & & & $\checkmark$ & & \\
\hline $\begin{array}{l}\text { Behnamian } \\
\text { et al. (2012) }\end{array}$ & 2012 & $\mathrm{~m}$ & & $\checkmark$ & & & SDST; skip & $\checkmark$ & & Cmax & & & & & & & & & $\checkmark$ & & $\checkmark$ & & & $\checkmark$ & & \\
\hline Liao et al. (2012) & 2012 & $\mathrm{~m}$ & & $\checkmark$ & & & SIST; tj & $\checkmark$ & & Cmax & & & & & & & & & $\checkmark$ & & & $\checkmark$ & & $\checkmark$ & & \\
\hline Lin et al. (2012) & 2012 & $\mathrm{k}$ & & & & & rerc & $\checkmark$ & & TWT & & & & & & & & & $\checkmark$ & & & & $\checkmark$ & $\checkmark$ & & \\
\hline Shahvari et al. (2012) & 2012 & $\mathrm{~m}$ & & $\checkmark$ & & & SDST; fmls & $\checkmark$ & & Cmax & $\checkmark$ & & & & & & & & $\checkmark$ & & $\checkmark$ & & & $\checkmark$ & & \\
\hline $\begin{array}{c}\text { Pargar and } \\
\text { Zandieh (2012) }\end{array}$ & 2012 & $\mathrm{~m}$ & & $\checkmark$ & & & SDST; LE & $\checkmark$ & & $\alpha(\mathrm{Cmax})+\beta(\mathrm{TT})$ & $\checkmark$ & & & & & & & & $\checkmark$ & & $\checkmark$ & & & $\checkmark$ & & \\
\hline Ziaeifar et al. (2012) & 2012 & $\mathrm{~m}$ & & $\checkmark$ & & & SIST & $\checkmark$ & & $\begin{array}{c}\alpha(\mathrm{Cmax})+ \\
(1-\alpha)(\text { Processor assignment } \\
\text { cost })\end{array}$ & $\checkmark$ & & & & & & & & $\checkmark$ & & $\checkmark$ & & & $\checkmark$ & & \\
\hline Li et al. (2013a) & 2013 & 2 & & $\checkmark$ & & $\checkmark$ & $\begin{array}{l}\text { rj; fmls (head group } \\
\text { constraint) }\end{array}$ & $\checkmark$ & & Cmax & & & & $\checkmark$ & & & & & & & $\checkmark$ & & & $\checkmark$ & & \\
\hline
\end{tabular}




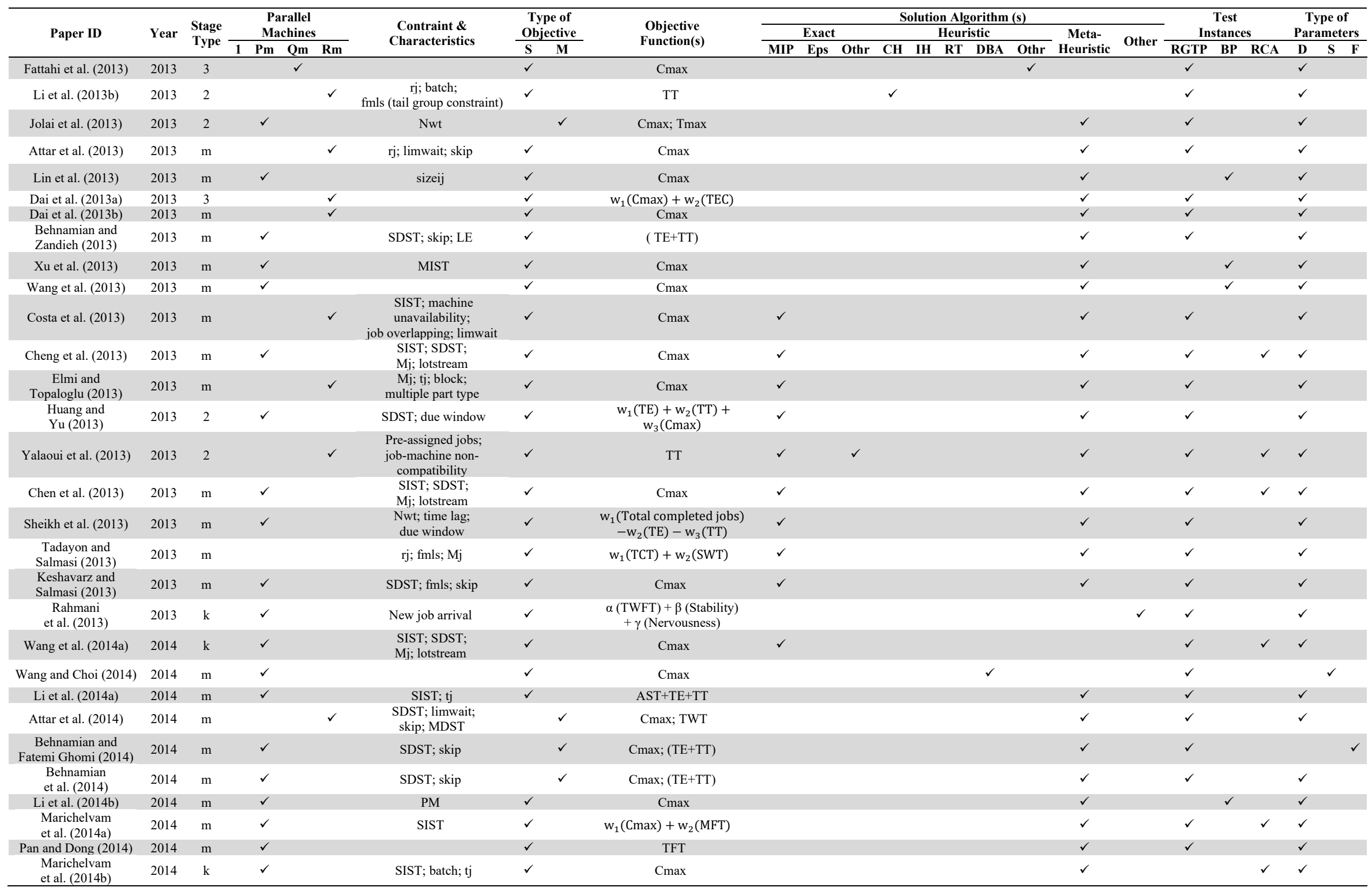


M. Çolak and G. A. Keskin / International Journal of Industrial Engineering Computations 13 (2022)

\begin{tabular}{|c|c|c|c|c|c|c|c|c|c|c|c|c|c|c|c|c|c|c|c|c|c|c|c|c|}
\hline \multirow{2}{*}{ Paper ID } & \multirow{2}{*}{ Year } & \multirow{2}{*}{$\begin{array}{l}\text { Stage } \\
\text { Type }\end{array}$} & \multicolumn{3}{|c|}{$\begin{array}{c}\text { Parallel } \\
\text { Machines } \\
\end{array}$} & \multirow{2}{*}{$\begin{array}{c}\text { Contraint \& } \\
\text { Characteristics }\end{array}$} & \multicolumn{2}{|c|}{$\begin{array}{c}\text { Type of } \\
\text { Objective }\end{array}$} & \multirow{2}{*}{$\begin{array}{c}\text { Objective } \\
\text { Function(s) }\end{array}$} & \multicolumn{10}{|c|}{ Solution Algorithm (s) } & \multicolumn{3}{|c|}{$\begin{array}{c}\text { Test } \\
\text { Instances }\end{array}$} & \multicolumn{2}{|c|}{$\begin{array}{c}\text { Type of } \\
\text { Parameters }\end{array}$} \\
\hline & & & 1 & Pm Qm & $\mathbf{R m}$ & & $\mathrm{s}$ & M & & MIP & Eps & Othr & $\mathrm{CH}$ & IH & RT & DBA & Othr & Heuristic & Other & $\begin{array}{l}\text { RGTP } \\
\end{array}$ & BP & RCA & & \\
\hline Pan et al. (2014) & 2014 & $\mathrm{~m}$ & & $\checkmark$ & & & $\checkmark$ & & Cmax & & & & & & & & & $\checkmark$ & & $\checkmark$ & $\checkmark$ & & $\checkmark$ & \\
\hline Wang et al. (2014b) & 2014 & $\mathrm{~m}$ & & $\checkmark$ & & & $\checkmark$ & & Cmax & & & & & & & & & $\checkmark$ & & $\checkmark$ & & & & $\checkmark$ \\
\hline Mao et al. (2014) & 2014 & $\mathrm{~m}$ & & $\checkmark$ & & $\begin{array}{l}\text { SIST; batch; } \\
\text { tj; fmls; prec }\end{array}$ & $\checkmark$ & & $\mathrm{TE}+\mathrm{TT}+\mathrm{SWT}$ & $\checkmark$ & & & & & $\checkmark$ & & & & & $\checkmark$ & & & $\checkmark$ & \\
\hline $\begin{array}{c}\text { Naderi and } \\
\text { Yazdani (2014) }\end{array}$ & 2014 & $\mathrm{~m}$ & & $\checkmark$ & & SDST; lotstream & $\checkmark$ & & TT & $\checkmark$ & & & & & & & & $\checkmark$ & & $\checkmark$ & & & $\checkmark$ & \\
\hline Costa et al. (2014) & 2014 & $\mathrm{~m}$ & & & $\checkmark$ & $\begin{array}{l}\text { SIST; batch; } \\
\text { fmls; } \mathrm{Mj}\end{array}$ & $\checkmark$ & & $\mathrm{Cmax}$ & $\checkmark$ & & & & & & & & $\checkmark$ & & $\checkmark$ & & & $\checkmark$ & \\
\hline Nejati et al. (2014) & 2014 & $\mathrm{~m}$ & & $\checkmark$ & & $\begin{array}{l}\text { SDST; batch; lotstream; } \\
\text { work shift constraint }\end{array}$ & $\checkmark$ & & TWCT & $\checkmark$ & & & & & & & & $\checkmark$ & & $\checkmark$ & & & $\checkmark$ & \\
\hline $\begin{array}{c}\text { Elmi and } \\
\text { Topaloglu (2014) }\end{array}$ & 2014 & $\mathrm{~m}$ & & & $\checkmark$ & $\begin{array}{c}\text { Mj; tj; block; } \\
\text { Multiple part type }\end{array}$ & $\checkmark$ & & Cmax & $\checkmark$ & & & & & & & & $\checkmark$ & & $\checkmark$ & & & $\checkmark$ & \\
\hline Huang et al. (2014) & 2014 & 2 & & & & rcrc; due window & $\checkmark$ & & $w_{1}(\mathrm{TE})+w_{2}(\mathrm{TT})$ & $\checkmark$ & & & & & & & & $\checkmark$ & & $\checkmark$ & & & $\checkmark$ & \\
\hline Yu (2014) & 2014 & 2 & & & & SDST & $\checkmark$ & & $\begin{array}{c}w_{1}(\mathrm{TE})+w_{2}(\mathrm{TT})+ \\
w_{3}(\mathrm{TMIT})\end{array}$ & $\checkmark$ & & & & & & & & $\checkmark$ & & $\checkmark$ & & & $\checkmark$ & \\
\hline Naderi et al. (2014) & 2014 & $\mathrm{~m}$ & & $\checkmark$ & & skip & $\checkmark$ & & Cmax & $\checkmark$ & & & & & & & & $\checkmark$ & & $\checkmark$ & & & $\checkmark$ & \\
\hline $\begin{array}{l}\text { Farahmand-Mehr } \\
\text { et al. (2014) }\end{array}$ & 2014 & $\mathrm{~m}$ & & $\checkmark$ & & SDST; skip; time lag & $\checkmark$ & & Cmax & $\checkmark$ & & & $\checkmark$ & & & & $\checkmark$ & $\checkmark$ & & $\checkmark$ & & & $\checkmark$ & \\
\hline Li et al. (2015) & 2015 & $\mathrm{~m}$ & & & $\checkmark$ & rj; SIST; batch & $\checkmark$ & & Cmax; TWT & $\checkmark$ & & & & & & & & $\checkmark$ & & $\checkmark$ & & & $\checkmark$ & \\
\hline Huang et al. (2015a) & 2015 & 2 & & & & SIST; nwt; time window & $\checkmark$ & & $w_{1}(\mathrm{TE})+w_{2}(\mathrm{TT})$ & $\checkmark$ & & & & & & & & $\checkmark$ & & $\checkmark$ & & & $\checkmark$ & \\
\hline Nikzad et al. (2015) & 2015 & 2 & $\checkmark$ & & $\checkmark$ & SDST; batch; $\mathrm{Mj}$; assembly & $\checkmark$ & & Cmax & $\checkmark$ & & & & & & & & $\checkmark$ & & $\checkmark$ & & & $\checkmark$ & \\
\hline Wang et al. (2015) & 2015 & 2 & $\checkmark$ & $\checkmark$ & & Nwt (zero buffer) & $\checkmark$ & & $\mathrm{Cmax}$ & & & $\checkmark$ & & & & & & & & $\checkmark$ & & & $\checkmark$ & \\
\hline Jiang et al. (2015) & 2015 & $\mathrm{~m}$ & & $\checkmark$ & & $\begin{array}{c}\text { SIST; batch; tj; skip; } \\
\text { controllable processing times }\end{array}$ & $\checkmark$ & & $\begin{array}{c}\text { SWT }+(\text { TE }+ \text { TT })+\text { Adjusting } \\
\text { cost }\end{array}$ & & & & & & & $\checkmark$ & & & & $\checkmark$ & & & $\checkmark$ & \\
\hline Lee et al. (2015) & 2015 & 2 & $\checkmark$ & $\checkmark$ & & SDST & $\checkmark$ & & Cmax & & & & $\checkmark$ & $\checkmark$ & & & & & & $\checkmark$ & & & $\checkmark$ & \\
\hline $\begin{array}{l}\text { Ghodratnama } \\
\text { et al. (2015) }\end{array}$ & 2015 & $\mathrm{k}$ & & $\checkmark$ & & & & $\checkmark$ & $\begin{array}{l}\text { TWT; Total incurred cost; } \\
\text { Total purchasing cost }\end{array}$ & & & & & & & & & $\checkmark$ & & $\checkmark$ & & & $\checkmark$ & \\
\hline $\begin{array}{l}\text { Dasgupta and } \\
\text { Das (2015) }\end{array}$ & 2015 & $\mathrm{k}$ & & $\checkmark$ & & SIST & $\checkmark$ & & $w_{1}(\mathrm{Cmax})+w_{2}(\mathrm{MFT})$ & & & & & & & & & $\checkmark$ & & & $\checkmark$ & $\checkmark$ & $\checkmark$ & \\
\hline $\begin{array}{l}\text { Kheirandish } \\
\text { et al. (2015) }\end{array}$ & 2015 & 2 & $\checkmark$ & & $\checkmark$ & SDST; batch; assembly & $\checkmark$ & & Cmax & $\checkmark$ & & & & & & & & $\checkmark$ & & $\checkmark$ & & & $\checkmark$ & \\
\hline Huang et al. (2015b) & 2015 & 2 & & $\checkmark$ & & & $\checkmark$ & & $w_{1}(\mathrm{TE})+w_{2}(\mathrm{TT})$ & $\checkmark$ & & & & & & & & $\checkmark$ & & $\checkmark$ & & & $\checkmark$ & \\
\hline Defersha (2015) & 2015 & $\mathrm{~m}$ & & & $\checkmark$ & $\begin{array}{l}\text { rm; SDST; batch; } \\
\text { Mj; lotstream; skip }\end{array}$ & $\checkmark$ & & Cmax & $\checkmark$ & & & & & & & & $\checkmark$ & & $\checkmark$ & & & $\checkmark$ & \\
\hline $\begin{array}{l}\text { Soltani and } \\
\text { Karimi (2015) }\end{array}$ & 2015 & 3 & & & $\checkmark$ & $\begin{array}{c}\text { Mj; cyclic; } \\
\text { block (limited buffer) }\end{array}$ & $\checkmark$ & & Cycle time & $\checkmark$ & & & & & & & & $\checkmark$ & & $\checkmark$ & & & $\checkmark$ & \\
\hline Xiong et al. (2015) & 2015 & 3 & $\checkmark$ & & $\checkmark$ & Mj; assembly & $\checkmark$ & & TFT & $\checkmark$ & & & $\checkmark$ & & & & & $\checkmark$ & & $\checkmark$ & & & $\checkmark$ & \\
\hline $\begin{array}{l}\text { Meghdari } \\
\text { et al. (2015) }\end{array}$ & 2015 & 2 & & $\checkmark$ & & SDST; LE/FE & $\checkmark$ & & $\alpha \operatorname{Cmax}+(1-\alpha) \operatorname{Tmax}$ & & & & & & & & & & $\checkmark$ & $\checkmark$ & & & $\checkmark$ & \\
\hline $\begin{array}{c}\text { Rahmani and } \\
\text { Ramezanian (2016) }\end{array}$ & 2016 & $\mathrm{k}$ & & $\checkmark$ & & SDST; new job arrival & $\checkmark$ & & TWT & & & & & & & & & $\checkmark$ & & & & $\checkmark$ & $\checkmark$ & \\
\hline Tang et al. (2016) & 2016 & $\mathrm{~m}$ & & & $\checkmark$ & Brkdown; new job arrival & & $\checkmark$ & Cmax; TEC & & & & & & & & & $\checkmark$ & & & $\checkmark$ & & $\checkmark$ & \\
\hline
\end{tabular}




\begin{tabular}{|c|c|c|c|c|c|c|c|c|c|c|c|c|c|c|c|c|c|c|c|c|c|c|c|c|c|}
\hline \multirow{3}{*}{ Paper ID } & \multirow{3}{*}{ Year } & \multirow{3}{*}{$\begin{array}{l}\text { Stage } \\
\text { Type }\end{array}$} & \multirow{2}{*}{\multicolumn{4}{|c|}{$\begin{array}{c}\text { Parallel } \\
\text { Machines }\end{array}$}} & \multirow{3}{*}{$\begin{array}{l}\text { Contraint \& } \\
\text { Characteristics }\end{array}$} & \multirow{2}{*}{\multicolumn{2}{|c|}{$\begin{array}{c}\text { Type of } \\
\text { Objective } \\
\end{array}$}} & \multirow{3}{*}{$\begin{array}{c}\text { Objective } \\
\text { Function(s) }\end{array}$} & \multicolumn{10}{|c|}{$\begin{array}{l}\text { Solution Algorithm (s) } \\
\end{array}$} & \multirow{2}{*}{\multicolumn{3}{|c|}{$\begin{array}{c}\text { Test } \\
\text { Instances }\end{array}$}} & \multirow{2}{*}{\multicolumn{2}{|c|}{$\begin{array}{c}\text { Type of } \\
\text { Parameters }\end{array}$}} \\
\hline & & & & & & & & & & & \multicolumn{3}{|c|}{ Exact } & \multicolumn{5}{|c|}{ Heuristic } & \multirow{2}{*}{$\begin{array}{c}\text { Meta- } \\
\text { Heuristic }\end{array}$} & \multirow{2}{*}{ Other } & & & & & \\
\hline & & & 1 & $\mathbf{P m}$ & Qm & $\mathbf{R m}$ & & $\mathbf{S}$ & $\mathbf{M}$ & & MIP & Eps & Othr & CH & IH & RT & DBA & Othr & & & RGTP & BP & RCA & D & $\mathbf{S} \quad \mathbf{F}$ \\
\hline Yan et al. (2016) & 2016 & $\mathrm{k}$ & & & & $\checkmark$ & $\mathrm{tj} ;$ MDST & $\checkmark$ & & $\alpha(\mathrm{Cmax})+(1-\alpha)(\mathrm{TEC})$ & & & & & & & & & $\checkmark$ & & $\checkmark$ & & & $\checkmark$ & \\
\hline Pan (2016) & 2016 & $\mathrm{~m}$ & & $\checkmark$ & & & SIST; $\mathrm{tj}$ & $\checkmark$ & & $w_{1}(\mathrm{Cmax})+w_{2}(\mathrm{SWT})$ & & & & & & & & & $\checkmark$ & & $\checkmark$ & & $\checkmark$ & $\checkmark$ & \\
\hline Shen et al. (2016) & 2016 & $\mathrm{~m}$ & & $\checkmark$ & & & rerc & & $\checkmark$ & Cmax; TT & & & & & & & & & $\checkmark$ & & & $\checkmark$ & & $\checkmark$ & \\
\hline $\begin{array}{l}\text { González-Neira } \\
\text { et al. (2016) }\end{array}$ & 2016 & $\mathrm{k}$ & & $\checkmark$ & & & & & $\checkmark$ & TWT; Customer importance & & & & & & & & & $\checkmark$ & & & $\checkmark$ & & & $\checkmark$ \\
\hline Seidgar et al. (2016) & 2016 & 2 & $\checkmark$ & & & $\checkmark$ & PM; assembly & & $\checkmark$ & Cmax; System unavailability & & $\checkmark$ & & & & & & & $\checkmark$ & & $\checkmark$ & & & $\checkmark$ & \\
\hline Nejati et al. (2016) & 2016 & 2 & & $\checkmark$ & & & $\begin{array}{c}\text { Batch; lotstream; } \\
\text { work shift constraint; } \\
\text { assembly }\end{array}$ & $\checkmark$ & & TWCT & $\checkmark$ & & & & & & & & $\checkmark$ & & $\checkmark$ & & & $\checkmark$ & \\
\hline $\begin{array}{l}\text { Zabihzadeh and } \\
\text { Rezaeian (2016) }\end{array}$ & 2016 & $\mathrm{~m}$ & & & & $\checkmark$ & $\begin{array}{c}\mathrm{rj} ; \mathrm{Mj} ; \mathrm{tj} \\
\text { block (zero buffer) }\end{array}$ & $\checkmark$ & & Cmax & $\checkmark$ & & & & & & & & $\checkmark$ & & $\checkmark$ & & & $\checkmark$ & \\
\hline $\begin{array}{c}\text { Huang and } \\
\text { Yu (2016) }\end{array}$ & 2016 & 2 & & $\checkmark$ & & & $\begin{array}{l}\text { Maintenance } \\
\text { deterioration }\end{array}$ & $\checkmark$ & & Cmax & $\checkmark$ & & & & & & & & $\checkmark$ & & $\checkmark$ & & & $\checkmark$ & \\
\hline $\begin{array}{l}\text { Bozorgirad and } \\
\text { Logendran (2016) }\end{array}$ & 2016 & $\mathrm{~m}$ & & & & $\checkmark$ & $\begin{array}{l}\text { rj; rm; SDST; fmls; } \\
\text { Mj; skip; LE; MDST }\end{array}$ & $\checkmark$ & & $\alpha($ TWCT $)+\beta($ TWT $)$ & $\checkmark$ & & & & & & & & $\checkmark$ & & $\checkmark$ & & & $\checkmark$ & \\
\hline Komaki et al. (2016) & 2016 & 3 & $\checkmark$ & $\checkmark$ & & & Assembly & $\checkmark$ & & Cmax & $\checkmark$ & & & & & & & $\checkmark$ & $\checkmark$ & & $\checkmark$ & & & $\checkmark$ & \\
\hline Feng et al. (2016) & 2016 & 2 & $\checkmark$ & $\checkmark$ & & & Robust scheduling & $\checkmark$ & & Cmax & & & $\checkmark$ & & $\checkmark$ & & & & $\checkmark$ & & $\checkmark$ & & & 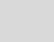 & $\checkmark$ \\
\hline $\begin{array}{l}\text { Ramezanian } \\
\text { et al. (2017) }\end{array}$ & 2017 & $\mathrm{~m}$ & & $\checkmark$ & & & $\begin{array}{l}\text { SDST; lot sizing; } \\
\text { machine capacity }\end{array}$ & $\checkmark$ & & PC + IC + External supply cost & & & & & & & & $\checkmark$ & $\checkmark$ & & $\checkmark$ & & $\checkmark$ & $\checkmark$ & \\
\hline Cui and Luo (2017) & 2017 & $\mathrm{~m}$ & & $\checkmark$ & & & SDST; batch; tj & $\checkmark$ & & $\mathrm{TE}+\mathrm{TT}+\mathrm{SWT}$ & & & & $\checkmark$ & & $\checkmark$ & & & & & $\checkmark$ & & & $\checkmark$ & \\
\hline Shim et al. (2017) & 2017 & $\mathrm{~m}$ & & $\checkmark$ & & & $\begin{array}{l}\text { SDST; lot sizing; } \\
\text { Bottleneck-focused } \\
\text { scheduling }\end{array}$ & $\checkmark$ & & TT & & & & $\checkmark$ & & & & & & & $\checkmark$ & & & $\checkmark$ & \\
\hline Zhang et al. (2017) & 2017 & $\mathrm{~m}$ & & $\checkmark$ & & & Lotstream & $\checkmark$ & & TFT & & & & & & & & & $\checkmark$ & & $\checkmark$ & & & $\checkmark$ & \\
\hline Yu et al. (2017) & 2017 & 2 & & $\checkmark$ & & & SIST; batch & $\checkmark$ & & TT & $\checkmark$ & & & & & & & $\checkmark$ & & & $\checkmark$ & & & $\checkmark$ & \\
\hline Huang et al. (2017) & 2017 & 2 & & $\checkmark$ & & & $\begin{array}{c}\text { rj; batch; } \\
\text { job weight; rcrc }\end{array}$ & $\checkmark$ & & Cmax & $\checkmark$ & & & $\checkmark$ & & & & & & & $\checkmark$ & & & $\checkmark$ & \\
\hline $\begin{array}{l}\text { Abdollahpour and } \\
\text { Rezaian (2017) }\end{array}$ & 2017 & $\mathrm{~m}$ & & $\checkmark$ & & & $\begin{array}{l}\text { rj; SDST; nwt; } \\
\text { machine capacity }\end{array}$ & $\checkmark$ & & $\begin{array}{c}\mathrm{w}_{1}(\mathrm{TE})+\mathrm{w}_{2}(\mathrm{TT})+ \\
\mathrm{w}_{3} \text { (Missed orders) }+ \\
\mathrm{w}_{4} \text { (Incomplete orders) }\end{array}$ & $\checkmark$ & & & & & & & & $\checkmark$ & & $\checkmark$ & & & $\checkmark$ & \\
\hline $\begin{array}{l}\text { Chamnanlor } \\
\text { et al. (2017) }\end{array}$ & 2017 & $\mathrm{~m}$ & & & $\checkmark$ & & $\begin{array}{l}\text { SIST; fmls; Mj; } \\
\text { rcrc; time window }\end{array}$ & $\checkmark$ & & Cmax & $\checkmark$ & & & & & & & & $\checkmark$ & & $\checkmark$ & & $\checkmark$ & $\checkmark$ & \\
\hline Liu et al. (2017) & 2017 & 3 & $\checkmark$ & & $\checkmark$ & & $\begin{array}{l}\text { Batch; } \mathrm{Mj} ; \\
\text { limited buffer }\end{array}$ & $\checkmark$ & & Cmax & $\checkmark$ & & & $\checkmark$ & & & & & $\checkmark$ & & $\checkmark$ & & & $\checkmark$ & \\
\hline $\begin{array}{l}\text { Engin and } \\
\text { Engin (2018) }\end{array}$ & 2018 & $\mathrm{~m}$ & & $\checkmark$ & & & $\begin{array}{l}\text { sizeij; common time } \\
\text { window }\end{array}$ & $\checkmark$ & & $w_{1}(\mathrm{TE})+w_{2}(\mathrm{TT})$ & & & & & & & & & $\checkmark$ & & & $\checkmark$ & & $\checkmark$ & \\
\hline Wang et al. (2018) & 2018 & 2 & $\checkmark$ & $\checkmark$ & & & $\begin{array}{l}\text { SDST; nwt; simultaneous } \\
\text { processing machines }\end{array}$ & $\checkmark$ & & Total processing time & & & & $\checkmark$ & & & & & & & $\checkmark$ & & & $\checkmark$ & \\
\hline Zhou et al. (2018) & 2018 & $\mathrm{~m}$ & & $\checkmark$ & & & rerc & $\checkmark$ & & TWCT & & & & & & & & & $\checkmark$ & & $\checkmark$ & & & $\checkmark$ & \\
\hline Wu et al. (2018) & 2018 & $\mathrm{~m}$ & & & & $\checkmark$ & Variable processing times & & $\checkmark$ & Cmax; Total carbon footprint & & & & & & & & & $\checkmark$ & & & & $\checkmark$ & $\checkmark$ & \\
\hline Peng et al. (2018a) & 2018 & 3 & & $\checkmark$ & & & $\begin{array}{l}\text { SIST; brkdown; } \mathrm{tj} ; \\
\text { controllable processing } \\
\text { times }\end{array}$ & $\checkmark$ & & $\begin{array}{c}\mathrm{w}_{1}(\mathrm{AST})+\mathrm{w}_{2}(\mathrm{TE})+ \\
\mathrm{w}_{3}(\mathrm{TT})++\mathrm{w}_{4}(\text { Cast break) }+ \\
\mathrm{w}_{5} \text { (Starting time difference) }+ \\
\mathrm{w}_{6} \text { (The number of operations) }\end{array}$ & & & & & & & & & $\checkmark$ & & $\checkmark$ & & $\checkmark$ & $\checkmark$ & \\
\hline Peng et al. (2018b) & 2018 & 3 & & $\checkmark$ & & & SIST; tj & $\checkmark$ & & $w_{1}(\mathrm{AST})+w_{2}(\mathrm{TE})+w_{3}(\mathrm{TT})$ & & & & & & & & & $\checkmark$ & & $\checkmark$ & & & $\checkmark$ & \\
\hline Zeng et al. (2018) & 2018 & 2 & & & & $\checkmark$ & SIST; batch & & $\checkmark$ & Cmax; TEC; Material wastage & & & & & & & & & $\checkmark$ & & $\checkmark$ & & & $\checkmark$ & \\
\hline Asadi-Gangraj (2018) & 2018 & $\mathrm{~m}$ & & & & $\checkmark$ & SIST & $\checkmark$ & & Cmax & $\checkmark$ & & & & & $\checkmark$ & & & & & $\checkmark$ & & & $\checkmark$ & \\
\hline
\end{tabular}




\begin{tabular}{|c|c|c|c|c|c|c|c|c|c|c|c|c|c|c|c|c|c|c|c|c|c|c|c|c|c|}
\hline \multirow{2}{*}{ Paper ID } & \multirow{2}{*}{ Year } & \multirow{2}{*}{$\begin{array}{l}\text { Stage } \\
\text { Type }\end{array}$} & \multicolumn{4}{|c|}{$\begin{array}{c}\text { Parallel } \\
\text { Machines }\end{array}$} & \multirow{2}{*}{$\begin{array}{c}\text { Contraint \& } \\
\text { Characteristics }\end{array}$} & \multicolumn{2}{|c|}{$\begin{array}{c}\text { Type of } \\
\text { Objective }\end{array}$} & \multirow{2}{*}{$\begin{array}{c}\text { Objective } \\
\text { Function(s) }\end{array}$} & \multicolumn{9}{|c|}{$\begin{array}{l}\text { Solution Algorithm (s) } \\
\text { Heuristic }\end{array}$} & \multicolumn{3}{|c|}{$\begin{array}{c}\text { Test } \\
\text { Instances } \\
\end{array}$} & \multicolumn{3}{|c|}{$\begin{array}{c}\text { Type of } \\
\text { Parameters }\end{array}$} \\
\hline & & & 1 & $\mathbf{P m}$ & Qm & $\mathbf{R m}$ & & $\mathbf{s}$ & M & & MIP & Eps & Othr & $\mathrm{CH}$ & IH RT & DBA & Othr & Heuristic & Other & RGTP & BP & RCA & D & $\mathbf{s}$ & $\mathbf{F}$ \\
\hline Tan et al. (2018) & 2018 & 2 & & $\checkmark$ & & & $\begin{array}{l}\text { rj; batch; } \\
\text { fmls (incompatible job families) }\end{array}$ & $\checkmark$ & & TWT & $\checkmark$ & & & & & & & $\checkmark$ & & $\checkmark$ & & & $\checkmark$ & & \\
\hline Ying and Lin (2018) & 2018 & $\mathrm{~m}$ & & $\checkmark$ & & & sizeij; distributed & $\checkmark$ & & Cmax & $\checkmark$ & & & & & & & $\checkmark$ & & $\checkmark$ & & & $\checkmark$ & & \\
\hline Long et al. (2018) & 2018 & $\mathrm{~m}$ & & $\checkmark$ & & & $\begin{array}{c}\text { SIST; tj; skip; } \\
\text { adjustable processing times }\end{array}$ & $\checkmark$ & & $\begin{array}{c}\mathrm{w}_{1}(\mathrm{Cmax})+\mathrm{w}_{2}(\mathrm{SWT})+ \\
\mathrm{w}_{3}(\text { Total processing time deviation })\end{array}$ & $\checkmark$ & & & & & & & $\checkmark$ & & $\checkmark$ & & & $\checkmark$ & & \\
\hline $\begin{array}{l}\text { Zhang and } \\
\text { Chen (2018) }\end{array}$ & 2018 & $\mathrm{~m}$ & & $\checkmark$ & & & $\mathrm{Mj} ;$ rcrc & $\checkmark$ & & TT & $\checkmark$ & & & & & & & $\checkmark$ & & $\checkmark$ & & & $\checkmark$ & & \\
\hline Azami et al. (2018) & 2018 & 2 & & $\checkmark$ & $\checkmark$ & $\checkmark$ & $\begin{array}{l}\text { Batch; limwait } \\
\text { block (limited buffer) }\end{array}$ & $\checkmark$ & & $\begin{array}{l}\text { The number of jobs that can be } \\
\text { assigned to curing process } 1\end{array}$ & $\checkmark$ & & & & & & & $\checkmark$ & & & & $\checkmark$ & $\checkmark$ & & \\
\hline Kurdi (2019) & 2019 & $\mathrm{~m}$ & & $\checkmark$ & & & sizeij & $\checkmark$ & & $\mathrm{Cmax}$ & & & & & & & & $\checkmark$ & & & $\checkmark$ & & $\checkmark$ & & \\
\hline Meng et al. (2019) & 2019 & $\mathrm{~m}$ & & & & $\checkmark$ & On/off & $\checkmark$ & & TEC & $\checkmark$ & & & & & & & $\checkmark$ & & $\checkmark$ & & & $\checkmark$ & & \\
\hline Feng et al. (2019) & 2019 & $\mathrm{~m}$ & & $\checkmark$ & & & $\begin{array}{c}\text { SDST; fimls; PM; } \\
\text { skip; multiple setups }\end{array}$ & $\checkmark$ & & Cmax & $\checkmark$ & & & & & & & $\checkmark$ & & $\checkmark$ & & & $\checkmark$ & & \\
\hline Luo et al. (2019) & 2019 & $\mathrm{~m}$ & & & & $\checkmark$ & rj; new job arrival & $\checkmark$ & & $\mathrm{w}(\mathrm{TT})+\mathrm{Cmax}$ & & & & & & & & $\checkmark$ & & $\checkmark$ & & & $\checkmark$ & & \\
\hline $\begin{array}{l}\text { Jubiz-Diaz } \\
\text { et al. (2019) }\end{array}$ & 2019 & $\mathrm{k}$ & & $\checkmark$ & & & SDST; lot sizing & & $\checkmark$ & Total cost; $\mathrm{Kg}_{\text {of }} \mathrm{CO}_{2}$ & & & & & & & & $\checkmark$ & & & & $\checkmark$ & $\checkmark$ & & \\
\hline Lu et al. (2019) & 2019 & $\mathrm{~m}$ & & & $\checkmark$ & & & & $\checkmark$ & Cmax; TEC; Noise pollution & & & & & & & & $\checkmark$ & & $\checkmark$ & $\checkmark$ & & $\checkmark$ & & \\
\hline $\begin{array}{l}\text { Ghaleb and } \\
\text { Alharkan (2019) }\end{array}$ & 2019 & 2 & & $\checkmark$ & & & SIST; nwt; & $\checkmark$ & & TT & & & & & & & & $\checkmark$ & & $\checkmark$ & & & $\checkmark$ & & \\
\hline Qin et al. (2019) & 2019 & $\mathrm{k}$ & & & & $\checkmark$ & $\begin{array}{l}\text { SDST; batch; skip; } \\
\text { lotstream; } \\
\text { calendar constraint }\end{array}$ & $\checkmark$ & & Cmax & & & & & & & & $\checkmark$ & & & & $\checkmark$ & $\checkmark$ & & \\
\hline Han et al. (2019a) & 2019 & $\mathrm{~m}$ & & $\checkmark$ & & & SIST; block (limited buffer) & $\checkmark$ & & $\begin{array}{l}\text { Cmax; TMIT; SWT; Total device } \\
\text { availability; Total setup times; } \\
\text { Total job blocking time }\end{array}$ & & & & & & & & $\checkmark$ & & & $\checkmark$ & $\checkmark$ & $\checkmark$ & & \\
\hline Li et al. (2019b) & 2019 & 3 & & $\checkmark$ & & & Assembly; common due dates & & $\checkmark$ & SWT; $(\mathrm{TE}+\mathrm{TT})$ & & & & & & & & $\checkmark$ & & $\checkmark$ & & & $\checkmark$ & & \\
\hline Geng et al. (2019) & 2019 & $\mathrm{~m}$ & & $\checkmark$ & & & rcrc; on/off & & $\checkmark$ & $\begin{array}{c}\text { Cmax; Tmax; } \\
\text { Idle energy consumption }\end{array}$ & & & & & & & & $\checkmark$ & & & $\checkmark$ & & $\checkmark$ & & \\
\hline $\begin{array}{l}\text { Hao et al. (2019) } \\
\text { Zhou et al. (2019) }\end{array}$ & $\begin{array}{l}2019 \\
2019\end{array}$ & $\begin{array}{l}\mathrm{m} \\
2\end{array}$ & $\checkmark$ & $\begin{array}{l}\checkmark \\
v\end{array}$ & & & $\begin{array}{l}\text { Distributed } \\
\text { SIST; fmls; batch }\end{array}$ & $\begin{array}{l}v \\
v\end{array}$ & 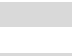 & $\begin{array}{l}\text { Cmax } \\
\text { TT }\end{array}$ & & & & & & & & $\checkmark$ & & $\checkmark$ & 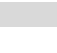 & & $\checkmark$ & & \\
\hline Xuan et al. (2019) & 2019 & $\mathrm{~m}$ & & & & $\checkmark$ & rj; SDST; step deteriorating job & $\checkmark$ & & TWCT & & & & & & & & $\checkmark$ & & $\checkmark$ & & & $\checkmark$ & & \\
\hline Han et al. (2019b) & 2019 & $\mathrm{~m}$ & & $\checkmark$ & & & $\begin{array}{l}\text { Block (limited buffer); } \\
\text { tj; public buffer }\end{array}$ & $\checkmark$ & & $\begin{array}{l}\text { Cmax; TMIT; Total plant factor; } \\
\text { Total workpiece blockage time }\end{array}$ & & & & & & & & $\checkmark$ & & $\checkmark$ & $\checkmark$ & $\checkmark$ & $\checkmark$ & & \\
\hline Zhang et al. (2019) & 2019 & $\mathrm{~m}$ & & $\checkmark$ & & - & SDST; variable speed levels & & $\checkmark$ & Cmax; TEC & & & & & & & & $\checkmark$ & & $\checkmark$ & & & $\checkmark$ & & \\
\hline Schulz et al. (2019) & 2019 & $\mathrm{~m}$ & & & & $\checkmark$ & & & $\checkmark$ & Cmax; TECC; Peak power & & $\checkmark$ & & & & & & $\checkmark$ & & $\checkmark$ & & & $\checkmark$ & & \\
\hline $\begin{array}{l}\text { Bozorgnezhad } \\
\text { et al. (2019) }\end{array}$ & 2019 & $\mathrm{~m}$ & & & & $\checkmark$ & SIST; worker assignment & $\checkmark$ & & Cmax & $\checkmark$ & & & & & & & $\checkmark$ & & $\checkmark$ & & & $\checkmark$ & & \\
\hline $\begin{array}{l}\text { Garavito-Hernández } \\
\text { et al. (2019) }\end{array}$ & 2019 & $\mathrm{~m}$ & & & & $\checkmark$ & SDST; MDST & $\checkmark$ & & Cmax & $\checkmark$ & & & & & & & $\checkmark$ & & $\checkmark$ & & & $\checkmark$ & & \\
\hline Raissi et al. (2019) & 2019 & $\mathrm{~m}$ & & $\checkmark$ & & & PM; budget constraint & $\checkmark$ & & Cmax & $\checkmark$ & & & & & & & $\checkmark$ & & $\checkmark$ & & & & $\checkmark$ & \\
\hline Zohali et al. (2019a) & 2019 & $\mathrm{~m}$ & & $\checkmark$ & & & SIST; lot sizing & $\checkmark$ & & $\mathrm{IHC}+\mathrm{SC}$ & $\checkmark$ & & & & & & & $\checkmark$ & & $\checkmark$ & & & $\checkmark$ & & \\
\hline Zohali et al. (2019b) & 2019 & $\mathrm{~m}$ & & $\checkmark$ & & & $\begin{array}{l}\text { SIST; lot sizing; } \\
\text { block (limited buffer) }\end{array}$ & $\checkmark$ & & $\mathrm{IHC}+\mathrm{SC}$ & $\checkmark$ & & & & & & & $\checkmark$ & & $\checkmark$ & & & $\checkmark$ & & \\
\hline $\begin{array}{l}\text { Chung and } \\
\text { Chen (2019) }\end{array}$ & 2019 & 2 & $\checkmark$ & $\checkmark$ & & & $\begin{array}{l}\text { SIST; lotstream; assembly; } \\
\text { distinct due windows }\end{array}$ & $\checkmark$ & & $\alpha(\mathrm{TE})+\beta(\mathrm{TT})$ & $\checkmark$ & & & & & & & $\checkmark$ & & $\checkmark$ & & & $\checkmark$ & & \\
\hline $\begin{array}{c}\text { Golneshini and } \\
\text { Fazlollahtabar (2019) }\end{array}$ & 2019 & $\mathrm{~m}$ & & & & $\checkmark$ & SIST; Mj; tj & $\checkmark$ & & $\mathrm{w}_{1}(\mathrm{TCT})+\mathrm{w}_{2}(\mathrm{Lmax})$ & $\checkmark$ & & & & & & & $\checkmark$ & & $\checkmark$ & & & & & $\checkmark$ \\
\hline $\begin{array}{c}\text { Rooeinfar } \\
\text { et al. }(2019) \\
\end{array}$ & 2019 & $\mathrm{~m}$ & & $\checkmark$ & & & Block (limited buffer); PM & $\checkmark$ & & TCT & $\checkmark$ & & & & & & & $\checkmark$ & & $\checkmark$ & & & & $\checkmark$ & \\
\hline
\end{tabular}




\begin{tabular}{|c|c|c|c|c|c|c|c|c|c|c|c|c|c|c|c|c|c|c|c|c|c|c|c|c|c|c|}
\hline \multirow{2}{*}{ Paper ID } & \multirow{2}{*}{ Year } & \multirow{2}{*}{$\begin{array}{l}\text { Stage } \\
\text { Type }\end{array}$} & \multirow{2}{*}{\multicolumn{4}{|c|}{$\begin{array}{c}\text { Parallel } \\
\text { Machines }\end{array}$}} & \multirow{2}{*}{$\begin{array}{l}\text { Contraint \& } \\
\text { Characteristics }\end{array}$} & \multirow{2}{*}{\multicolumn{2}{|c|}{$\begin{array}{c}\text { Type of } \\
\text { Objective }\end{array}$}} & \multirow{2}{*}{$\begin{array}{c}\text { Objective } \\
\text { Function(s) }\end{array}$} & \multicolumn{10}{|c|}{ Solution Algorithm (s) } & \multirow{2}{*}{\multicolumn{3}{|c|}{$\begin{array}{c}\text { Test } \\
\text { Instances }\end{array}$}} & \multicolumn{3}{|c|}{$\begin{array}{c}\text { Type of } \\
\text { Parameters }\end{array}$} \\
\hline & & & & & & & & & & & MIP & $\frac{\text { Exact }}{\text { Eps }}$ & Othr & CH & IH & RT & istic & Othr & $\begin{array}{c}\text { Meta- } \\
\text { Heuristic }\end{array}$ & Other & & $\mathbf{B P}$ & RCA & & & $\frac{F}{F}$ \\
\hline Oztop et al. (2019) & 2019 & $\mathrm{~m}$ & & $\checkmark$ & & & SIST; $\mathrm{tj}$ & $\checkmark$ & & TFT & $\checkmark$ & & & $\checkmark$ & & & & & $\checkmark$ & & $\checkmark$ & $\checkmark$ & & $\checkmark$ & & \\
\hline Mollaei et al. (2019) & 2019 & 3 & & & & $\checkmark$ & SDST; block (zero buffer); & & $\checkmark$ & $\begin{array}{c}\text { Cmax; Machine allocation } \\
\text { cost }\end{array}$ & & $\checkmark$ & & & & & & & & $\checkmark$ & $\checkmark$ & & & & & $\checkmark$ \\
\hline Liu et al. (2020b) & 2020 & $\mathrm{k}$ & & $\checkmark$ & & & On/off; multiple time factors & $\checkmark$ & & $\alpha \operatorname{Cmax}+(1-\alpha) \mathrm{TEC}$ & & & & & & & & & $\checkmark$ & & & & $\checkmark$ & $\checkmark$ & & \\
\hline Ding et al. (2020) & 2020 & $\mathrm{~m}$ & & $\checkmark$ & & & TOU; variable speed levels & & $\checkmark$ & TT; TECC & & $\checkmark$ & & & & & & & $\checkmark$ & & $\checkmark$ & & & $\checkmark$ & & \\
\hline $\begin{array}{l}\text { Marichelvam } \\
\text { et al. (2020) }\end{array}$ & 2020 & $\mathrm{~m}$ & & $\checkmark$ & & & $\mathrm{rj} ; \mathrm{LE} / \mathrm{FE}$ & $\checkmark$ & & $w_{1}(\mathrm{Cmax})+w_{2}(\mathrm{TFT})$ & & & & & & & & & $\checkmark$ & & $\checkmark$ & & $\checkmark$ & $\checkmark$ & & \\
\hline Oztop et al. (2020) & 2020 & $\mathrm{~m}$ & & $\checkmark$ & & & $\begin{array}{l}\text { SIST; tj; variable speed } \\
\text { levels }\end{array}$ & & $\checkmark$ & Cmax; TEC & & $\checkmark$ & & $\checkmark$ & & & & & $\checkmark$ & & $\checkmark$ & & & $\checkmark$ & & \\
\hline Li et al. (2020a) & 2020 & $\mathrm{~m}$ & & $\checkmark$ & & & SDST; distributed & $\checkmark$ & & Cmax & $\checkmark$ & & & & & & & & $\checkmark$ & & $\checkmark$ & & & $\checkmark$ & & \\
\hline Schulz et al. (2020) & 2020 & $\mathrm{~m}$ & & $\checkmark$ & & & TOU; variable speed levels & & $\checkmark$ & TT; TECC & & $\checkmark$ & & & & & & & & & $\checkmark$ & & & $\checkmark$ & & \\
\hline Cui et al. (2020) & 2020 & 3 & & $\checkmark$ & & & SIST; tj & $\checkmark$ & & $\begin{array}{l}\mathrm{TE}+\mathrm{TT}+\text { Prevention } \\
\text { temperature drop }\end{array}$ & & & & $\checkmark$ & & $\checkmark$ & & & & & $\checkmark$ & & & $\checkmark$ & & \\
\hline Geng et al. (2020a) & 2020 & $\mathrm{~m}$ & & & & $\checkmark$ & rerc; TOU & & $\checkmark$ & Cmax; TECC & & & & & & & & & $\checkmark$ & & & $\checkmark$ & & $\checkmark$ & & \\
\hline He et al. (2020) & 2020 & $\mathrm{k}$ & & & & $\checkmark$ & $\begin{array}{l}\text { SDST; t } \mathrm{j} \text {; lot sizing; } \\
\text { rush order insetion }\end{array}$ & & $\checkmark$ & $\begin{array}{l}\text { Cmax; Total transportation } \\
\text { time; } \\
\text { Total machine deviation }\end{array}$ & & & & & & & & & $\checkmark$ & & $\checkmark$ & & $\checkmark$ & $\checkmark$ & & \\
\hline Fu et al. (2020) & 2020 & $\mathrm{~m}$ & & & & $\checkmark$ & SIST; tj & & $\checkmark$ & $\begin{array}{l}\text { Cmax; Total noise pollution; } \\
\text { Total dust pollution }\end{array}$ & & & & & & & & & $\checkmark$ & & $\checkmark$ & & & $\checkmark$ & & \\
\hline Cao et al. (2020a) & 2020 & $\mathrm{~m}$ & & & & $\checkmark$ & & $\checkmark$ & & Cmax & & & & & & & & & $\checkmark$ & & & $\checkmark$ & $\checkmark$ & $\checkmark$ & & \\
\hline Kong et al. (2020) & 2020 & $\mathrm{k}$ & & & & $\checkmark$ & & $\checkmark$ & & $\alpha(\mathrm{Cmax})+\beta(\mathrm{TEC})+\gamma(\mathrm{PC})$ & & & & & & & & & $\checkmark$ & & $\checkmark$ & & & $\checkmark$ & & \\
\hline Cao et al. (2020b) & 2020 & 3 & & $\checkmark$ & & & $\begin{array}{l}\text { SIST; tj; limwait; TOU; } \\
\text { self generation cost; } \\
\text { on-grid electrovalence }\end{array}$ & & $\checkmark$ & Cmax; TECC & & & & & & & & & $\checkmark$ & & & & $\checkmark$ & $\checkmark$ & & \\
\hline Li et al. (2020b) & 2020 & $\mathrm{~m}$ & & & & & $\begin{array}{l}\text { Lotstream; due time window; } \\
\text { tj; variable sub-lots }\end{array}$ & & $\checkmark$ & AST; TEC; TE; TT & & & & & & & & & $\checkmark$ & & $\checkmark$ & $\checkmark$ & & $\checkmark$ & & \\
\hline Geng et al. (2020b) & 2020 & $\mathrm{~m}$ & & & & $\checkmark$ & Dual resource constraint & & $\checkmark$ & $\begin{array}{l}\text { Cmax; TT; Worker workload } \\
\text { balance }\end{array}$ & & & & & & & & & $\checkmark$ & & $\checkmark$ & & & $\checkmark$ & & \\
\hline Sun and Qi (2020) & 2020 & $\mathrm{k}$ & & & & $\checkmark$ & Customer requirements & $\checkmark$ & & Cmax + Delay cost & & & & & & & & & $\checkmark$ & & $\checkmark$ & & & $\checkmark$ & & \\
\hline Chen et al. (2020) & 2020 & $\mathrm{~m}$ & & & & $\checkmark$ & $\begin{array}{l}\text { rm; SDST; Mj; lotstream; } \\
\text { skip }\end{array}$ & & $\checkmark$ & Cmax; TEC & & & & & & & & & $\checkmark$ & & $\checkmark$ & & & $\checkmark$ & & \\
\hline Guo et al. (2020) & 2020 & 2 & & & & & SDST; batch & $\checkmark$ & & Cmax & $\checkmark$ & & & & & & & & $\checkmark$ & & $\checkmark$ & & & $\checkmark$ & & \\
\hline Gong et al. (2020) & 2020 & $\mathrm{~m}$ & & & & $\checkmark$ & Worker flexibility & & $\checkmark$ & $\begin{array}{c}\text { Cmax; WC; } \\
\text { Green production indicator }\end{array}$ & $\checkmark$ & & & & & & & & $\checkmark$ & & & $\checkmark$ & & $\checkmark$ & & \\
\hline $\begin{array}{l}\text { Baxendale } \\
\text { et al. (2020) }\end{array}$ & 2020 & 2 & $\checkmark$ & $\checkmark$ & & & $\begin{array}{c}\text { Batch; Mj; } \\
\text { work shift constraint }\end{array}$ & $\checkmark$ & & TT & $\checkmark$ & & & & & & & & $\checkmark$ & & $\checkmark$ & & & $\checkmark$ & & \\
\hline Lei et al. (2020) & 2020 & $\mathrm{k}$ & & & & $\checkmark$ & $\begin{array}{l}\text { Block (zero buffer); dynamic } \\
\text { transport waiting times }\end{array}$ & $\checkmark$ & & Cmax & $\checkmark$ & & & & & & & & $\checkmark$ & & $\checkmark$ & & & $\checkmark$ & & \\
\hline Costa et al. (2020) & 2020 & $\mathrm{~m}$ & & $\checkmark$ & & & SIST; workforce constraint & $\checkmark$ & & Cmax & $\checkmark$ & & & & & & & & $\checkmark$ & & $\checkmark$ & & & $\checkmark$ & & \\
\hline Hasani et al. (2020) & 2020 & $\mathrm{k}$ & $\checkmark$ & & & $\checkmark$ & $\begin{array}{c}\text { SDST; skip; machine } \\
\text { dependent processing stages }\end{array}$ & & $\checkmark$ & TEC; PC & & $\checkmark$ & & & & & & & $\checkmark$ & & $\checkmark$ & & & $\checkmark$ & & \\
\hline Li et al. (2020c) & 2020 & $\mathrm{~m}$ & & & & $\checkmark$ & SDST; distributed & $\checkmark$ & & Cmax & $\checkmark$ & & & & & & & & $\checkmark$ & & $\checkmark$ & & & $\checkmark$ & & \\
\hline Shao et al. (2020) & 2020 & $\mathrm{~m}$ & & $\checkmark$ & & & skip; distributed & $\checkmark$ & & Cmax & & & & $\checkmark$ & & & & & $\checkmark$ & & $\checkmark$ & & & $\checkmark$ & & \\
\hline Wang et al. (2020) & 2020 & 2 & $\checkmark$ & $\checkmark$ & & & Batch; Mj; TOU; on/off & & $\checkmark$ & Cmax; TEC & & $\checkmark$ & & $\checkmark$ & $\checkmark$ & & & & $\checkmark$ & & $\checkmark$ & & $\checkmark$ & $\checkmark$ & & \\
\hline Liu et al. (2020c) & 2020 & 3 & $\checkmark$ & & & $\checkmark$ & $\begin{array}{c}\text { SIST; batch; Mj; TOU; } \\
\text { on/off }\end{array}$ & & $\checkmark$ & Cmax; TECC & & $\checkmark$ & & $\checkmark$ & & & & & $\checkmark$ & & $\checkmark$ & & & & & \\
\hline
\end{tabular}




\section{Literature Analysis}

In this section, the statistical and mathematical analyses related to included HFS articles have been realized according to various characteristics such as year, country, journal, number of stages, type of parallel machines, constraints, objective functions, solution methods, test instances and type of parameters. Different types of charts have been utilized for visual presentation of analysis results. Moreover, it has been aimed to answer 14 predetermined research questions through statistical analyses and present a road map for researchers studied in this field with respect to recent developments and future research opportunities.

RQ1: How is the distribution of HFS studies according to years?

The number of HFS articles according to years for the 2010-2020 interval have been presented in Fig. 3. As seen in this figure, as well as the most publications made in the years 2019 and 2020, this subject has remained valid and the number of publications related to it over the years. Therefore, it is possible to say that HFS is a worth-studying type of scheduling problems and it is a significant field in the scheduling literature.

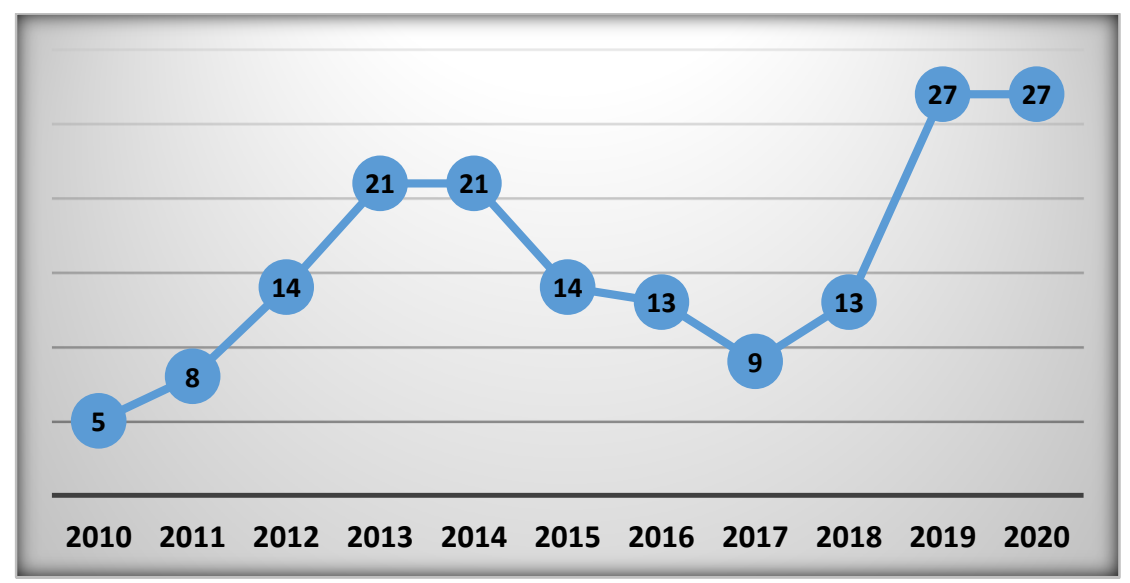

Fig. 3. Number of HFS articles according to years

RQ2: How is the distribution of HFS studies according to countries?

The percentage distribution of HFS articles according to countries has been given in Figure 4. As seen in this figure, the HFS subject is studied in many countries and the most publications have been made in China and Iran with the percentages of 34 and 21 respectively. When it is considered that HFS is studied in 29 different countries around the world, it is possible to say that this subject is valid and well-known in the scheduling field around the world.

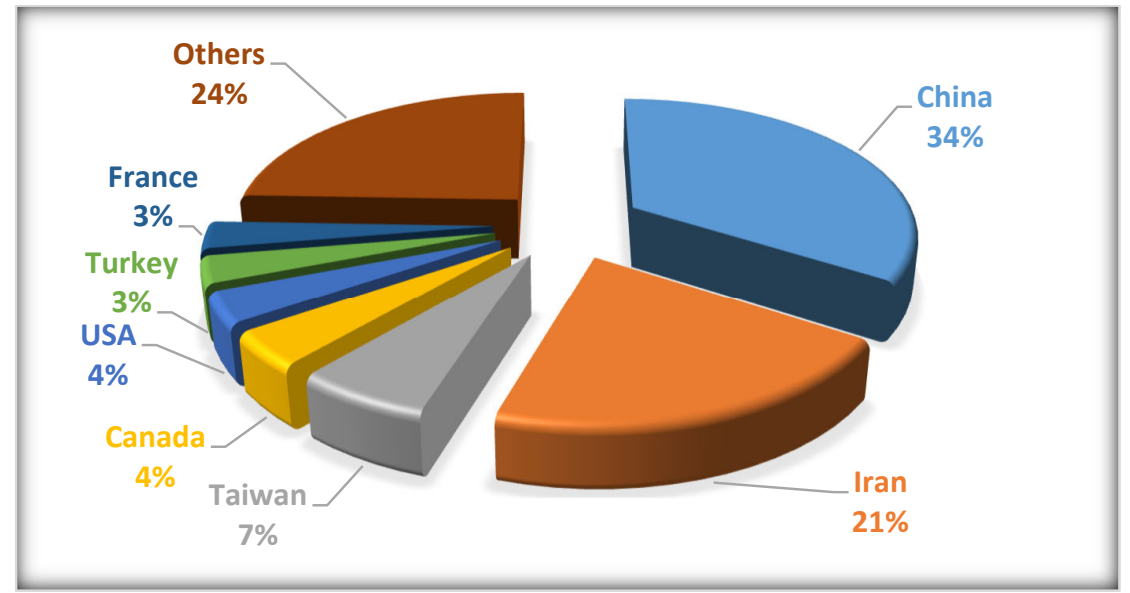

Fig.e 4. Percentage distribution of HFS articles according to countries 
RQ3: In which journals HFS articles are published?

The analysis results of HFS articles according to journals have been presented in Figs. 5-6. As seen in Fig. 5, International Journal of Production Research has the most articles related to HFS with the percentage of 18. International Journal of Advanced Manufacturing Technology, Computers \& Industrial Engineering, Applied Soft Computing and Computers \& Operations Research journals follow it with close percentages to each other. Although HFS articles have been published in 55 different journals according to analysis results, it can be inferred that the first five journals have more possibility to make a publication than the others related to this subject. As seen in Figure 6, 89\% of these journals are in the Q1 (61\%) and Q2 (28\%) according to Scimago Journal and Country Rank (SJR) 2019 data. Besides, 97\% of HFS articles have been published in Q1 (82\%) and Q2 (15\%) journals. Therefore, it is possible to say that this subject has a high possibility of being published in a high-ranked journal. Besides, when the journals are analyzed according to subject areas it is seen that engineering (industrial and manufacturing, chemical, mechanical et al.), computer science, and artificial intelligence are located in the first three orders. This information can be beneficial for researchers in the journal selection process for HFS articles.

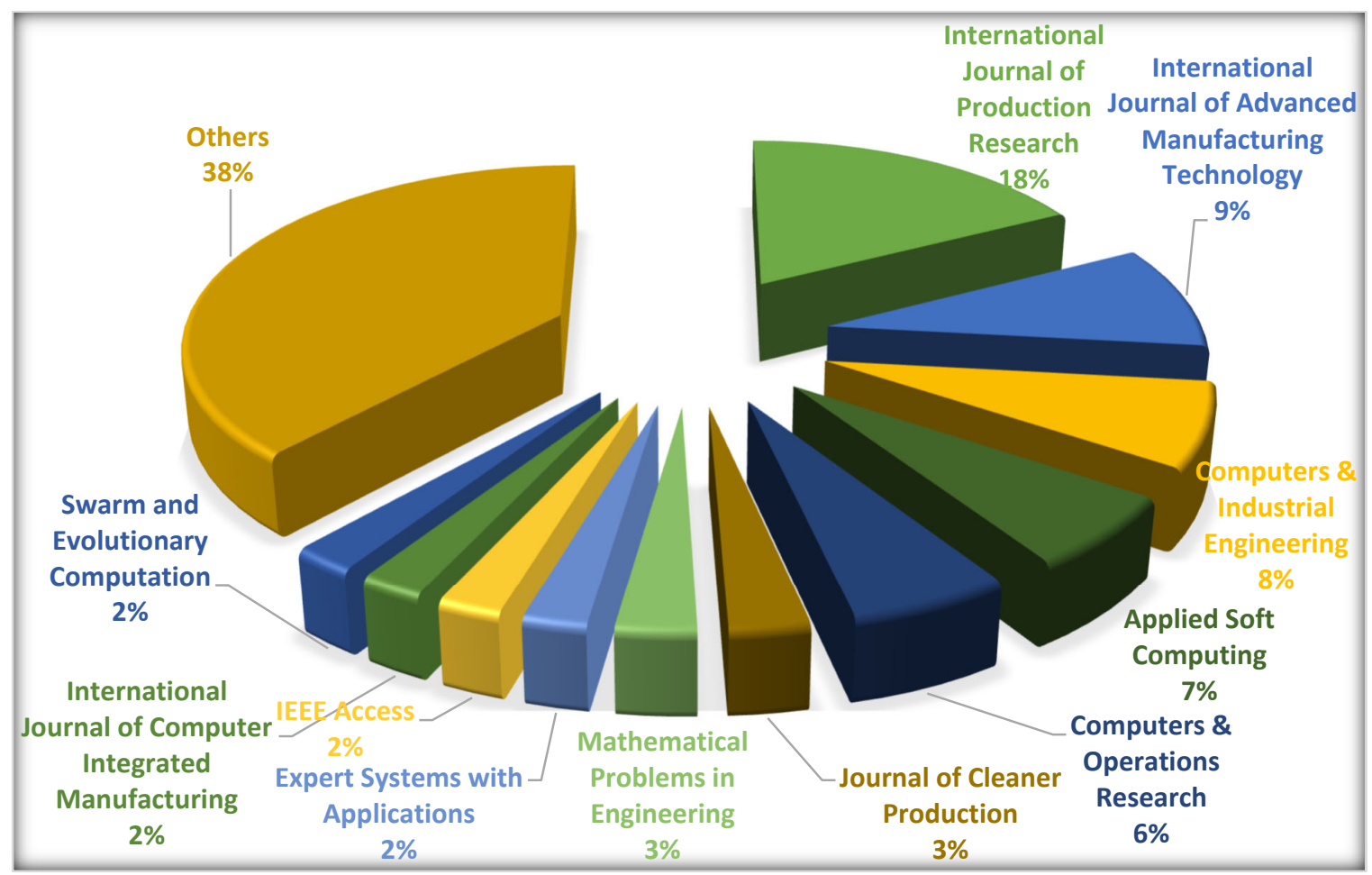

Fig. 5. Percentage distribution of HFS articles according to journals

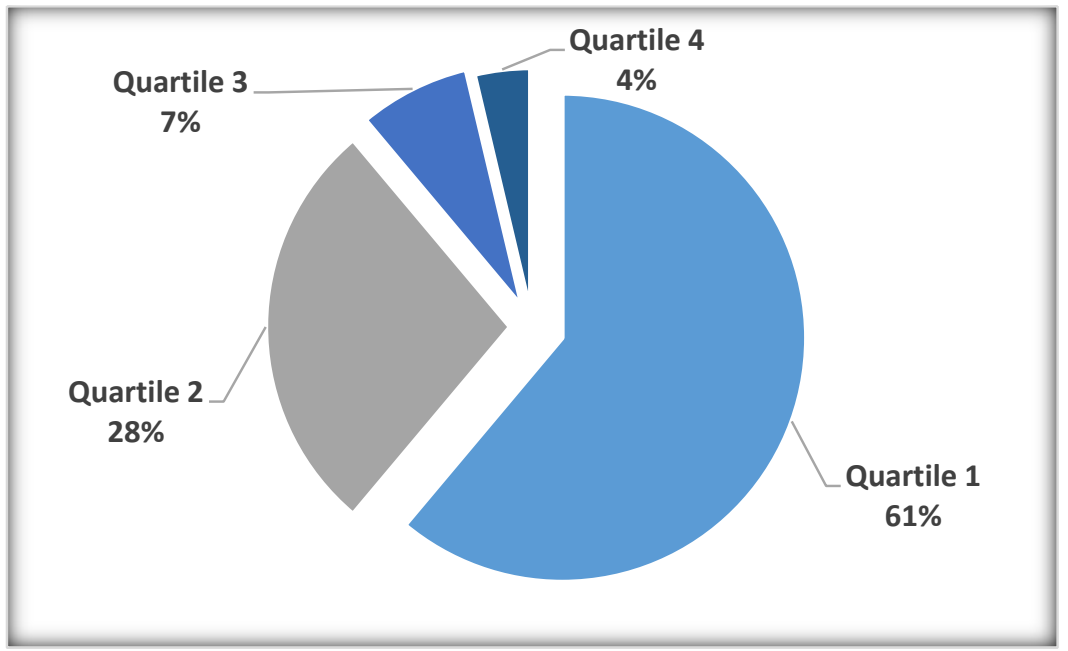

Fig. 6. Percentage distribution of journals according to SJR 2019 quartile data 
RQ4: How is the distribution of HFS studies according to the number of stages?

We classified HFS articles according to the number of stages as 2-stage, 3-stage, $\mathrm{k}$-stage $(\mathrm{k}>3)$ and m-stage (different stage numbers utilized for test instances) and the percentage distribution of them has been given in Fig. 7. As seen in this figure, $\mathrm{m}-$ stage is the most utilized stage number in HFS articles due to mostly usage of RGTPs and BPs for computational experiments. Besides, 2-stage, k-stage and 3-stage HFSPs follow it respectively.

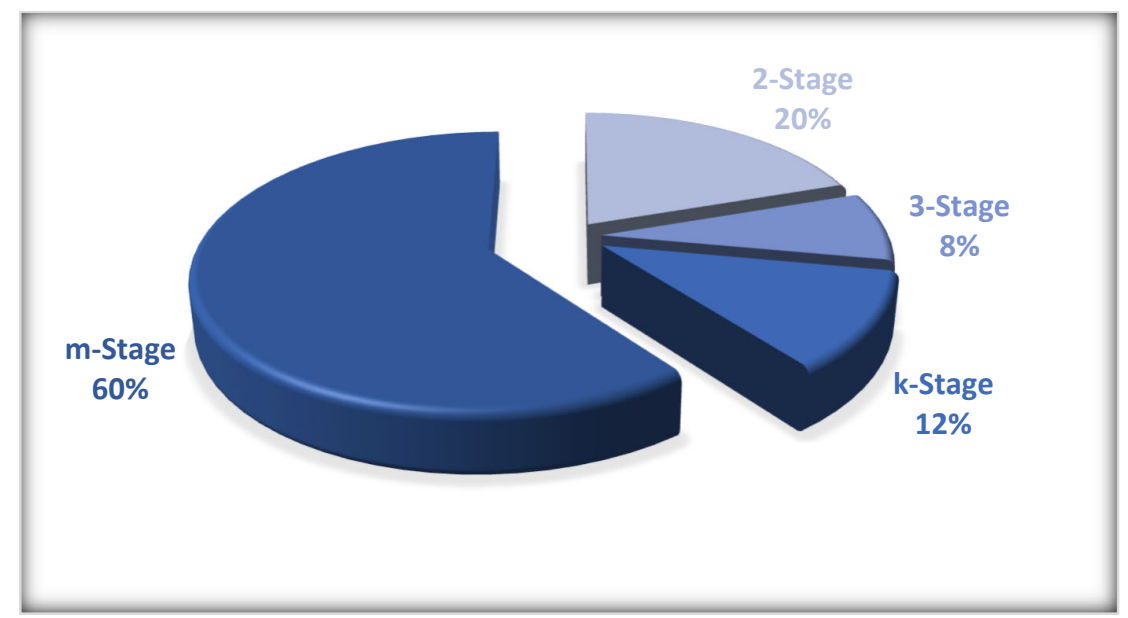

Fig. 7. Percentage distribution of HFS articles according to number of stages

RQ5: Which types of parallel machines are utilized in each stage?

The percentage distribution of HFS articles according to the type of parallel machines has been presented in Fig. 8. As seen in this figure, identical machines are the most utilized machines with the percentage of 66 and unrelated and uniform machines follow it respectively. It is possible to say that, while identical machines are mostly utilized for theoretical problems, unrelated machines are utilized as more realistic characteristics for HFSPs. A single machine has been utilized in one of the stages in the $12 \%$ of HFS articles.

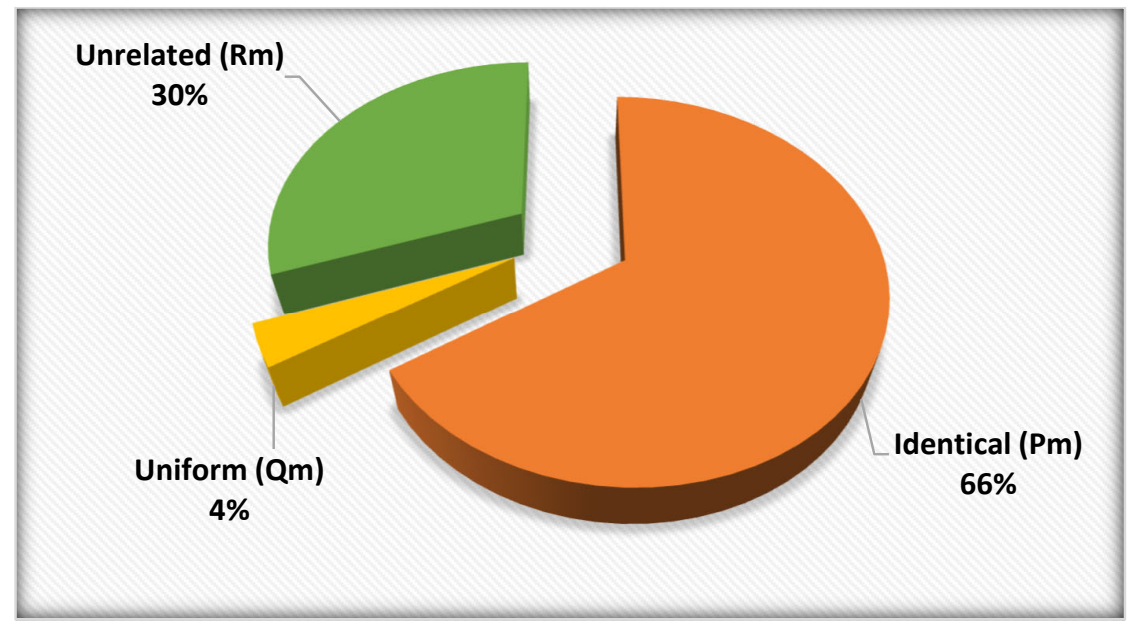

Fig. 8. Percentage distribution of HFS articles according to type of parallel machines

RQ6: Which constraints are considered in HFSPs?

The analysis results of HFS articles according to constraints have been given in Figs. 9-10. While the constraints have been analyzed individually in Fig. 9, all constraints considered in HFS articles have been collected under 5 main topics as jobrelated, machine-related, energy-related, worker-related, and others in Figure 10. SDSTs are the most considered constraints in HFSPs and it is followed by SISTs, batch processing, stage skipping, and machine eligibility. While these constraints compose the first 5 constraints, transportation times, release dates, blocking, lot streaming, job families, and recirculation are also mostly utilized constraints. As seen in Fig. 10, job-related constraints have the highest percentage as $75 \%$ in HFS articles 
and machine-related, energy-related, and worker-related constraints follow them respectively. Due to the increasing importance of the energy-efficient scheduling concept, energy-related constraints such as TOU electricity prices, turn on/off strategy and variable speed levels have been more considered in HFSPs in recent years as also seen in Table 3.

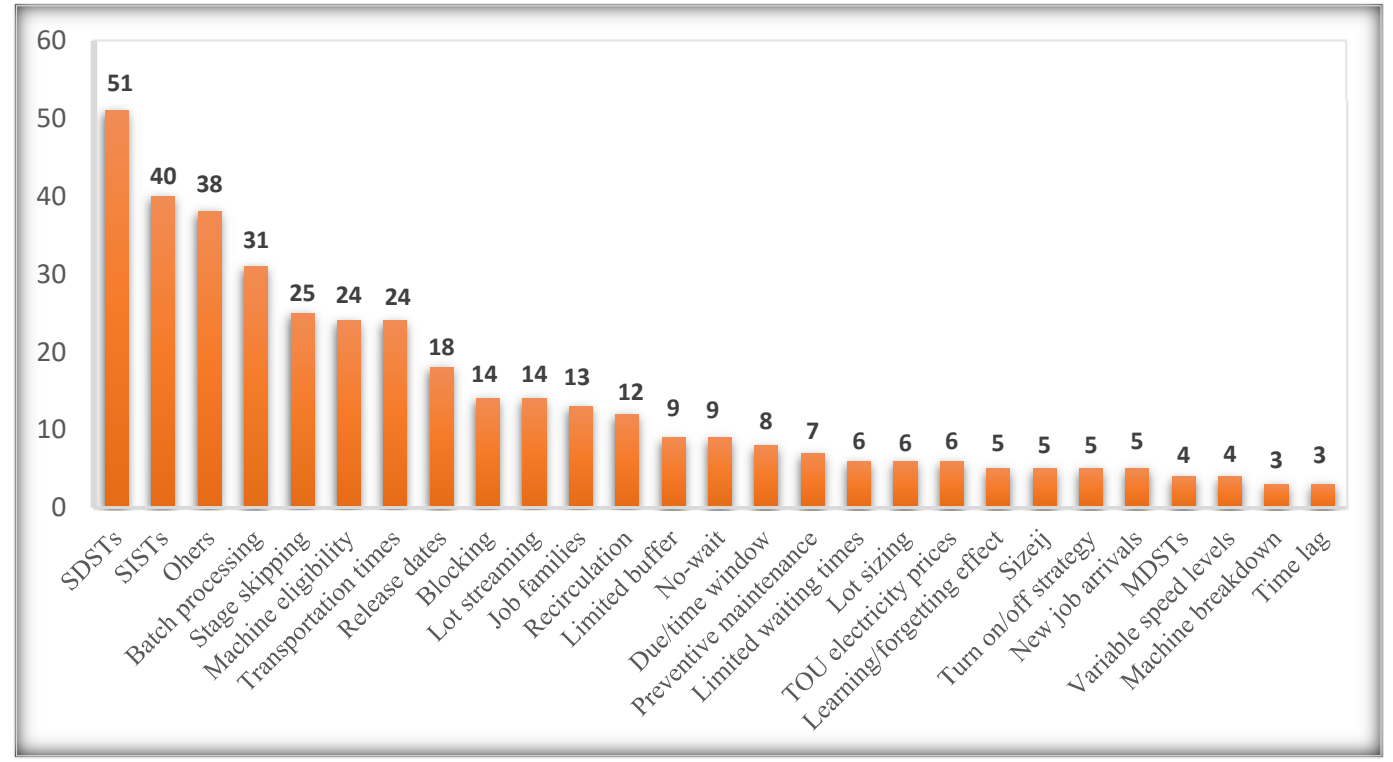

Fig. 9. Numerical analysis of constraints utilized in HFS articles

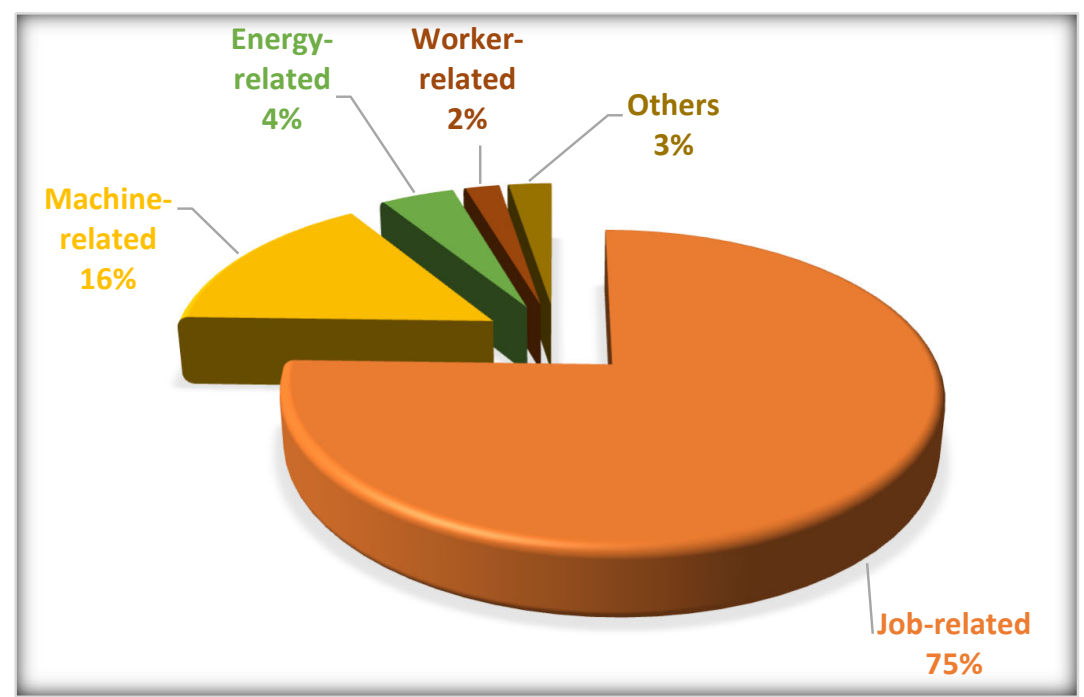

Fig. 10. A classification for constraints of HFSPs

RQ7: Which objective functions are aimed to optimize in HFSPs?

The numbers of HFS articles according to years based on type of objective functions have been given in Table 4. Furthermore, the analysis results for HFS articles related to objective functions have been presented in Figs. 11-14. It is possible to classify scheduling problems as single-objective and multi-objective in terms of the number of objective functions aimed to optimize. Multi-objective problems can be converted to a single-objective problem by using normal and weighted sum of different objectives in order to solve them easier. Therefore, we classified articles as multi-objective and single-objective (monoobjective which has only one objective, normal sum of different objectives or weighted sum of different objectives). As seen in Figure 11, mono-objective HFSPs are the most studied problems with the percentage of 55. It is obvious that authors prefer normal sum $(7 \%)$ or weighted sum $(17 \%)$ of different objectives to convert multi-objective scheduling problems to singleobjective and thus solve them more easily. When the objectives are evaluated individually it is seen that makespan which is a significant indicator of effective system utilization is the most utilized objective function with the percentage of 37 as shown in Fig. 12. Total tardiness and total earliness follow this objective with the percentages of 13 and 8 respectively as due-date 
based criteria. As seen in Fig. 13, completion-time based criteria related to system utilization are the most utilized objectives with the percentage of $44 \%$ and due date-based criteria related to customer satisfaction are in the second order with the percentage of 26. Besides, owing to the increasing importance of a cleaner production concept, environment-related criteria such as total energy consumption, total energy consumption cost, and green production indicator have been more considered in recent years as also seen in Table 3. As seen in Fig. 14, the studies which handle more than one objective have increased in recent years. In this context, we can say that multidimensional problems can be more acceptable.

\section{Table 4}

Number of HFS articles according to years based on type of objective functions

\begin{tabular}{|c|c|c|c|}
\hline Year & $\begin{array}{c}\text { Mono- } \\
\text { Objective }\end{array}$ & $\begin{array}{c}\text { Normal Sum of } \\
\text { Different Objectives }\end{array}$ & $\begin{array}{c}\text { Weighted Sum of } \\
\text { Different Objectives }\end{array}$ \\
\hline 2010 & 3 & 1 & 0 \\
\hline 2011 & 3 & 1 & 2 \\
\hline 2012 & 11 & 0 & 2 \\
\hline 2013 & 14 & 1 & 5 \\
\hline 2014 & 13 & 2 & 3 \\
\hline 2015 & 8 & 1 & 4 \\
\hline 2016 & 6 & 0 & 3 \\
\hline 2017 & 6 & 2 & 1 \\
\hline 2018 & 7 & 0 & 4 \\
\hline 2019 & 15 & 2 & 3 \\
\hline 2020 & 8 & 2 & 3 \\
\hline \multirow[t]{2}{*}{ Total } & 94 & 12 & 30 \\
\hline & $\begin{array}{c}\text { Multi- } \\
\text { objective; } \\
36 ; 21 \%\end{array}$ & $\begin{array}{r}\text { Si } \\
\text { obj } \\
136\end{array}$ & $\begin{array}{l}\text { Weighted sum } \\
\text { of different } \\
\text { objectives; } \\
30 ; 17 \%\end{array}$ \\
\hline
\end{tabular}

Fig. 11. Percentage distribution of HFS articles according to type of objectives

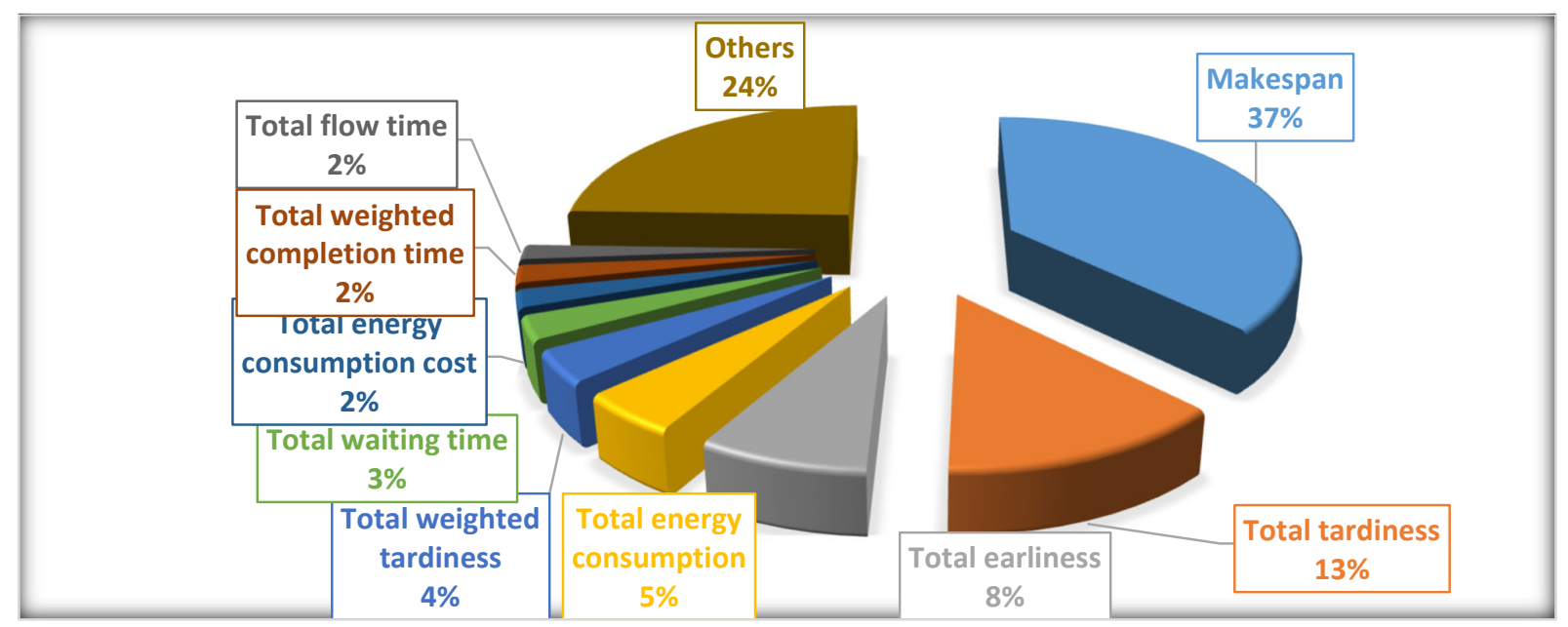

Fig. 12. Percentage distribution of objective functions for HFSPs 


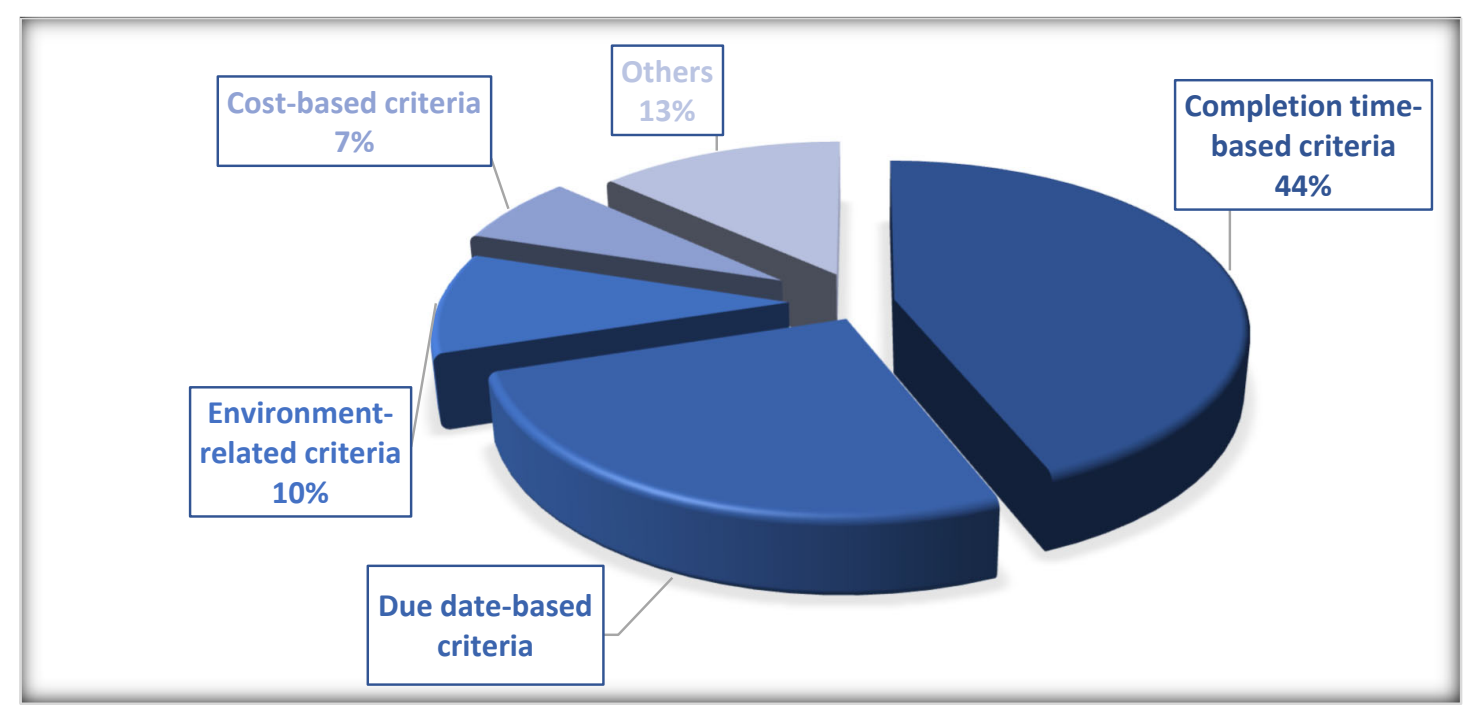

Fig. 13. A classification for objectives of HFSPs

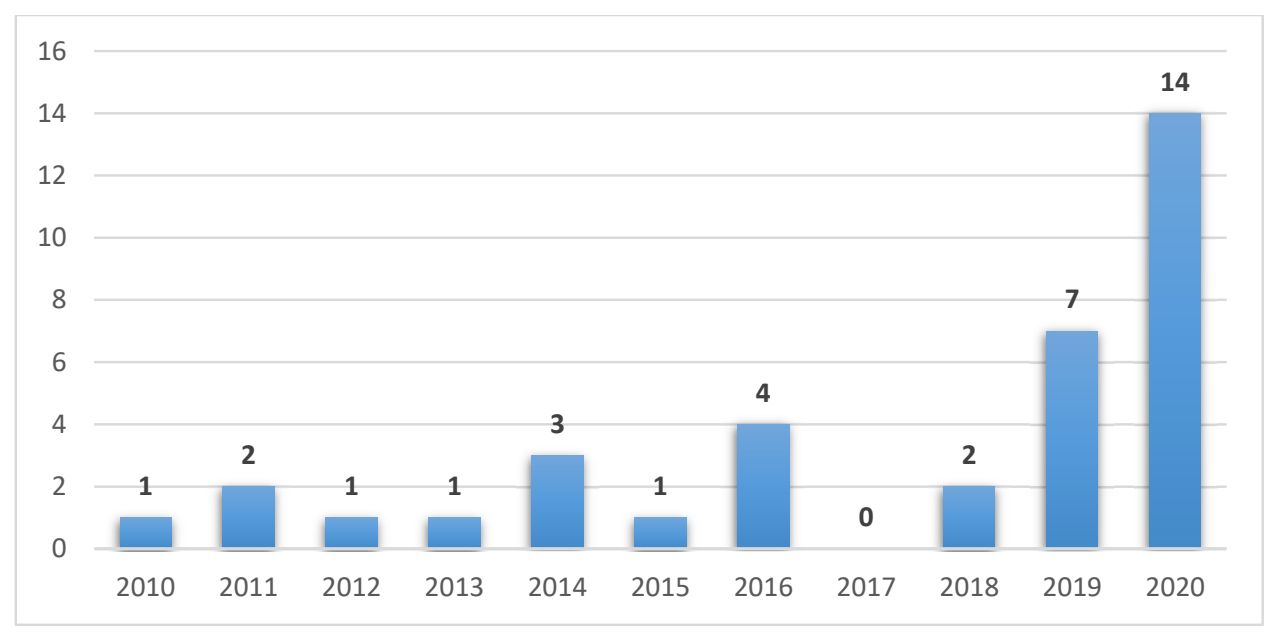

Fig. 14. Number of multi-objective HFS articles according to years

RQ8: Which solution methods are utilized to solve HFSPs?

Since HFSP is located in NP-hard class, exact algorithms are able to solve only small instances because of computational complexity. Therefore, heuristic and metaheuristic algorithms are effectively utilized to obtain near optimal solutions for large sizes of HFSPs in a reasonable time. In the analysis of solution algorithms utilized to deal with HFSP, we divided HFS articles into 8 main topics as exact, heuristic, metaheuristic, exact+heuristic, exact + metaheuristic, heuristic + metaheuristic, exact+heuristic+metaheuristic and other methods in order to provide a more detailed classification for researchers. The number of HFS articles according to years based on solution algorithms have been given in Table 5. Moreover, the result of classification based on solution algorithms has been presented in Figure 15 and it is seen that metaheuristics have been applied in $84 \%$ of HFS articles as single or hybrid due to effectiveness and performance of them to solve large size problems. As seen in Figure 16, the percentages of metaheuristics, exact algorithms, heuristics, and other methods can be given as 55\%, 32\%, $12 \%$ and $1 \%$ respectively by considering solution algorithms individually. It is also seen in this figure that metaheuristics are mostly used and exact algorithms, heuristics and other methods follow these algorithms respectively. While MIP models solved by a suitable solver are utilized to obtain exact solutions for small-size single-objective HFSPs, the $\varepsilon$-constraint method is applied for small instances of multi-objective problems. We analyzed heuristic algorithms under 5 topics as constructive heuristic $(\mathrm{CH})$, improvement heuristic (IH), relaxation technique (RT), decomposition based approach (DBA), and others and it is seen that constructive heuristics are mostly utilized as also seen in Table 3. 
Table 5

Number of HFS articles according to years based on solution algorithms

\begin{tabular}{|c|c|c|c|c|c|c|}
\hline Year & Exact & Heuristic & Metaheuristic & Other & $\begin{array}{c}\text { Single } \\
\text { Metaheuristic }\end{array}$ & $\begin{array}{c}\text { Hybrid } \\
\text { Metaheuristic }\end{array}$ \\
\hline 2010 & 2 & 2 & 3 & 0 & 1 & 2 \\
\hline 2011 & 2 & 1 & 7 & 0 & 3 & 5 \\
\hline 2012 & 8 & 2 & 9 & 0 & 5 & 4 \\
\hline 2013 & 9 & 2 & 17 & 1 & 8 & 9 \\
\hline 2014 & 10 & 4 & 18 & 0 & 8 & 10 \\
\hline 2015 & 9 & 3 & 10 & 1 & 5 & 5 \\
\hline 2016 & 7 & 2 & 13 & 0 & 8 & 5 \\
\hline 2017 & 5 & 6 & 5 & 0 & 1 & 3 \\
\hline 2018 & 6 & 2 & 11 & 0 & 3 & 8 \\
\hline 2019 & 13 & 1 & 26 & 1 & 5 & 20 \\
\hline 2020 & 13 & 5 & 25 & 0 & 10 & 16 \\
\hline Total & 84 & 30 & 144 & 3 & 57 & 87 \\
\hline
\end{tabular}

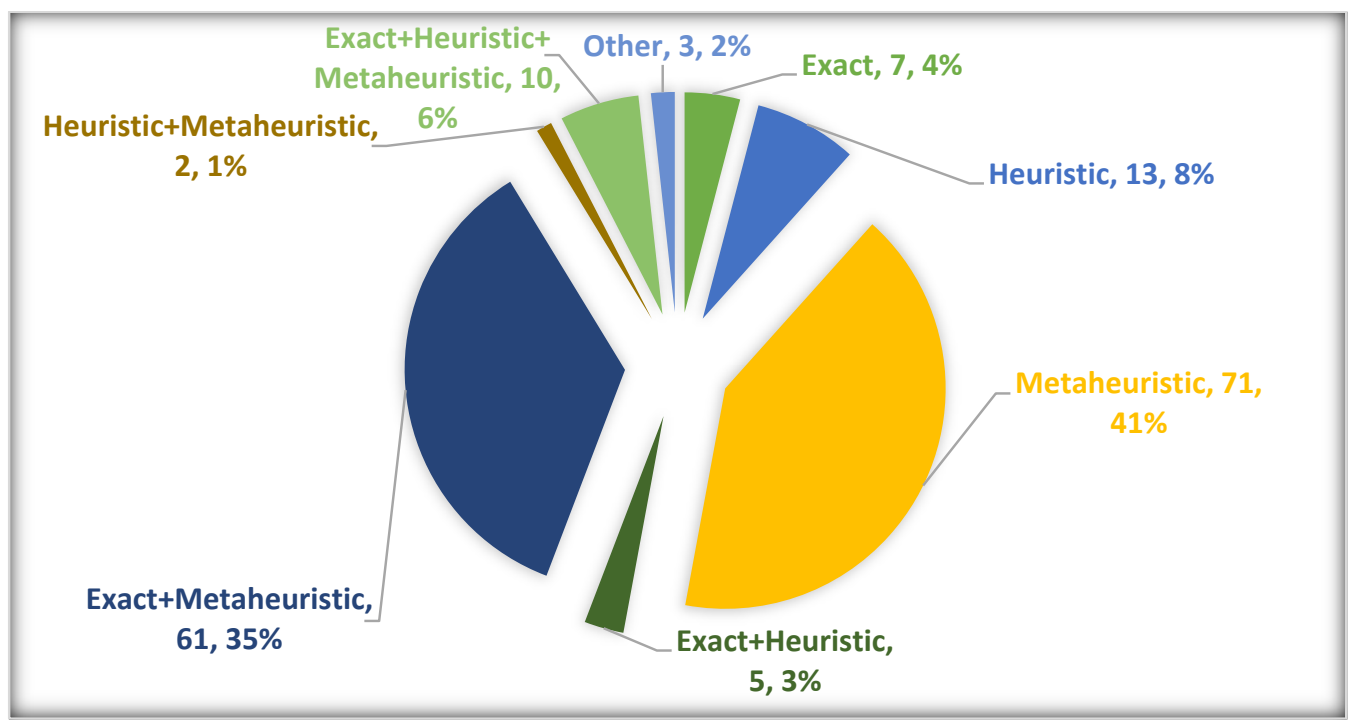

Fig. 15. Percentage distribution of HFS articles according to solution algorithms

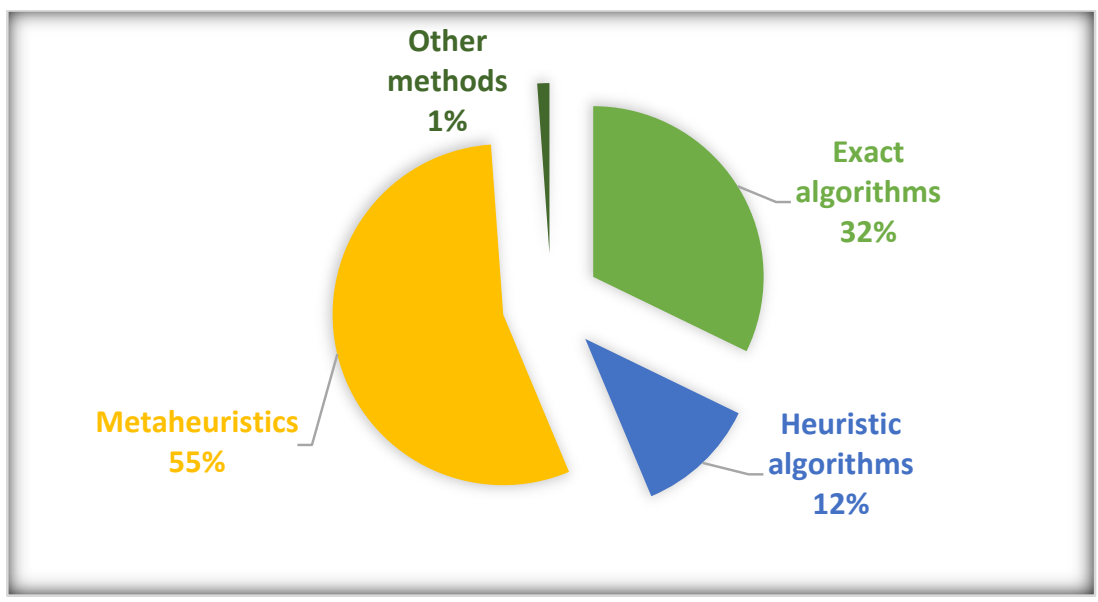

Fig. 16. Percentage distribution of solution algorithms for included HFS articles 
RQ9: Which metaheuristic algorithms are utilized to obtain near optimal solutions in HFSPs?

As we mentioned previously metaheuristics are widely utilized for HFSPs and the analysis results related to metaheuristics have been given in Fig. 17 separately. As seen in this figure, if we consider all algorithms individually GA, SA and PSO are the most utilized metaheuristics for HFSPs. In addition to this, NSGA-II is the most common metaheuristic to cope with multiobjective problems. It can also be said that, a wide range of metaheuristics which have different solution procedures are located in the literature except existing in this figure and others topic includes them. We can ensample these metaheuristics as AIS, CS, MBO, SFLA, TLBO, WFA, and SPEA-II. It is possible to say that, metaheuristics are utilized as single or hybrid to generate solutions. Hybrid metaheuristics which integrate metaheuristics with metaheuristic, heuristic or other methods are applied as novel and effective approaches to improve solution quality. As seen in Fig. 18, the usage of hybrid metaheuristics increases in recent years.

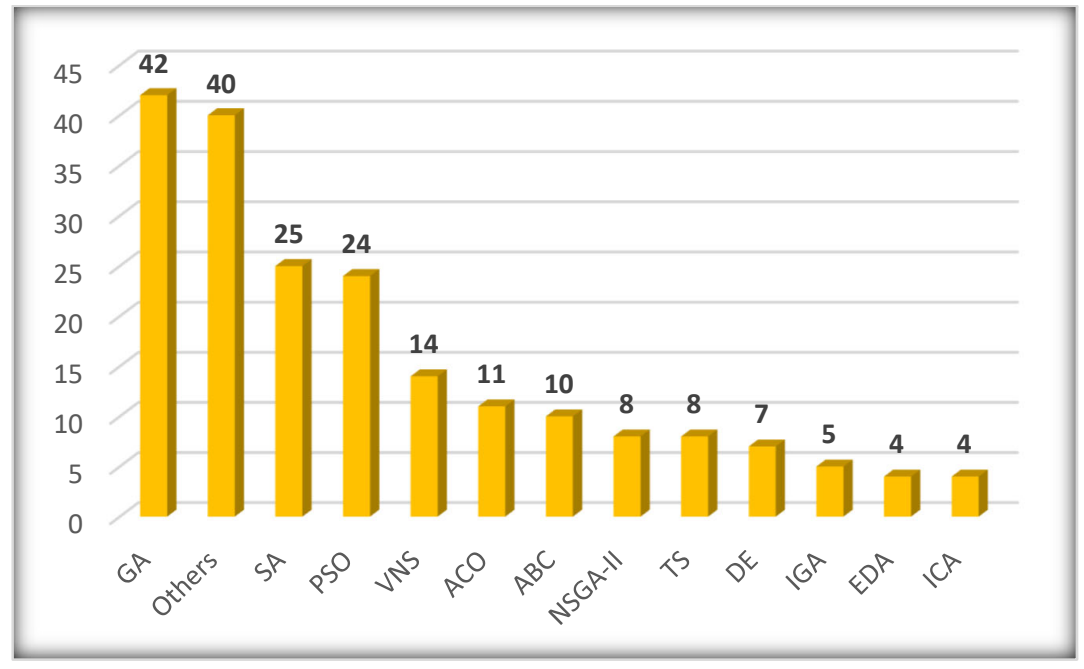

Fig. 17. Numerical analysis of metaheuristics utilized for HFSPs

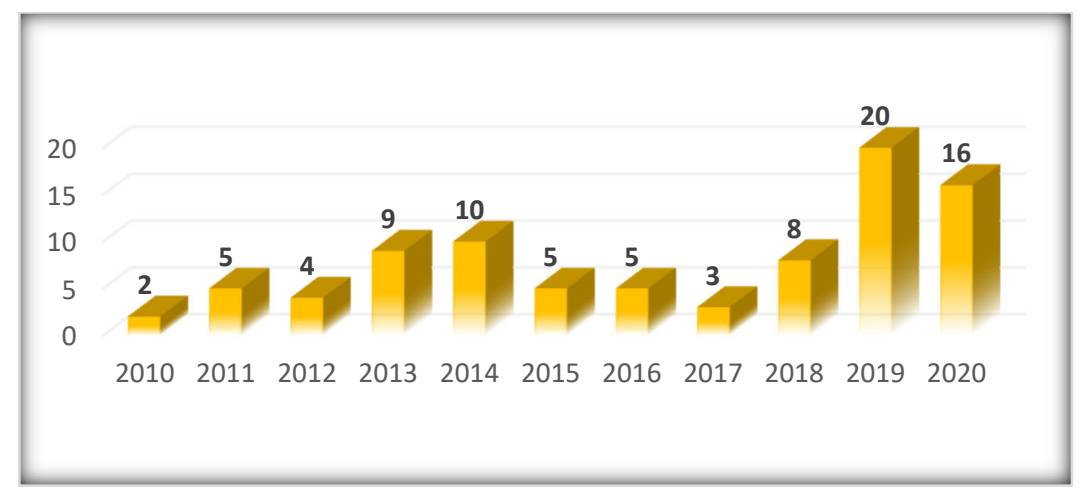

Fig. 18. Numbers of hybrid metaheuristics according to years

RQ10: Which type of test instances are utilized to analyze performance of the proposed methods in HFSPs?

RQ11: Which manufacturing applications are realized as HFSP in real life?

We classified test instances utilized for HFSPs as RGTPs, BPs and RCAs and percentage distribution of HFS articles according to these topics has been presented in Fig. 19. When there is no benchmark for a problem in the literature, test problems are generated randomly according to parameters of the problem. As seen in Fig. 19, RGTPs are the most utilized test instances for HFSPs with the percentage of 74. The percentage of RCA is 15 and it is possible to say that this problem is significant in real-life according to this statistic. Steelmaking continuous casting, solar cell manufacturing, furniture manufacturing, printed circuit board manufacturing, bus manufacturing, seamless steel tube production, tile manufacturing and cast steel valve manufacturing can be given as real-life HFSP examples. 


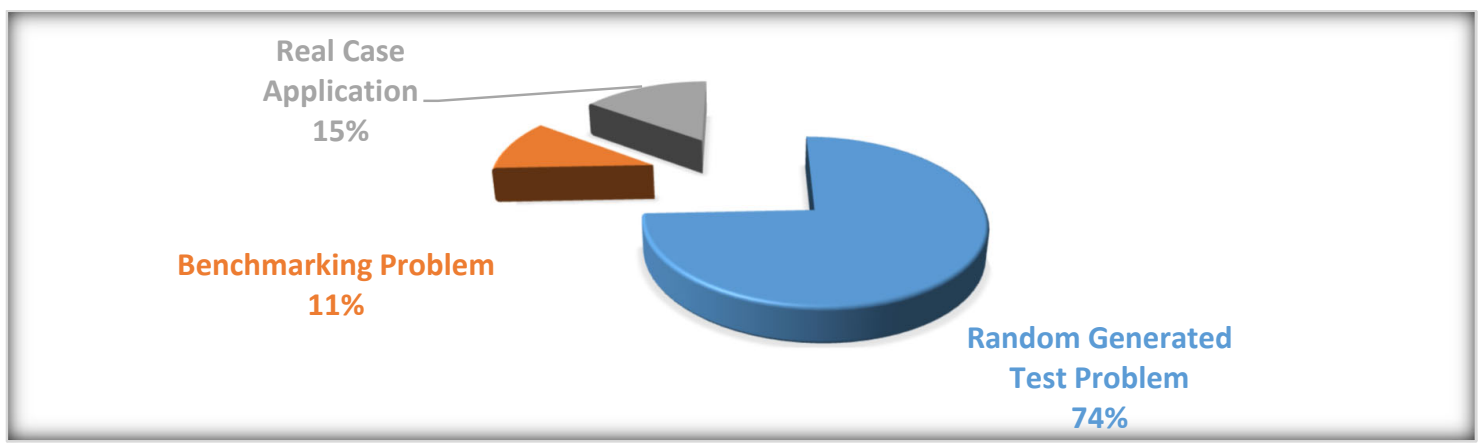

Fig. 19. Percentage distribution of HFS articles according to test instances

RQ12: In which industries HFS applications are realized in manufacturing?

It is possible to say that many HFS problems related to different industries are handled in the literature. Percentage distribution of real-life HFSPs according to industries has been presented in Fig. 20. As seen in this figure, steel, electronic and automotive are leading industries with the percentages of 25,23 , and 17 respectively. Others include different industries such as aerospace, bio-process, energy, paper, and shipbuilding industries.

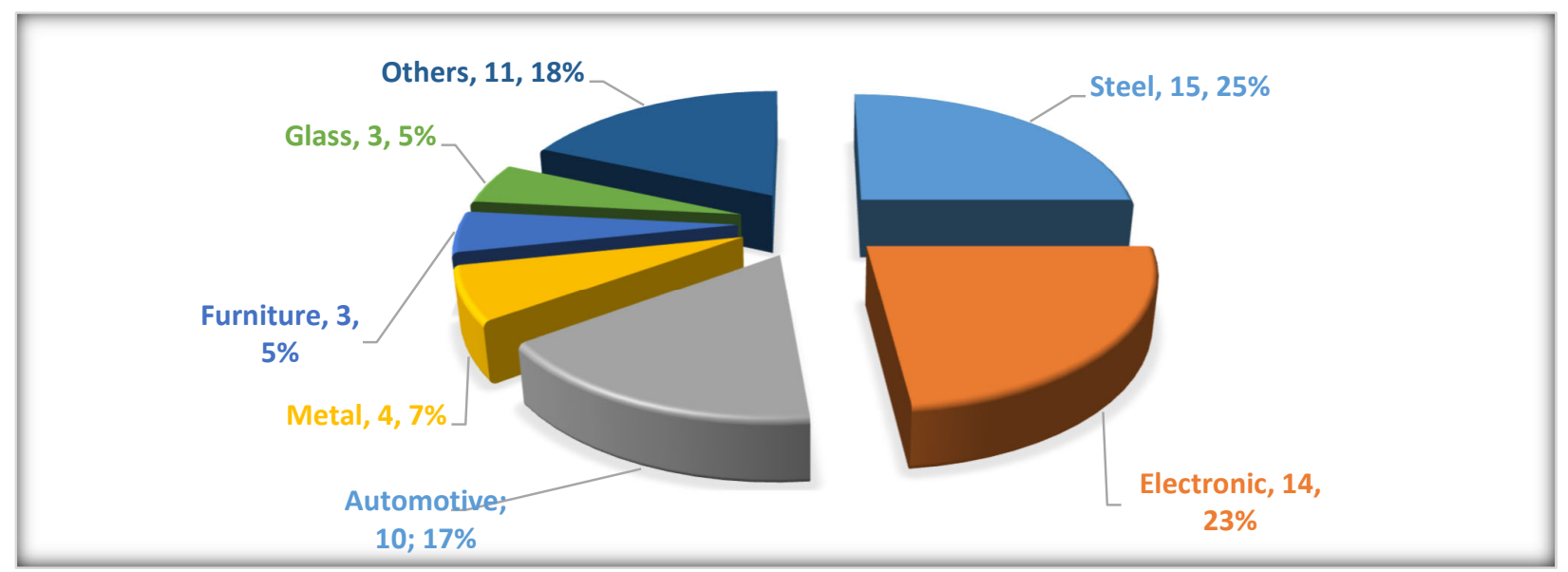

Fig. 20. Percentage distribution of HFS manufacturing problems according to industries

RQ13: Which types of parameters (deterministic, stochastic or fuzzy) are utilized in HFSPs?

RQ14: Which stochastic or fuzzy parameters are utilized in HFSPs?

The percentage distribution of HFS articles according to parameter types has been given in Fig. 21. As seen in this figure, the highest percentage $(94 \%)$ of HFS articles utilize deterministic parameters. The percentages of stochastic and fuzzy parameters are 4 and 2 respectively. Processing times, setup times and due dates are utilized as stochastic or fuzzy parameters in HFSPs to cope with uncertainties in the manufacturing environment.

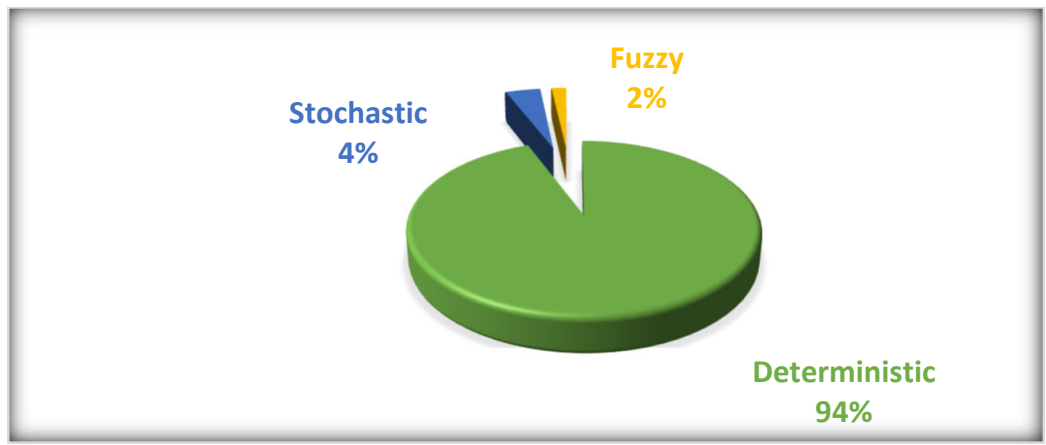

Fig. 21. Percentage distribution of HFS articles according to type of parameters 


\section{Conclusion and Future Research Suggestions}

HFSP which is a combination of PMS and classical FS problems includes more than one stage that at least one of these stages has identical, uniform or unrelated parallel machines and it is aimed to assign jobs to parallel machines at each stage and to sequence jobs assigned to each machine in this problem. There are different areas that HFS is applied to such as manufacturing, healthcare management, transportation, cloud computing and agriculture and many studies have been realized over the years related to this subject in the literature. Therefore, in this paper, we aimed to present a more systematic, extensive and updated literature survey than existing review studies in the literature with respect to HFS through PRISMA methodology which enables to reveal a systematic review for a specified subject. We utilized several exclusion criteria to eliminate some papers reached as a result of database searching with different keywords and to provide a focused paper. At the end of the methodological review process, 172 HFS research articles which are related to production scheduling, are published in the 2010-2020 year interval, and utilize a mathematical model to clarify HFSP. Then, these articles have been analyzed according to some features such as year, country, journal, stage type, parallel machines, constraints, objective functions, solution methods, test instances and parameter types and the results of analyses have been presented. Thus, it has been desired to present recent developments related to this subject and provide a beneficial road map to researchers who study in this field.

As a result of this literature review, we saw that HFS has remained valid over the years and we inferred that it will be studied in the next few years from the number of articles according to years. We determined that the included articles are from 29 different countries and are published in 55 different mostly high-ranked journals.

It has been seen that m-stage is the most common type in terms of stage type and we thought that this situation is rooted from more usage of RGTPs and BPs. It means that HFSP is solved for different stage numbers instead of only one constant stage. While identical machines are the most utilized parallel machines in stages of HFS environment because of theoretical easiness, unrelated and uniform machines follow them respectively. Besides, it is possible to say that unrelated and uniform machines are more suitable for real-life scheduling problems.

Setup times (sequence-dependent or sequence-independent), batch processing, stage skipping, machine eligibility, and transportation times are the most utilized constraints. Despite $91 \%$ usage of job/machine related constraints, energy-related constraints such as variable speed levels, TOU electricity prices and turn on/off strategy are more considered in recent years to minimize energy consumption and to realize more environment friendly production.

It is observed that there are more single-objective HFS articles compared to multi-objective articles in the literature. At this point, it is possible to say that there is an opportunity in the multi-objective scheduling problems. Makespan, total tardiness, and total earliness are the most utilized criteria in HFSPs. At this point, we can infer that effective system utilization and customer satisfaction are mostly aimed to provide in the included articles.

As we mentioned before, 172 HFS articles which utilize a mathematical model to express the scheduling problem have been analyzed in this review paper. Although mathematical models can be solved for small-sized instances, it is required to apply heuristic or metaheuristic algorithms to obtain solutions for real-size problems in a reasonable time. MIP and $\varepsilon$-constraint methods are utilized to obtain exact solutions for single-objective and multi-objective HFSPs respectively. Full enumeration method, iterative relaxation algorithm and B\&B algorithm can be ranked as other exact algorithms. Metaheuristics are the most applied solution methods as single or hybrid to cope with HFSPs since these problems are in the NP-hard class of optimization problems. While 42 different metaheuristics have been utilized in 172 articles, GA is the most applied one. SA, PSO, VNS, ACO, and ABC follow it respectively and besides NSGA-II is the most utilized metaheuristic to solve multiobjective HFSPs. Hybrid metaheuristics combining metaheuristic algorithms with other methods (metaheuristic, heuristic or another method) has become common in recent years in order to obtain better solutions. Due to the importance of initial solutions on the final solutions, heuristics or specific rules are utilized with metaheuristics to obtain initial solutions.

Since the scheduling problems have distinctive constraints and parameters BPs could not be generally utilized for HFSPs and therefore RGTPs are the most utilized test instances. Different real-life HFSPs are located in the literature from various industries such as steel, electronic and automotive. On the other hand, despite the common usage of deterministic parameters, processing times, setup times and due dates can be more utilized as stochastic or fuzzy parameters to cope with uncertainties in the scheduling environment and to obtain more realistic results.

As a result of this systematic, extensive, and updated analysis of HFS literature, it is possible to say that due to increasing importance of cleaner production and energy-oriented scheduling concepts utilization of energy-related constraints and environment-related criteria can be beneficial for acceptance of publication. That is, multi-objective problems which handle more than one of completion time-based, due date-based, environment-related and cost-based criteria simultaneously can be more acceptable in today's competitive manufacturing conditions. Distributed scheduling has become popular in the HFS literature recently. Besides, stochastic or fuzzy parameters can be utilized in order to obtain more realistic results. New versions of metaheuristics can be suggested to obtain better solutions in terms of objective value and computational time. 
Moreover, it is obviously seen that the usage of environment-related criteria such as TEC and TECC increases day by day due to the importance of cleaner production.

\section{Appendix A}

Abbreviations of algorithms existing in Section 3.2

\begin{tabular}{cccc}
\hline Abbrevation & Expansion & Abbrevation & Expansion \\
\hline ABC & Artificial Bee Colony & ILS & Iterated Local Search \\
ACO & Ant Colony Optimization & IPG & Iterated Pareto Greedy \\
AIS & Artificial Immune System & LR & Lagrangian Relaxation \\
ALO & Ant Lion Optimization & MBO & Migrating Birds Optimization \\
B\&B & Branch and Bound Algorithm & MOEA/D & Multiobjective Evolutionary Algorithm \\
Based on Decomposition
\end{tabular}

\section{References}

Abdollahpour S., \& Rezaian J. (2017). Two new meta-heuristics for no-wait flexible flow shop scheduling problem with capacitated machines, mixed make-to-order and make-to-stock policy. Soft Computing, 21, 3147-3165.

Asadi-Gangraj E. (2018). Lagrangian relaxation approach to minimizing makespan in hybrid flow shop scheduling problem with unrelated parallel machines., Scientia Iranica, 25(6), 3765-3775.

Assadipour G., Ke G. Y., \& Verma M. (2014). An analytical framework for integrated maritime terminal scheduling problems with time windows. Expert Systems with Applications, 41, 7415-7424.

Attar S. F., Mohammadi M., \& Tavakkoli-Moghaddam R. (2013). Hybrid flexible flowshop scheduling problem with unrelated parallel machines and limited waiting times. International Journal of Advanced Manufacturing Technology, 68, $1583-1599$.

Attar S. F., Mohammadi M., Tavakkoli-Moghaddam R., \& Yaghoubi S. (2014). Solving a new multi-objective hybrid flexible flowshop problem with limited waiting times and machine-sequence-dependent set-up time constraints. International Journal of Computer Integrated Manufacturing, 27(5), 450-469.

Azami A., Demirli K., \& Bhuiyan N. (2018). Scheduling in aerospace composite manufacturing systems: a two-stage hybrid flow shop problem. International Journal of Advanced Manufacturing Technology, 95, 3259-3274.

Baxendale M., McGree J. M., Bellette A., \& Corry P. (2020). Machine-based production scheduling for rotomoulded plastics manufacturing. International Journal of Production Research, 10.1080/00207543.2020.1727046.

Behnamian J., \& Fatemi Ghomi S. M. T. (2011). Hybrid flowshop scheduling with machine and resource-dependent processing times. Applied Mathematical Modelling, 35, 1107-1123.

Behnamian J., \& Fatemi Ghomi S. M. T. (2014). Multi-objective fuzzy multiprocessor flowshop scheduling. Applied Soft Computing, 21, 139-148.

Behnamian J., \& Zandieh M. (2011). A discrete colonial competitive algorithm for hybrid flowshop scheduling to minimize earliness and quadratic tardiness penalties. Expert Systems with Applications, 38, 14490-14498.

Behnamian J., \& Zandieh M. (2013). Earliness and Tardiness Minimizing on a Realistic Hybrid Flowshop Scheduling with Learning Effect by Advanced Metaheuristic. Arabian Journal for Science and Engineering, 38, 1229-1242.

Behnamian J., Fatemi Ghomi S. M. T., \& Zandieh M. (2012). Hybrid flowshop scheduling with sequence-dependent setup times by hybridizing max-min ant system, simulated annealing and variable neighbourhood search. Expert Systems, 29(2), $156-169$. 
Behnamian J., Fatemi Ghomi S. M. T., \& Zandieh M. (2014). Realistic variant of just-in-time flowshop scheduling: integration of Lp-metric method in PSO-like algorithm. International Journal of Advanced Manufacturing Technology, 75, $1787-1797$.

Behnamian J., Fatemi Ghomi S. M. T., \& Zandieh M. (2010). Development of a hybrid metaheuristic to minimise earliness and tardiness in a hybrid flowshop with sequence-dependent setup times. International Journal of Production Research, 48(5), 14151438.

Bing-hai Z., Meng Y., \& Zhi-qiang L. (2016). An improved Lagrangian relaxation heuristic for the scheduling problem of operating theatres. Computers \& Industrial Engineering, 101, 490-503.

Boroun M., Ramezani S., Farahani N. V., Hassannayebi E., Abdolmaali S., \& Shakibayifar M. (2020). An efficient heuristic method for joint optimization of train scheduling and stop planning on double-track railway systems. INFOR: Information Systems and Operational Research, 58(4), 652-679.

Boudhar M., \& Meziani N. (2010). Two-stage hybrid flow shop with recirculation. International Transactions in Operational Research, 17, 239-255.

Bozorgirad M. A., \& Logendran R. (2016). A comparison of local search algorithms with population-based algorithms in hybrid flow shop scheduling problems with realistic characteristics. International Journal of Advanced Manufacturing Technology, 83, 1135-1151.

Bozorgnezhad F., Asadi-Gangraj E., \& Paydar M. M. (2019). A Simultaneous Worker Assignment and Scheduling Problem for Minimizing Makespan in Flexible Flow Shop Via Metaheuristic Approaches. International Journal of Industrial Engineering \& Production Research, 30, 207-223.

Cao C., Zhang Y., Gu X., Li D., \& Li J. (2020a). An improved gravitational search algorithm to the hybrid flowshop with unrelated parallel machines scheduling problem. International Journal of Production Research, 10.1080/00207543.2020.1788732.

Cao J., Pan R., Xia X., Shao X., \& Wang X. (2020b). An efficient scheduling approach for an iron-steel plant equipped with selfgeneration equipment under time-of-use electricity tariffs. Swarm and Evolutionary Computation, 60, 100764.

Chabouh S., Hammami S., Marcon E., \& Bouchriha H. (2018). Appointment scheduling of inpatients and outpatients in a multistage integrated surgical suite: Application to a Tunisian ophthalmology surgery department. Journal of Simulation, 12(1), 67-75.

Chamnanlor C., Sethanan K., Gen M., \& Chien CF. (2017). Embedding ant system in genetic algorithm for re-entrant hybrid flow shop scheduling problems with time window constraints. Journal of Intelligent Manufacturing, 28, 1915-1931.

Chen TL., Cheng CY., \& Chou YH. (2020). Multi-objective genetic algorithm for energy-efficient hybrid flow shop scheduling with lot streaming. Annals of Operations Research, 290, 813-836.

Chen YY., Cheng CY., Wang LC., \& Chen TL. (2013). A hybrid approach based on the variable neighborhood search and particle swarm optimization for parallel machine scheduling problems - A case study for solar cell industry., International Journal of Production Economics, 141, 66-78.

Cheng CY., Chen TL., Wang LC., \& Chen YY. (2013). A genetic algorithm for the multi-stage and parallel-machine scheduling problem with job splitting - A case study for the solar cell industry. International Journal of Production Research, 51(16), 47554777.

Cho HM., Bae SJ., Kim J., \& Jeong IJ. (2011). Bi-objective scheduling for reentrant hybrid flow shop using Pareto genetic algorithm. Computers \& Industrial Engineering, 61, 529-541.

Choi S. H., \& Wang K. (2012). Flexible flow shop scheduling with stochastic processing times: A decomposition-based approach. Computers \& Industrial Engineering, 63, 362-373.

Chung TP., \& Chen F. (2019). A complete immunoglobulin-based artificial immune system algorithm for two-stage assembly flowshop scheduling problem with part splitting and distinct due windows. International Journal of Production Research, 57(10), 3219-3237.

Cortes B. M., Garcia J. C. E., \& Hernandez F. R. (2012). Multi-objective flow-shop scheduling with parallel machines. International Journal of Production Research, 50(10), 2796-2808.

Costa A., Cappadonna F. \& A., Fichera S. (2014). A novel genetic algorithm for the hybrid flow shop scheduling with parallel batching and eligibility constraints. International Journal of Advanced Manufacturing Technology, 75, 833-847.

Costa A., Cappadonna F. A., \& Fichera S. (2013). A dual encoding-based meta-heuristic algorithm for solving a constrained hybrid flow shop scheduling problem. Computers \& Industrial Engineering, 64, 937-958.

Costa A., Fernandez-Viagas V., \& Framinan J. M. (2020). Solving the hybrid flow shop scheduling problem with limited human resource constraint. Computers \& Industrial Engineering, 146, 106545.

Cui H., \& Luo X. (2017). An improved Lagrangian relaxation approach to scheduling steelmaking-continuous casting process. Computers and Chemical Engineering, 106, 133-146.

Cui H., Luo X., \& Wang Y. (2020). Scheduling of steelmaking-continuous casting process using deflected surrogate Lagrangian relaxation approach and DC algorithm. Computers \& Industrial Engineering, 140, 106271.

Dai M., Tang D., Giret A., Salido M. A., \& Li W. D. (2013a). Energy-efficient scheduling for a flexible flow shop using an improved genetic-simulated annealing algorithm. Robotics and Computer-Integrated Manufacturing, 29, 418-429.

Dai M., Tang D., Zheng K., \& Cai Q. (2013b). An Improved Genetic-Simulated Annealing Algorithm Based on a Hormone Modulation Mechanism for a Flexible Flow-Shop Scheduling Problem. Advances in Mechanical Engineering, 2013, 13 pages.

Dasgupta P., \& Das S. (2015). A Discrete Inter-Species Cuckoo Search for flowshop scheduling problems. Computers \& Operations Research, 60, 111-120.

Defersha F. M. (2011). A comprehensive mathematical model for hybrid flexible flowshop lot streaming problem. International Journal of Industrial Engineering Computations, 2, 283-294. 
Defersha F. M. (2015). A simulated annealing with multiple-search paths and parallel computation for a comprehensive flowshop scheduling problem. International Transactions in Operational Research, 22(4), 669-691.

Defersha F. M., \& Chen M. (2012). Mathematical model and parallel genetic algorithm for hybrid flexible flowshop lot streaming problem. International Journal of Advanced Manufacturing Technology, 62, 249-265.

Ding J., Schulz S., Shen L., Buscher U., \& Lü Z. (2020). Energy aware scheduling in flexible flow shops with hybrid particle swarm optimization. Computers and Operations Research, 10.1016/j.cor.2020.105088.

Elmi A., \& Topaloglu S. (2013). A scheduling problem in blocking hybrid flow shop robotic cells with multiple robots. Computers \& Operations Research, 40, 2543-2555.

Elmi A., \& Topaloglu S. (2014). Scheduling multiple parts in hybrid flow shop robotic cells served by a single robot. International Journal of Computer Integrated Manufacturing, 27(12), 1144-1159.

Engin O., \& Engin B. (2018). Hybrid flow shop with multiprocessor task scheduling based on earliness and tardiness penalties. Journal of Enterprise Information Management, 31(6), 925-936.

Farahmand-Mehr M., Fattahi P., Kazemi M., Zarei H., \& Piri A. (2014). An efficient genetic algorithm for a hybrid flow shop scheduling problem with time lags and sequence-dependent setup time. Manufacturing Review, 1, 21.

Fattahi P., Hosseini S. M. H., \& Jolai F. (2013). A mathematical model and extension algorithm for assembly flexible flow shop scheduling problem. International Journal of Advanced Manufacturing Technology, 65, 787-802.

Feng H., Tan C., Xia T., Pan E., \& Xi L. (2019). Joint optimization of preventive maintenance and flexible flowshop sequencedependent group scheduling considering multiple setups. Engineering Optimization, 51(9), 1529-1546.

Feng X., Zheng F., \& Xu Y. (2016). Robust scheduling of a two-stage hybrid flow shop with uncertain interval processing times. International Journal of Production Research, 54(12), 3706-3717.

Fu Y., Li Z., Chen N., \& Qu C. (2020). A Discrete Multi-Objective Rider Optimization Algorithm for Hybrid Flowshop Scheduling Problem Considering Makespan, Noise and Dust Pollution. IEEE Access, 8, 88527-88546.

Garavito-Hernández E. A., Peña-Tibaduiza E., Perez-Figueredo L. E., \& Moratto-Chimenty E. (2019). A meta-heuristic based on the Imperialist Competitive Algorithm (ICA) for solving Hybrid Flow Shop (HFS) scheduling problem with unrelated parallel machines. Journal of Industrial and Production Engineering, 36(6), 362-370.

Geng K., Ye C., \& Liu A. L. (2020b). Research on Multi-Objective Hybrid Flow Shop Scheduling Problem With Dual Resource Constraints Using Improved Memetic Algorithm. IEEE Access, 8, 104527-104542.

Geng K., Ye C., Cao L., \& Liu L. (2019). Multi-Objective Reentrant Hybrid Flowshop Scheduling with Machines Turning on and off Control Strategy Using Improved Multi-Verse Optimizer Algorithm. Mathematical Problems in Engineering, 2019,18 pages.

Geng K., Ye C., Dai Z., \& Liu L. (2020a). Bi-Objective Re-Entrant Hybrid Flow Shop Scheduling considering Energy Consumption Cost under Time-of-Use Electricity Tariffs. Complexity, 2020, 17 pages.

Ghaleb M. A., \& Alharkan I. M. (2019). Particle Swarm Optimization Algorithms for Two-stage Hybrid Flowshop Scheduling Problem with No-wait. Universal Journal of Electrical and Electronic Engineering, 6(2), 46-60.

Ghodratnama A., Jolai F., \& Tavakkoli-Moghaddam R. (2015). Solving a new multi-objective multi-route flexible flow line problem by multi-objective particle swarm optimization and NSGA-II. Journal of Manufacturing Systems, 36, 189-202.

Gicquel C., Hege L., Minoux M., \& van Canneyt W. (2012). A discrete time exact solution approach for a complex hybrid flowshop scheduling problem with limited-wait constraints. Computers \& Operations Research, 39, 629-636.

Golneshini F. P., \& Fazlollahtabar H. (2019). Meta-heuristic algorithms for a clustering-based fuzzy bi-criteria hybrid flow shop scheduling problem. Soft Computing, 23, 12103-12122.

Gong G., Chiong R., Deng Q., Han W., Zhang L., Lin W., \& Li K. (2020). Energy-efficient flexible flow shop scheduling with worker flexibility. Expert Systems With Applications, 141, 112902.

González-Neira E. M., García-Cáceres R. G., Caballero-Villalobos J. P., Molina-Sánchez L. P., \& Montoya-Torres J. R. (2016). Stochastic flexible flow shop scheduling problem under quantitative and qualitative decision criteria. Computers \& Industrial Engineering, 101, 128-144.

Gonzalez-Neira, Montoya-Torres J. R., \& Barrera D. (2017). Flow-shop scheduling problem under uncertainties: Review and trends. International Journal of Industrial Engineering Computations, 8, 399-426.

Graham R. L., Lawler E. L., Lenstra J. K., \& Rinnooy Kan A. H. G. (1979). Optimization and Approximation in Deterministic Sequencing and Scheduling: a Survey. Annals of Discrete Mathematics, 5, 287-326.

Guan S., Shikanai T., Nakamura M., \& Fukami K. (2017). Mathematical model and solution for land-use crop planning with cooperative work. 6th IIAI International Congress on Advanced Applied Informatics, Hamamatsu, Japan, 903-908.

Guo Q., Tang L., Liu J., \& Zhao S. (2020). Continuous-time formulation and differential evolution algorithm for an integrated batching and scheduling problem in aluminium industry. International Journal of Production, 10.1080/00207543.2020.1747656.

Hachicha H. K., \& Mansour F. Z. (2018). Two-MILP models for scheduling elective surgeries within a private healthcare facility. Health Care Management Science, 21, 376-392.

Han Z., Han C., Lin S., Dong X., \& Shi H. (2019b). Flexible Flow Shop Scheduling Method with Public Buffer. Processes, 7, 681.

Han Z., Zhang Q., Shi H., \& Zhang J. (2019a). An Improved Compact Genetic Algorithm for Scheduling Problems in a Flexible Flow Shop with a Multi-Queue Buffer. Processes, 7, 302.

Hao JH., Li JQ., Du Y., Song MX., Duan P., \& Zhang YY. (2019). Solving Distributed Hybrid Flowshop Scheduling Problems by a Hybrid Brain Storm Optimization Algorithm. IEEE Access, 7, 66879-66894. 
Hasani A., \& Hosseini S. M. H. (2020). A bi-objective flexible flow shop scheduling problem with machine-dependent processing stages: Trade-offbetween production costs and energy consumption. Applied Mathematics and Computation, 386, 125533.

He X., Dong S., \& Zhao N. (2020). Research on rush order insertion rescheduling problem under hybrid flow shop based on NSGAIII. International Journal of Production Research, 58(4), 1161-1177.

Hekmatfar M., Fatemi Ghomi S. M. T., \& Karimi B. (2011). Two stage reentrant hybrid flow shop with setup times and the criterion of minimizing makespan. Applied Soft Computing, 11, 4530-4539.

Huang J. D., Liu J. J., Chen Q. X., \& Mao N. (2017). Minimizing makespan in a two-stage flow shop with parallel batch-processing machines and reentrant jobs. Engineering Optimization, 49(6), 1010-1023.

Huang R. H., Yang CL., \& Liu SC. (2015a). No-Wait Flexible Flow Shop Scheduling with Due Windows. Mathematical Problems in Engineering, 2015, 9 pages.

Huang RH., \& Yu SC. (2013). Clarifying Cutting and Sewing Processes with Due Windows Using an Effective Ant Colony Optimization. Mathematical Problems in Engineering, 2013, 12 pages.

Huang RH., \& Yu SC. (2016). Two-stage multiprocessor flow shop scheduling with deteriorating maintenance in cleaner production. Journal of Cleaner Production, 135, 276-283.

Huang RH., Yang CL., \& Hsu CT. (2015b). Multi-objective two-stage multiprocessor flow shop scheduling - a subgroup particle swarm optimisation approach. International Journal of Systems Science, 46(16), 3010-3018.

Huang RH., Yu SC., \& Kuo CW. (2014). Reentrant two-stage multiprocessor flow shop scheduling with due windows. International Journal of Advanced Manufacturing Technology, 71, 1263-1276.

Hwang F. J., \& Lin B. M. T. (2018). Survey and extensions of manufacturing models in two-stage flexible flow shops with dedicated machines. Computers \& Operations Research, 98, 103-112.

Javadian N., Fattahi P., Farahmand-Mehr M., Amiri-Aref M., \& Kazemi M. (2012). An immune algorithm for hybrid flow shop scheduling problem with time lags and sequence-dependent setup times. International Journal of Advanced Manufacturing Technology, 63, 337-348.

Jiang S., Liu M., Hao J., \& Qian W. (2015). A bi-layer optimization approach for a hybrid flow shop scheduling problem involving controllable processing times in the steelmaking industry. Computers \& Industrial Engineering, 87, 518-531.

Johnson S. M. (1954). Optimal two- and three-stage production schedules with setup times included. Naval Research Logistics Quarterly, 1(1), 61-68.

Jolai F., Asefi H., Rabiee M., \& Ramezani P. (2013). Bi-objective simulated annealing approaches for no-wait two-stage flexible flow shop scheduling problem. Scientia Iranica, 20(3), 861-872.

Jubiz-Diaz M., Santander-Mercado A., \& Candelo-Becerra J. E. (2019). A multi-item multi-packaging model to minimise cost of lost units, unpacking cost and CO2 emissions. International Journal of Production Research, 57(20), 6246-6263.

Karimi N., Zandieh M., \& Karamooz, H. R. (2010). Bi-objective group scheduling in hybrid flexible flowshop: A multi-phase approach. Expert Systems with Applications, 37, 4024-4032.

Kaya I., Colak M., \& Terzi F. (2019). A comprehensive review of fuzzy multi criteria decision making methodologies for energy policy making. Energy Strategy Reviews, 24, 207-228.

Keshavarz T., \& Salmasi N. (2013). Makespan minimisation in flexible flowshop sequence-dependent group scheduling problem. International Journal of Production Research, 51(20), 6182-6193.

Kheirandish O., Tavakkoli-Moghaddam R. \&, Karimi-Nasab M. (2015). An artificial bee colony algorithm for a two stage hybrid flowshop scheduling problem with multilevel product structures and requirement operations. International Journal of Computer Integrated Manufacturing, 28(5), 437-450.

Komaki G. M., Teymourian E., \& Kayvanfar V. (2016). Minimising makespan in the two-stage assembly hybrid flow shop scheduling problem using artificial immune systems. International Journal of Production Research, 54(4), 963-983.

Kong L., Wang L., Li F., Wang G., Fu Y., \& Liu J. (2020). A New Sustainable Scheduling Method for Hybrid Flow-Shop Subject to the Characteristics of Parallel Machines. IEEE Access, 8, 79998-80009.

Kurdi M. (2019). Ant colony system with a novel Non-DaemonActions procedure for multiprocessor task scheduling in multistage hybrid flow shop. Swarm and Evolutionary Computation, 44, 987-1002.

Lee GC., Hong J. M., \& Choi S. H. (2015). Efficient Heuristic Algorithm for Scheduling Two-Stage Hybrid Flowshop with Sequence-Dependent Setup Times. Mathematical Problems in Engineering, 2015, 10 pages.

Lei C., Zhao N., Ye S., \& Wu X. (2020). Memetic algorithm for solving flexible flow-shop scheduling problems with dynamic transport waiting times. Computers \& Industrial Engineering, 139, 105984.

Li C., Lu Z., Han X., Zhang Y., \& Wang L. (2016). Integrated scheduling of a container handling system with simultaneous loading and discharging operations. Engineering Optimization, 48(3), 397-414.

Li D., Meng X., Liang Q., \& Zhao J. (2015). A heuristic-search genetic algorithm for multi-stage hybrid flow shop scheduling with single processing machines and batch processing machines. Journal of Intelligent Manufacturing, 26, 873-890.

Li J., \& Han Y. (2020). A hybrid multi-objective artificial bee colony algorithm for flexible task scheduling problems in cloud computing system. Cluster Computing, 23, 2483-2499.

Li J., Pan Q., \& Mao K. (2014a)., Suganthan P. N., Solving the steelmaking casting problem using an effective fruit fly optimisation algorithm. Knowledge-Based Systems, 72, 28-36.

Li J., Pan Q., \& Mao K. (2014b). Hybrid Particle Swarm Optimization for Hybrid Flowshop Scheduling Problem with Maintenance Activities. The Scientific World Journal, 2014, 11 pages. 
Li J., Tao X., Jia B., Han Y., Liu C., Duan P., Zheng Z., \& Sang H. (2020b). Efficient multi-objective algorithm for the lot-streaming hybrid flowshop with variable sub-lots. Swarm and Evolutionary Computation, 52, 100600.

Li L., Huo J., \& Tang O. (2011). A hybrid flowshop scheduling problem for a cold treating process in seamless steel tube production. International Journal of Production Research, 49(15), 4679-4700.

Li Y., Li X., Gao L., \& Meng L. (2020c). An improved artificial bee colony algorithm for distributed heterogeneous hybrid flowshop scheduling problem with sequence-dependent setup times. Computers \& Industrial Engineering, 147, 106638.

Li Y., Li X., Gao L., Zhang B., Pan QK., Tasgetiren M. F., \& Meng L. (2020a). A discrete artificial bee colony algorithm for distributed hybrid flowshop scheduling problem with sequence-dependent setup times. International Journal of Production Research, 10.1080/00207543.2020.1753897.

Li Y., Wang H., Li Y., \& Li L. (2019a). Patient assignment scheduling in a cloud healthcare system based on petri net and greedy based heuristic. Enterprise Information Systems, 13(4), 515-533.

Li Z., Chen Q., \& Mao N. (2013a). A heuristic algorithm for two-stage flexible flow shop scheduling with head group constraint. International Journal of Production Research, 51(3), 751-771.

Li Z., Chen Q., Mao N., Wang X., \& Liu J. (2013b). Scheduling rules for two-stage flexible flow shop scheduling problem subject to tail group constraint. International Journal of Production Economics, 146, 667-678.

Li Z., Zhong R. Y., Barenji A. V., Liu J. J., Yu C. X., \& Huang G. Q. (2019b). Bi-objective hybrid flow shop scheduling with common due date. Operational Research, 10.1007/s12351-019-00470-8.

Liao CJ., Tjandradjaja E., \& Chung TP. (2012). An approach using particle swarm optimization and bottleneck heuristic to solve hybrid flow shop scheduling problem. Applied Soft Computing, 12, 1755-1764.

Lin D., Lee C. K. M., \& Wu Z. (2012). Integrating analytical hierarchy process to genetic algorithm for re-entrant flow shop scheduling problem. International Journal of Production Research, 50(7), 1813-1824.

Lin SW., Ying KC., \& Huang CY. (2013). Multiprocessor task scheduling in multistage hybrid flowshops: A hybrid artificial bee colony algorithm with bi-directional planning. Computers \& Operations Research, 40, 1186-1195.

Liu J., Han W., Li J., Zhang Y., \& Su X. (2020a). Integration Design of Sortie Scheduling for Carrier Aircrafts Based on Hybrid Flexible Flowshop. IEEE Systems Journal, 14(1), 1503-1511.

Liu M., Yang X., Chu F., Zhang J., \& Chu C. (2020c). Energy-oriented bi-objective optimization for the tempered glass scheduling. Omega, 90, 101995.

Liu M., Yang X., Zhang J., \& Chu C. (2017). Scheduling a tempered glass manufacturing system: a three-stage hybrid flow shop model. International Journal of Production Research, 55(20), 6084-6107.

Liu Z., Yan J., Cheng Q., Yang C., Sun S., \& Xue D. (2020b). The mixed production mode considering continuous and intermittent processing for an energy-efficient hybrid flow shop scheduling. Journal of Cleaner Production, 246, 119071.

Long J., Zheng Z., Gao X., \& Pardalos PM. (2018). Scheduling a realistic hybrid flow shop with stage skipping andadjustable processing time in steel plants. Applied Soft Computing, 64, 536-549.

Lu C., Gao L., Pan Q., Li X., \& Zheng J. (2019). A multi-objective cellular grey wolf optimizer for hybrid flowshop scheduling problem considering noise pollution. Applied Soft Computing, 75, 728-749.

Luo H., Huang G. Q., Zhang Y. F., \& Dai Q. Y. (2011). Hybrid flowshop scheduling with batch-discrete processors and machine maintenance in time windows. International Journal of Production Research, 49(6), 1575-1603.

Luo J., Fujimura S., El Baz D., \& Plazoles B. (2019). GPU based parallel genetic algorithm for solving an energy efficient dynamic flexible flow shop scheduling problem. Journal of Parallel and Distributed Computing, 133, 244-257.

Mao K., Pan Q., Pang X., \& Chai T. (2014). A novel Lagrangian relaxation approach for a hybrid flowshop scheduling problem in the steelmaking-continuous casting process. European Journal of Operational Research, 236, 51-60.

Marichelvam M. K., Geetha M., \& Tosun O. (2020). An improved particle swarm optimization algorithm to solve hybrid flowshop scheduling problems with the effect of human factors - A case study. Computers and Operations Research, 114, 104812.

Marichelvam M. K., Prabaharan T., \& Yang X. S. (2014a). A Discrete Firefly Algorithm for the Multi-Objective Hybrid Flowshop Scheduling Problems. IEEE Transactions on Evolutionary Computation, 18(2), 301-305.

Marichelvam M. K., Prabaharan T., \& Yang X. S. (2014b). Improved cuckoo search algorithm for hybrid flow shop scheduling problems to minimize makespan. Applied Soft Computing, 19, 93-101.

Meghdari A., Fazlollahtabar S., \& Darband H. (2015). Flexible Flow Shop Scheduling with Learning and Forgetting Effects. Journal of Mechanical Engineering Research and Developments, 38(2), 116-125.

Meng L., Zhang C., Shao X., Ren Y., \& Ren C. (2019). Mathematical modelling and optimisation of energy-conscious hybrid flow shop scheduling problem with unrelated parallel machines. International Journal of Production Research, 57(4), 1119-1145.

Moher D., Liberati A., Tetzlaff J., \& Altman D. G. (2009a). Preferred Reporting Items for Systematic Reviews and Meta-Analyses: The PRISMA Statement. Annals of Internal Medicine, 151(4), 264-270.

Moher D., Liberati A., Tetzlaff J., \& Altman D. G. (2009b). Preferred Reporting Items for Systematic Reviews and Meta-Analyses: The PRISMA Statement, Plos Medicine, 6(7), 1-6.

Mollaei A., Mohammadi M., \& Naderi B. (2019). A bi-objective MILP model for blocking hybrid flexible flow shop scheduling problem: robust possibilistic programming approach. International Journal of Management Science and Engineering Management, 14(2), 137-146.

Morais M. F., Filho M. G., \& Boiko T. J. P. (2013). Hybrid Flow Shop Scheduling Problems Involving Setup Considerations: A Literature Review and Analysis. International Journal of Industrial Engineering, 20(11-12), 614-630. 
Naderi B., \& Yazdani M. (2014). A model and imperialist competitive algorithm for hybrid flow shops with sublots and setup times. Journal of Manufacturing Systems, 33, 647-653.

Naderi B., Gohari S., \& Yazdani M. (2014). Hybrid flexible flowshop problems: Models and solution methods. Applied Mathematical Modelling, 38, 5767-5780.

Nejati M., Mahdavi I., Hassanzadeh R., \& Mahdavi-Amiri N. (2016). Lot streaming in a two-stage assembly hybrid flow shop scheduling problem with a work shift constraint. Journal of Industrial and Production Engineering, 33(7), 459-471.

Nejati M., Mahdavi I., Hassanzadeh R., Mahdavi-Amiri N., \& Mojarad M. S. (2014). Multi-job lot streaming to minimize the weighted completion time in a hybrid flow shop scheduling problem with work shift constraint. International Journal of Advanced Manufacturing Technology, 70, 501-514.

Neufeld J. S., Gupta J. N. D., \& Buscher U. (2016). A comprehensive review of flowshop group scheduling literature. Computers \& Operations Research, 70, 56-74.

Nikzad F., Rezaeian J., Mahdavi I., \& Rastgar I. (2015). Scheduling of multi-component products in a two-stage flexible flow shop. Applied Soft Computing, 32, 132-143.

Nishi T., Hiranaka Y., \& Inuiguchi M. (2010). Lagrangian relaxation with cut generation for hybrid flowshop scheduling problems to minimize the total weighted tardiness. Computers \& Operations Research, 37, 189-198.

Niu Q., Zhou T., Fei M., \& Wang B. (2012). An efficient quantum immune algorithm to minimize mean flow time for hybrid flow shop problems. Mathematics and Computers in Simulation, 84, 1-25.

Oztop H., Tasgetiren M. F., Kandiller L., Türsel Eliiyi D., \& Gao L. (2020). Ensemble of metaheuristics for energy-efficient hybrid flowshops: Makespan versus total energy consumption. Swarm and Evolutionary Computation, 54, 100660.

Oztop H., Tasgetiren M. F., Türsel Eliiyi D., \& Pan QK. (2019). Metaheuristic algorithms for the hybrid flowshop scheduling problem. Computers and Operations Research, 111, 177-196.

Pan QK. (2016). An effective co-evolutionary artificial bee colony algorithm for steelmaking-continuous casting scheduling. European Journal of Operational Research, 250, 702-714.

Pan QK., \& Dong Y. (2014). An improved migrating birds optimisation for a hybrid flowshop scheduling with total flowtime minimisation. Information Sciences, 277, 643-655.

Pan QK., Wang L., Li JQ., \& Duan JH. (2014). A novel discrete artificial bee colony algorithm for the hybrid flowshop scheduling problem with makespan minimisation. OMEGA, 45, 42-56.

Pargar F., \& Zandieh M. (2012). Bi-criteria SDST hybrid flow shop scheduling with learning effect of setup times: water flow-like algorithm approach. International Journal of Production Research, 50(10), 2609-2623.

Peng K., Pan Q., \& Zhang B. (2018b). An improved artificial bee colony algorithm for steelmaking-refining-continuous casting scheduling problem. Chinese Journal of Chemical Engineering, 26, 1727-1735.

Peng K., Pan QK., Gao L., Zhang B., \& Pang X. (2018a). An Improved Artificial Bee Colony algorithm for real-world hybrid flowshop rescheduling in Steelmaking-Refining-Continuous Casting process. Computers \& Industrial Engineering, 122, 235250.

Pinedo M. L. (2008). Scheduling theory, algorithms, and systems. 3rd ed., Springer, New York.

Qin T., Du Y., Chen J. H., \& Sha M. (2020). Combining mixed integer programming and constraint programming to solve the integrated scheduling problem of container handling operations of a single vessel. European Journal of Operational Research, 285(3), 884-901.

Qin W., Zhuang Z., Liu Y., \& Tang O. (2019). A two-stage ant colony algorithm for hybrid flow shop scheduling with lot sizing and calendar constraints in printed circuit board assembly. Computers \& Industrial Engineering, 138, 106-115.

Rahmani D., \& Ramezanian R. (2016). A stable reactive approach in dynamic flexible flow shop scheduling with unexpected disruptions: A case study. Computers \& Industrial Engineering, 98, 360-372.

Rahmani D., Heydari M., Makui A., \& Zandieh M. (2013). A new approach to reducing the effects of stochastic disruptions in flexible flow shop problems with stability and nervousness. International Journal of Management Science and Engineering Management, 8(3), 173-178.

Raissi S., Rooeinfar R., \& Ghezavati V. R. (2019). Three Hybrid Metaheuristic Algorithms for Stochastic Flexible Flow Shop Scheduling Problem with Preventive Maintenance and Budget Constraint. Journal of Optimization in Industrial Engineering, 12(2), 131-147.

Ramezanian R., Sanami S. F., \& Nikabadi M .S. (2017). A simultaneous planning of production and scheduling operations in flexible flow shops: case study of tile industry. International Journal of Advanced Manufacturing Technology, 88, $2389-2403$.

Ribas I., Leisten R., \& Framinan J. M. (2010). Review and classification of hybrid flowshop scheduling problems from a production system and a solutions procedure perspective. Computers \& Operations Research, 37, 1439-1454.

Rooeinfar R., Raissi S., \& Ghezavati VR. (2019). Stochastic flexible flow shop scheduling problem with limited buffers and fixed interval preventive maintenance: a hybrid approach of simulation and metaheuristic algorithms. Simulation, 95(6), 509-528.

Rossi A., Puppato A., \& Lanzetta M. (2013). Heuristics for scheduling a two-stage hybrid flow shop with parallel batching machines: application at a hospital sterilisation plant. International Journal of Production Research, 51(8), 2363-2376.

Ruiz R., \& Vazquez-Rodriguez J. A. (2010). The hybrid flow shop scheduling problem. European Journal of Operational Research, $205,1-18$.

Sawik T. (2012). Batch versus cyclic scheduling of flexible flow shops by mixed-integer programming. International Journal of Production Research, 50(18), 5017-5034. 
Schulz S., Buscher U., \& Shen L. (2020). Multi-objective hybrid flow shop scheduling with variable discrete production speed levels and time-of-use energy prices. Journal of Business Economics, 90, 1315-1343.

Schulz S., Neufeld J. S., \& Buscher U. (2019). A multi-objective iterated local search algorithm for comprehensive energy-aware hybrid flow shop scheduling. Journal of Cleaner Production, 224, 421-434.

Seidgar H., Zandieh M., \& Mahdavi I. (2016). Bi-objective optimization for integrating production and preventive maintenance scheduling in two-stage assembly flow shop problem. Journal of Industrial and Production Engineering, 33(6), 404-425.

Shahvari O., Salmasi N., Logendran R., \& Abbasi B. (2012). An efficient tabu search algorithm for flexible flow shop sequencedependent group scheduling problems. International Journal of Production Research, 50(15), 4237-4254.

Shao W., Shao Z., \& Pi D. (2020). Modeling and multi-neighborhood iterated greedy algorithm for distributed hybrid flow shop scheduling problem. Knowledge-Based Systems, 194, 105527.

Sheikh S. (2013). Multi-objective flexible flow lines with due window, time lag, and job rejection. International Journal of Advanced Manufacturing Technology, 64, 1423-1433.

Shen J., Wang L., \& Zheng H. (2016). A modified teaching-learning-based optimisation algorithm for bi-objective re-entrant hybrid flowshop scheduling. International Journal of Production Research, 54(12), 3622-3639.

Shiau DF., \& Huang YM. (2012). A hybrid two-phase encoding particle swarm optimization for total weighted completion time minimization in proportionate flexible flow shop scheduling. International Journal of Advanced Manufacturing Technology, 58, 339-357.

Shim SO., Park KB., \& Choi SY. (2017). Innovative Production Scheduling with Customer Satisfaction Based Measurement for the Sustainability of Manufacturing Firms. Sustainability, 9, 2249.

Solano-Charris E. L., Montoya-Torres J. R., \& Paternina-Arboleda C. D. (2011). Ant colony optimization algorithm for a Bi-criteria 2-stage hybrid flowshop scheduling problem. Journal of Intelligent Manufacturing, 22, 815-822.

Soltani S. \& A., Karimi B. (2015). Cyclic hybrid flow shop scheduling problem with limited buffers and machine eligibility constraints. International Journal of Advanced Manufacturing Technology, 76, 1739-1755.

Sun Y., \& Qi X. (2020). A DE-LS Metaheuristic Algorithm for Hybrid Flow-Shop Scheduling Problem considering Multiple Requirements of Customers. Scientific Programming, 2020, 8811391.

Tadayon B., \& Salmasi N. (2013). A two-criteria objective function flexible flowshop scheduling problem with machine eligibility constraint. International Journal of Advanced Manufacturing Technology, 64, 1001-1015.

Tan Y., Mönch L., \& Fowler J. W. (2018). A hybrid scheduling approach for a two-stage flexible flow shop with batch processing machines. Journal of Scheduling, 21, 209-226.

Tang D., Dai M., Salido M. A., \& Giret A. (2016). Energy-efficient dynamic scheduling for a flexible flow shop using an improved particle swarm optimization. Computers in Industry, 81, 82-95.

Tang J., \& Song J. (2010). Discrete particle swarm optimisation combined with no-wait algorithm in stages for scheduling mill roller annealing process. International Journal of Computer Integrated Manufacturing, 23(11), 979-991.

Wang B., Huang K., \& Li T. (2018). Two-stage hybrid flowshop scheduling with simultaneous processing machines. Journal of Scheduling, 21, 387-411.

Wang K., \& Choi S. H. (2012). A decomposition-based approach to flexible flow shop scheduling under machine breakdown. International Journal of Production Research, 50(1), 215-234.

Wang K., \& Choi S. H. (2014). A holonic approach to flexible flow shop scheduling under stochastic processing times. Computers \& Operations Research, 43, 157-168.

Wang K., Choi S. H., \& Qin H. (2014b). An estimation of distribution algorithm for hybrid flow shop scheduling under stochastic processing times. International Journal of Production Research, 52(24), 7360-7376.

Wang LC., Chen YY., Chen TL., Cheng CY., \& Chang CW. (2014a). A hybrid flowshop scheduling model considering dedicated machines and lot-splitting for the solar cell industry. International Journal of Systems Science, 45(10), 2055-2071.

Wang S., Liu M., \& Chu C. (2015). A branch-and-bound algorithm for two-stage nowait hybrid flow-shop scheduling. International Journal of Production Research, 53(4), 1143-1167.

Wang S., Wang L., Liu M., \& Xu Y. (2013). An enhanced estimation of distribution algorithm for solving hybrid flow-shop scheduling problem with identical parallel machines. International Journal of Advanced Manufacturing Technology, 68, 20432056.

Wang S., Wang X., Chu F., \& Yu J. (2020). An energy-efficient two-stage hybrid flow shop scheduling problem in a glass production. International Journal of Production Research, 58(8), 2283-2314.

Worasan K., Sethanan K., Pitakaso R., Moonsri K., \& Nitisiri K. (2020). Hybrid particle swarm optimization and neighborhood strategy search for scheduling machines and equipment and routing of tractors in sugarcane field preparation. Computers and Electronics in Agriculture, 178, 105733.

Wu X., Shen X., \& Cui Q. (2018). Multi-Objective Flexible Flow Shop Scheduling Problem Considering Variable Processing Time due to Renewable Energy. Sustainability, 10, 841.

Xiong F., Xing K., \& Wang F. (2015). Scheduling a hybrid assembly-differentiation flowshop to minimize total flow time. European Journal of Operational Research, 240, 338-354.

Xu Y., Wang L., Wang S., \& Liu M. (2013). An effective shuffled frog-leaping algorithm for solving the hybrid flow-shop scheduling problem with identical parallel machines. Engineering Optimization, 45(12), 1409-1430. 
Xuan H., Zhang H., \& Li B. (2019). An Improved Discrete Artificial Bee Colony Algorithm for Flexible Flowshop Scheduling with Step Deteriorating Jobs and Sequence-Dependent Setup Times. Mathematical Problems in Engineering, 2019 , 13 pages.

Yalaoui N., Ouazene Y. ,Yalaoui F., Amodeo L., \& Mahdi H. (2013). Fuzzy-metaheuristic methods to solve a hybrid flow shop scheduling problem with preassignment. International Journal of Production Research, 51(12), 3609-3624.

Yan J., Li L., Zhao F., Zhang F., \& Zhao Q. (2016). A multi-level optimization approach for energy-efficient flexible flow shop scheduling. Journal of Cleaner Production, 137, 1543-1552.

Ying KC., \& Lin SW. (2018). Minimizing makespan for the distributed hybrid flowshop scheduling problem with multiprocessor tasks. Expert Systems With Applications, 92, 132-141.

Yu JM., Huang R., \& Lee DH. (2017). Iterative algorithms for batching and scheduling to minimise the total job tardiness in twostage hybrid flow shops. International Journal of Production Research, 55(11), 3266-3282.

$\mathrm{Yu}$ SC. (2014). Elucidating multiprocessors flow shop scheduling with dependent setup times using a twin particle swarm optimization. Applied Soft Computing, 21, 578-589.

Zabihzadeh S. S., \& Rezaeian J. (2016). Two meta-heuristic algorithms for flexible flow shop schedulingproblem with robotic transportation and release time. Applied Soft Computing, 40, 319-330.

Zeng Z., Hong M., Man Y., Li J., Zhang Y., \& Liu H. (2018). Multi-object optimization of flexible flow shop scheduling with batch process - Consideration total electricity consumption and material wastage. Journal of Cleaner Production, 183, 925-939.

Zhang B., Pan Q., Gao L., Li X., Meng L., \& Peng K. (2019). A multiobjective evolutionary algorithm based on decomposition for hybrid flowshop green scheduling problem. Computers \& Industrial Engineering, 136, 325-344.

Zhang B., Pan Q., Gao L., Zhang X., \& Sang H., Li J. (2017). An effective modified migrating birds optimization for hybrid flowshop scheduling problem with lot streaming. Applied Soft Computing, 52, 14-27.

Zhang X. Y., \& Chen L. (2018). A re-entrant hybrid flow shop scheduling problem with machine eligibility constraints. International Journal of Production Research, 56(16), 5293-5305.

Zhou B., Hu L., \& Zhong Z. (2018). A hybrid differential evolution algorithm with estimation of distribution algorithm for reentrant hybrid flow shop scheduling problem. Neural Computing and Applications, 30, 193-209.

Zhou B., Liao X., \& Wang K. (2019). Kalman filter and multi-stage learning-based hybrid differential evolution algorithm with particle swarm for a two-stage flow shops scheduling problem. Soft Computing, 23, 13067-13083.

Ziaeifar A., Tavakkoli-Moghaddam R., \& Pichka K. (2012). Solving a new mathematical model for a hybrid flow shop scheduling problem with a processor assignment by a genetic algorithm. International Journal of Advanced Manufacturing Technology, 61, 339-349.

Zohali H., Naderi B., \& Mohammadi M. (2019b). The economic lot scheduling problem in limited-buffer flexible flow shops: Mathematical models and a discrete fruit fly algorithm. Applied Soft Computing, 80, 904-919.

Zohali H., Naderi B., Mohammadi M., \& Roshanaei V. (2019a). Reformulation, linearization, and a hybrid iterated local search algorithm for economic lot-sizing and sequencing in hybrid flow shop problems. Computers and Operations Research, 104, $127-138$.

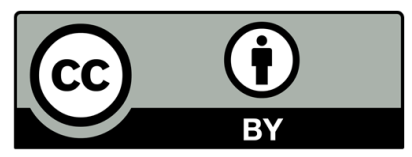

(C) 2022 by the authors; licensee Growing Science, Canada. This is an open access article distributed under the terms and conditions of the Creative Commons Attribution (CCBY) license (http://creativecommons.org/licenses/by/4.0/). 